\title{
Les galets perforés de Praileaitz I (Deba, Gipuzkoa)
}

\author{
Praileaitz leko (Deba, Gipuzkoa) errekarri zulatuak \\ The perforated pebbles from Praileaitz I (Deba, Gipuzkoa) \\ Cantos rodados perforados de Praileaitz I (Deba, Gipuzkoa)
}

MOTS-CLÉ: Magdalénien, Microscopie, Analyse d'image, Composition chimique élémentaire.

GAKO-HITZAK: Madeleine, Mikroskopia, Irudien analisia, Oinarrizko konposizio kimikoa.

KEYWORDS: Magdalenian, Microscopy, Image analysis, Elemental analysis.

PALABRAS CLAVE: Magdaleniense, Microscopía, Análisis de imágenes, Composición química elemental.

\section{Francesco D'ERRICO(1,2), Marian VANHAEREN ${ }^{(1)}$, Alain QUEFFELEC ${ }^{(1)}$}

\section{RÉSUMÉ}

Vingt-huit galets dont vingt-et-un perforés intentionnellement ont été retrouvés dans le niveau Magdalénien ancien du site de Praileaitz I situé dans la vallée du Deba. Parmi eux, quatorze ont été retrouvés alignés et très proches les uns des autres. Les analyses morphologique et chimique, par pXRF, des objets archéologiques et d'un référentiel naturel régional montrent que les galets ne proviennent pas de la vallée du Deba mais qu'ils semblent tout de même avoir une origine unique ou avoir été l'objet d'une forte sélection. L'analyse technologique montre une préparation complexe de la surface avant la perforation puis une utilisation comme pendeloques. Les pendeloques sont décorées d'entailles latérales ou de motifs composés de traits fins, gravés sur les faces plates. L'ensemble de l'analyse suggère une perte ou un dépôt en multiples phases pour la plupart des objets, au contraire des quatorze pendeloques retrouvées ensemble qui pourraient provenir d'un vêtement décoré comme une cape.

\section{LABURPENA}

Hogeita zortzi errekarri, zeinetako hogeita bat nahita zulatuta dauden, Deba haranean kokatzen den Praileaitz I aztarnategiko Behe Madeleine aldiko mailan aurkitu ziren. Haien artean, hamalau lerrokatuta eta euren artean oso hurbil aurkitu ziren. Objektu arkeologiko eta eskualdeko erreferentzia-laginen analisi morfologiko eta kimikoak, azken hau pXRF-z egina, Deba haranetik ez datozela erakusten dute, baina bai jatorri bakar batetik eta aukeraketa sendo baten ondoren. Analisi teknologikoak azaleraren prestaketa konplexu bat erakusten du zulatu aurrez, eta gero zintzilikario bezala erabili izana. Zintzilikarioak alboko ebaketez edo aurpegi lauetan grabatutako lerro finez apainduta daude. Analisi orokorrak iradokitzen du zintzilikarioen gehiengoak galera edo une ezberdinetan eratutako metaketa bat eratzen duela, multzoa osatzen zuten hamalau zintzilikarioak ordez, apaindutako jantzi batetik etor zitezkeen, adibidez kapa batetik.

\section{ABSTRACT}

Twenty-eight exogenous pebbles were recovered from the Lower Magdalenian levels of Praileaitz I, a cave located in the Deba valley. Twenty-one bear human made perforations at one end to transform them in personal ornaments, five display natural perforations, and two show no perforations. Six of the pebbles were discovered in the cave's first chamber, close to the East wall, and the remainder in the second chamber. Among the latter, fourteen were found aligned and close to each other along the East wall. Image and pXRF analyses of the archaeological objects, and natural pebbles collected at eight spots along the Deba, Urola and Bidasoa river valleys show that pebbles used as pendants were not collected in the Deba valley. Eighteen pendants were decorated with sets of notches on their edges or various patterns engraved on their flat faces. Microscopic analysis of the perforations indicates that four techniques (abrasion, single or multiple incisions, pecking, scraping) were used to prepare the pebble surface before perforation by hand rotation with flint borers and burins. Since experimental perforation by hand rotation of a pebble made of a similar raw material required five hours, and the use of nine constantly resharpened borers and burins, it is calculated that ca. 100 hours were necessary to produce the holes present on the Praileaitz I pendants. All the perforations bear use-wear traces demonstrating that the objects were used as pendants before being lost or disposed. Microscopic striations, absent on pebbles with no perforations, suggest a contact with a soft material during their use. Contextual information and differences in technology, morphology, and size between the fourteen pendants found together and the remainder of the collection suggest that the former were originally decorating a cloth, possibly a cape, lost or discarded during a single episode, while the latter correspond to objects lost or discarded by a number of people over a relatively long timespan.

\section{RESUMEN}

Veintiocho cantos rodados, de los cuales veintiuno están intencionadamente perforados fueron encontrados en el nivel del Magdaleniense inferior del yacimiento de Praileaitz I, situado en el valle del Deba. Entre ellos, se encontraron catorce alineados y muy cerca uno del

(1) Univ. Bordeaux, CNRS-UMR 5199 PACEA, B18 Allée Geoffroy St Hilaire, F-33615 Pessac, France.

(2) Institute for Archaeology, History, Cultural and Religious Studies, University of Bergen, Bergen, Norvège, francesco.derrico@u-bordeaux.fr 
otro. Los análisis morfológicos y químicos, mediante pXRF, de los objetos arqueológicos y de muestras de referencia regionales muestran que los cantos no provienen del valle del Deba, pero parecen tener un origen único y que han sido objeto de una selección fuerte. El análisis tecnológico muestra una preparación compleja de la superficie antes de la perforación y luego su uso como colgantes. Los colgantes están decorados con cortes laterales o de motivos formados por líneas finas grabadas en las caras planas. El análisis general sugiere una pérdida o un depósito en distintas fases para la mayoría de los objetos, en lugar de los catorce colgantes que se encontraban formando un conjunto que podría proceder de una prenda decorada, como una capa.

\section{1.- INTRODUCTION}

Les sites paléolithiques européens qui ont livré de riches collections d'objets de parure sont nombreux (cf. p.ex. TABORIN, 1993, 2004, STINER, 1999, ÁLVAREZ FERNANDEZ, 2006, CONARD, 2003, VANHAEREN, 2010). Rares sont cependant ceux qui ont fait l'objet de fouilles exhaustives et dans lesquelles la provenance stratigraphique et spatiale des objets est parfaitement connue. Au sein de ce lot, Praileaitz I est à notre connaissance le seul site d'habitat où les objets de parure, et en particulier les galets transformés en pendentifs, constituent la partie prépondérante du mobilier archéologique (PEÑALVER IRIBARREN, MUJIKA ALUSTIZA, 2003, PEÑALVER et al., 2017, PEÑALVER IRIBARREN, SAN JOSE SANTAMARTA, 2008). Cela pose la question des raisons pouvant expliquer la présence d'un grand nombre d'objets de cette nature dans cette petite cavité et, plus généralement, celle de la fonction du site au cours de la période d'abandon de ces objets. Même si cela peut paraître paradoxal, peu d'études se sont intéressées aux raisons qui expliquent la présence de parures dans des sites préhistoriques et les implications de ces découvertes pour l'interprétation des sites. Au Paléolithique supérieur, des parures sont associées à des sépultures sans que l'on sache, dans la plupart des cas, s'il s'agit d'ornements portés par les individus inhumés au cours de leur vie ou de parures exclusivement funéraires (VANHAEREN ET D'ERRICO, 2003a). II est sous-entendu que les parures découvertes dans des sites d'habitat doivent être interprétées comme le résultat de pertes occasionnelles d'objets isolés. Si cela était le cas, le nombre d'ornements perdus devrait être, pour une population donnée, proportionnel à la densité des vestiges liés aux activités de subsistance, reflet de la durée d'occupation du site. Pourtant rares sont les études qui comptabilisent et discutent cette relation (VANHAEREN, 2006). D'autres études interprètent la plus grande diversité en types d'objets symboliques que l'on observe dans certains sites comme la conséquence de l'utilisation du site comme lieu de rassemblement saisonnier, par opposition à des sites d'habitat plus pauvres en objets symboliques, interprétés comme des lieux fréquentés par des groupes restreints (CONKEY, 1980, DAVIDSON, 1997). Des lots d'objets de parure découverts groupés dans des sites d'habitat ont souvent été interprétés comme des cachettes ou des réserves (GROENEN, 1996). Quand cette découverte se produit dans des sites avec peu de mobilier archéologique, comme c'est le cas des grottes ornées, on a plutôt interprété ces associations comme des dépôts rituels (offrandes, ex-voto etc.). L'identification de préformes, d'objets cassés au cours du façonnage, de rejets de fabrication et de matière première brute est vue comme le témoin d'un atelier de fabrication (WHITE, 1992, CONARD, 2003, GURIOLI, 2002, VANHAEREN, 2006).

Les galets perforés de Praileaitz I ne se conforment, à première vue, à aucun de ces cas de figure. L'objectif de cette étude est de proposer une interprétation de cet ensemble s'appuyant à la fois sur le contexte archéologique et sur une analyse détaillée des vestiges. Cette dernière analyse prendra en compte les informations permettant de suivre les choix effectués par les préhistoriques qui ont utilisé les objets de parure depuis les choix des supports jusqu'à leur abandon dans la cavité.

\section{2.- CONTEXTE ARCHÉOLOGIQUE}

La fouille de la grotte de Praileaitz I a permis la découverte de vingt-huit galets de couleur noir et marron foncé (Figs. 1-7, Tabl. 1). Vingt-et-un portent des perforations anthropiques, cinq des perforations naturelles, deux ne sont pas perforés (cf. PEÑALVER, MUJIKA, 2003, PEÑALVER et al., 2017, PEÑALVER, SAN JOSE SANTAMARTA, 2008). Ces objets proviennent de plusieurs décapages corrélés à une même profondeur, considéré comme contemporains et rattachés au Magdalénien inférieur cantabrique (PEÑALVER, MUJIKA, 2003, PEÑALVER et al., 2017). Le tableau 1 présente les données contextuelles détaillées pour chaque pièce. Six galets ont été découverts dans le vestibule, près de la paroi est (Fig. 8). Un de ces galets est fracturé à sa moitié (n. 9). Un autre, aussi fracturé (n. 3), remonte avec un fragment découvert à $13 \mathrm{~m}$ de distance, près de la paroi ouest de la première salle (Fig. 8). C'est à l'intérieur de cette dernière qu'a été trouvé le reste des galets perforées. Un lot de quatorze spécimens, appelé le 'collier', se trouvait dans une zone d'un mètre carré près de la paroi est (Fig. 8). À une exception près, ces objets gisaient tous à plats et étaient disposés le long d'une ligne orientée sud-est/ nord-ouest. Dans un cas deux galets ont été découverts l'un sur l'autre avec leurs perforations superposées. Un deuxième lot, composé de deux spécimens, a été mis au jour au centre de la première salle (Fig. 8). À proximité de ce lot gisait un fragment qui remonte avec un galet trouvé un mètre plus loin (Fig. 8). Les trois derniers galets ont été exhumés le long de la paroi ouest (Fig. 8).

La proximité spatiale et la disposition des galets appartenant au 'collier' évoquent un seul événement et une contemporanéité stricte pour ce lot. Cela est pro- 


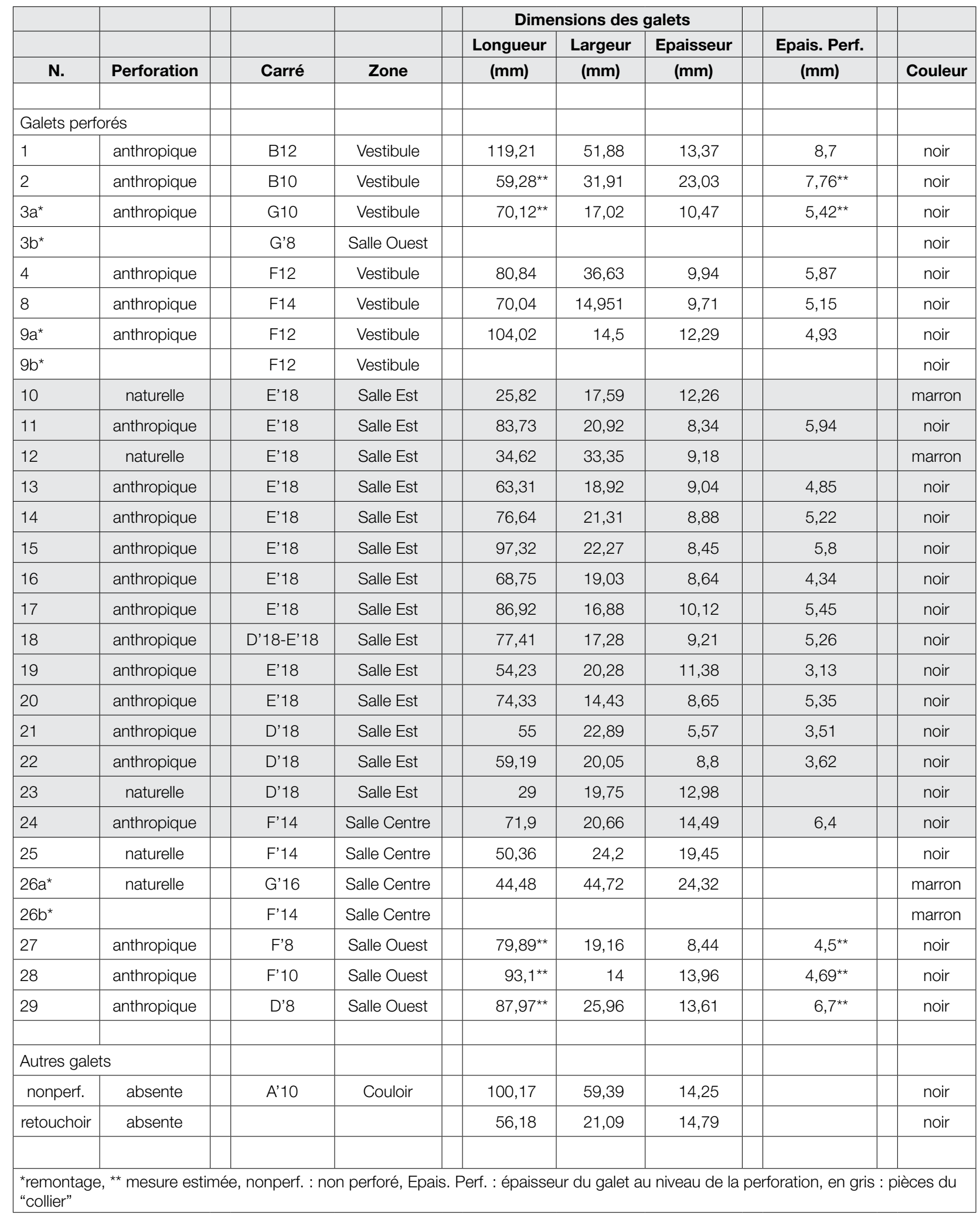

Tabl. 1. Informations contextuelles, dimensions et couleur des galets découverts à Praileaitz I. / Contextual information, dimensions and colour of the pebbles from Praileaitz I. 
LES GALETS PERFORES DE PRAILEAITZ I

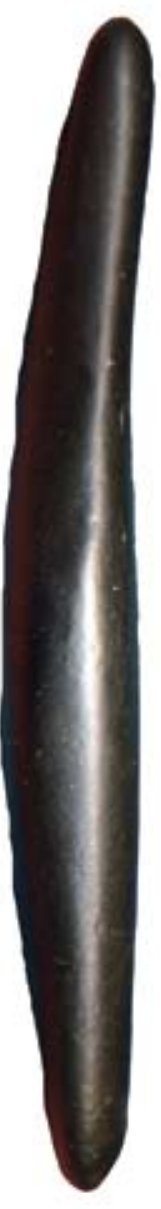

d

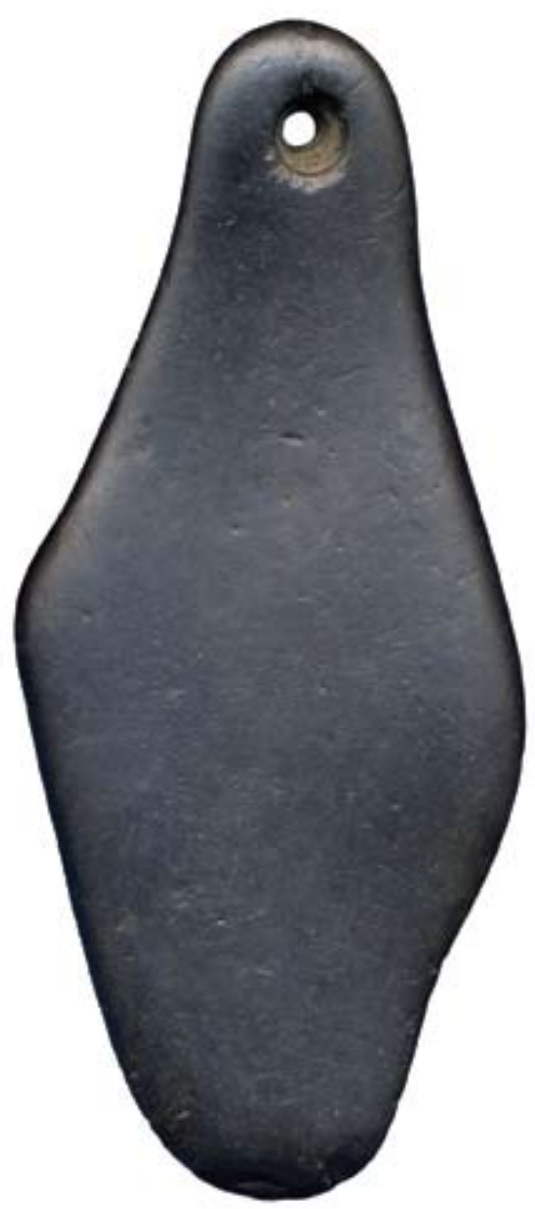

a

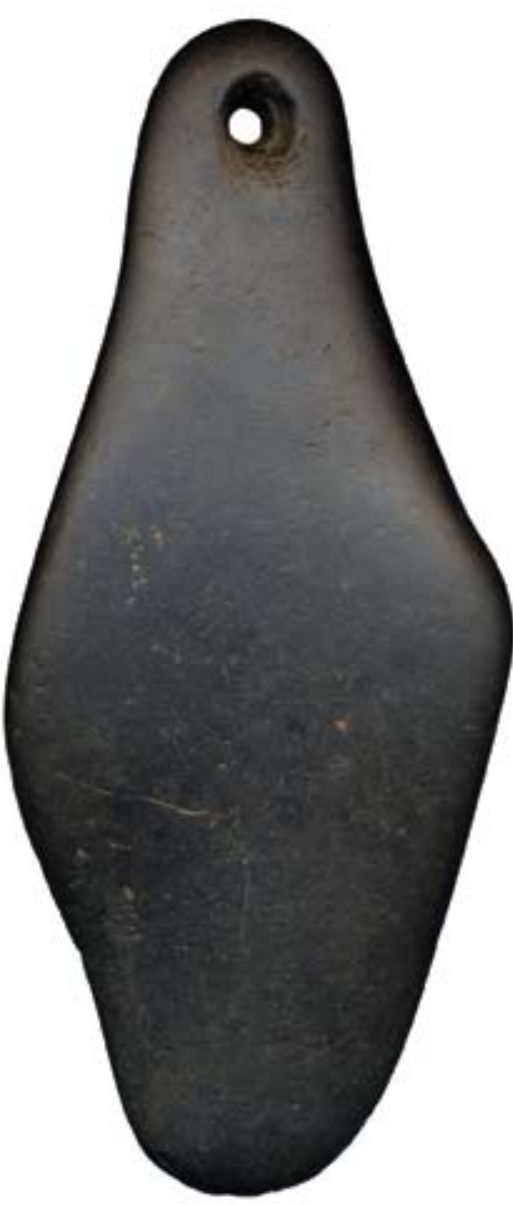

b

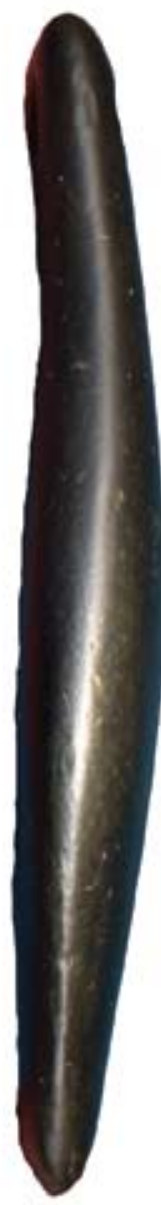

C

1

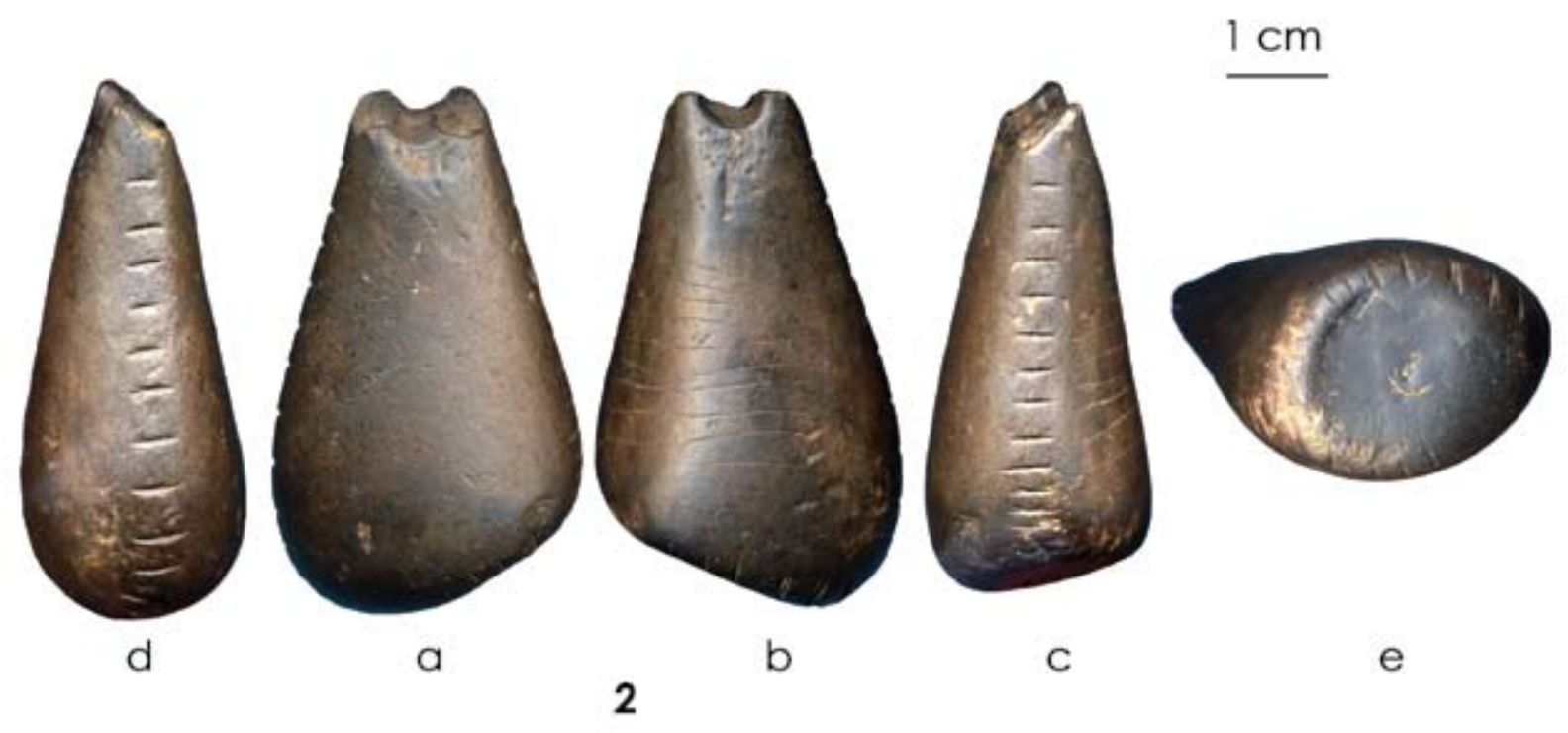

Munibe Monographs. Anthropology and Archaeology Series • 1 (2017) • 453-484 • Donostia-San Sebastián • ISSN 2530-4720 - elSSN $2530-4739$ 

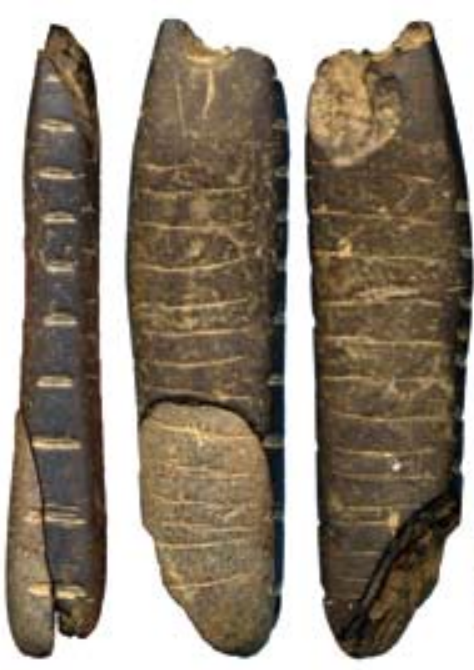

d

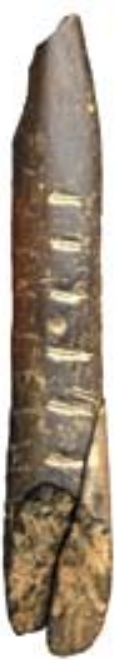

C

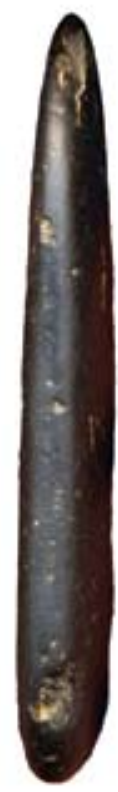

d

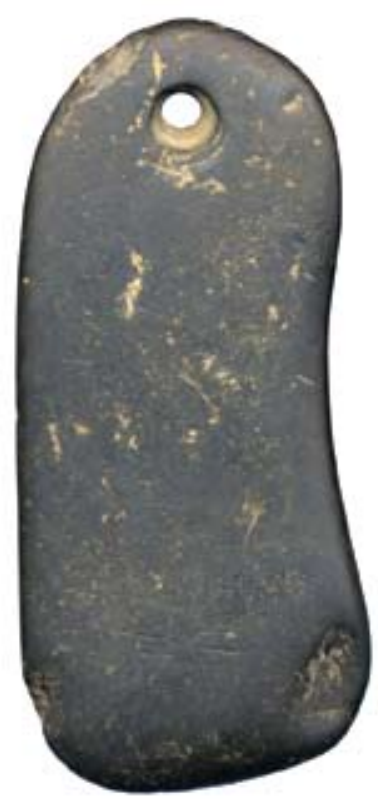

a

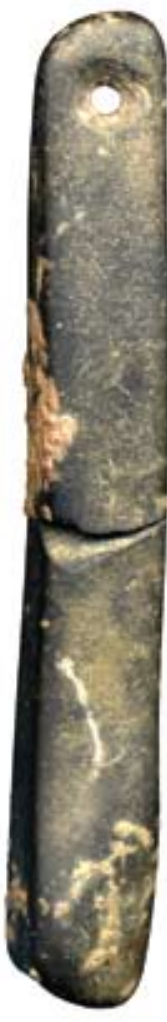

a

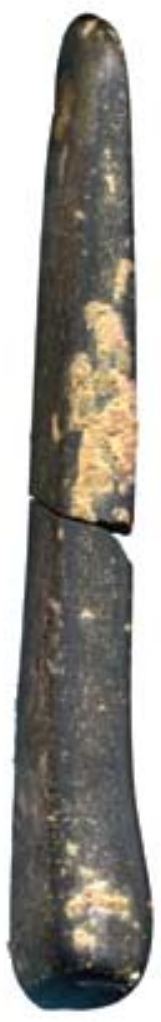

d

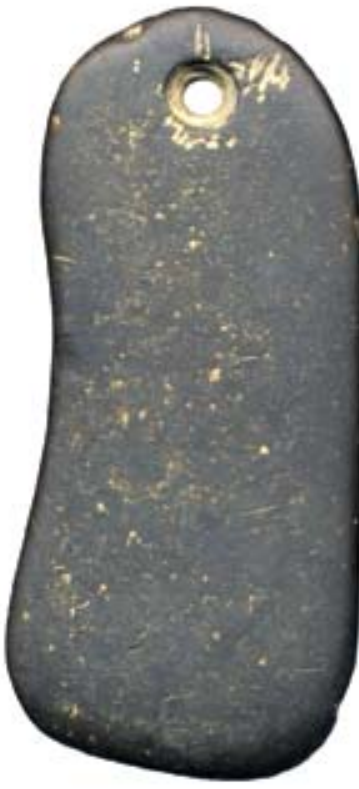

b

4

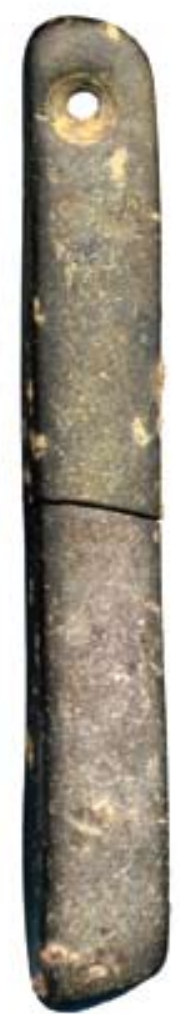

b

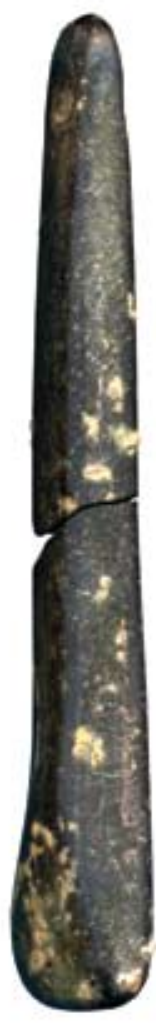

C

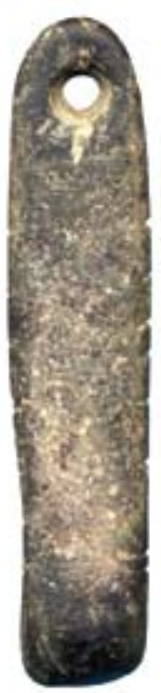

b c

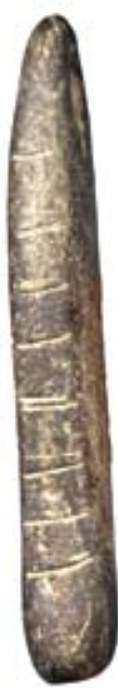

c

8

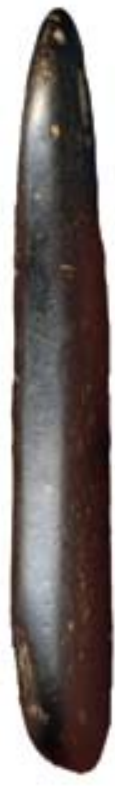

C d

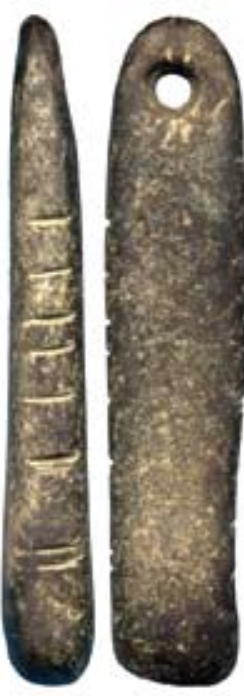

d a

$\mathrm{cm}$ 

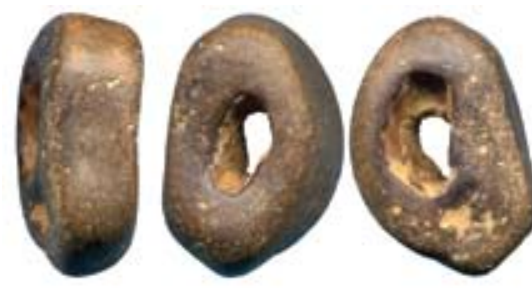

d a

10

$1 \mathrm{~cm}$
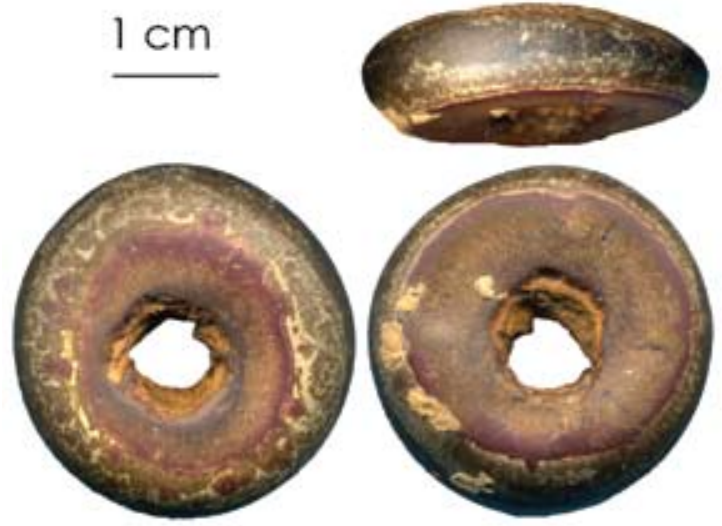

a

12
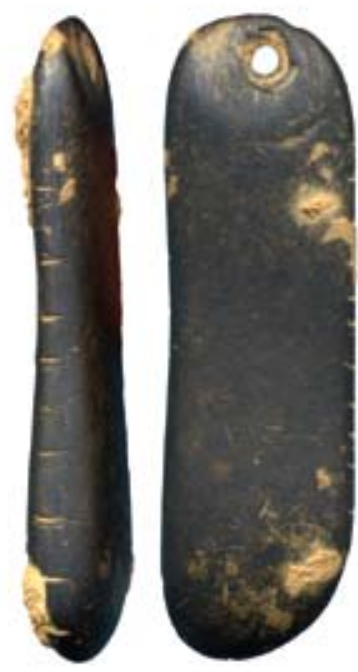

d

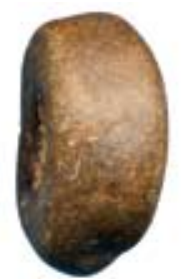

C

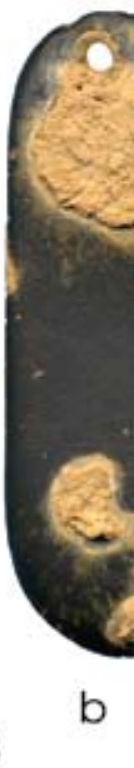

C
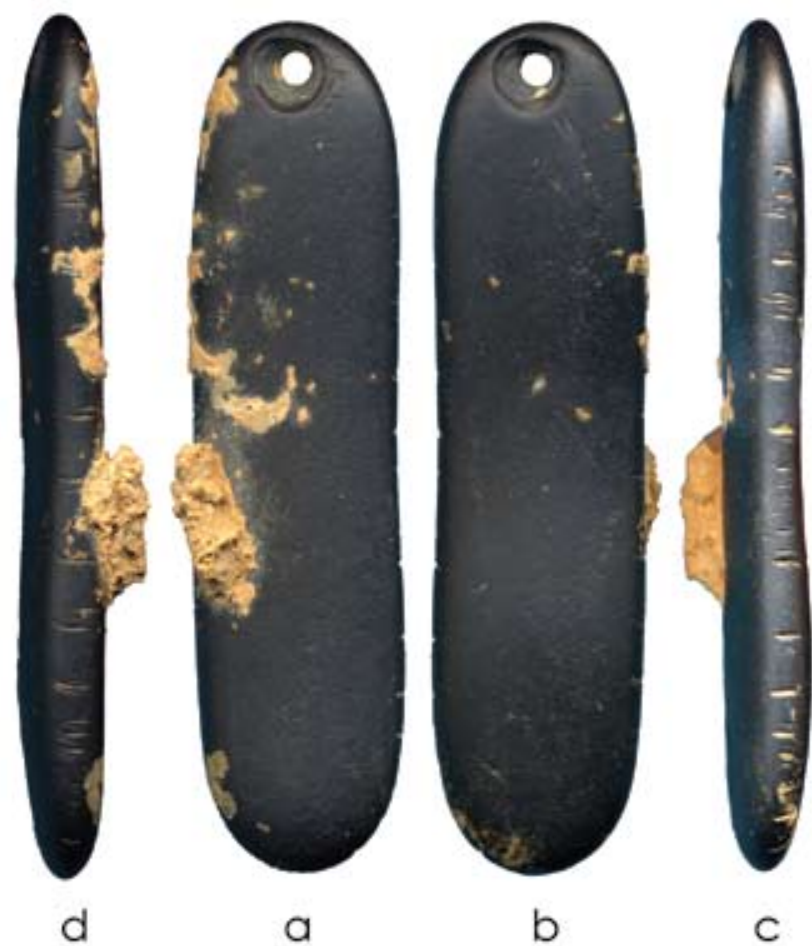

11
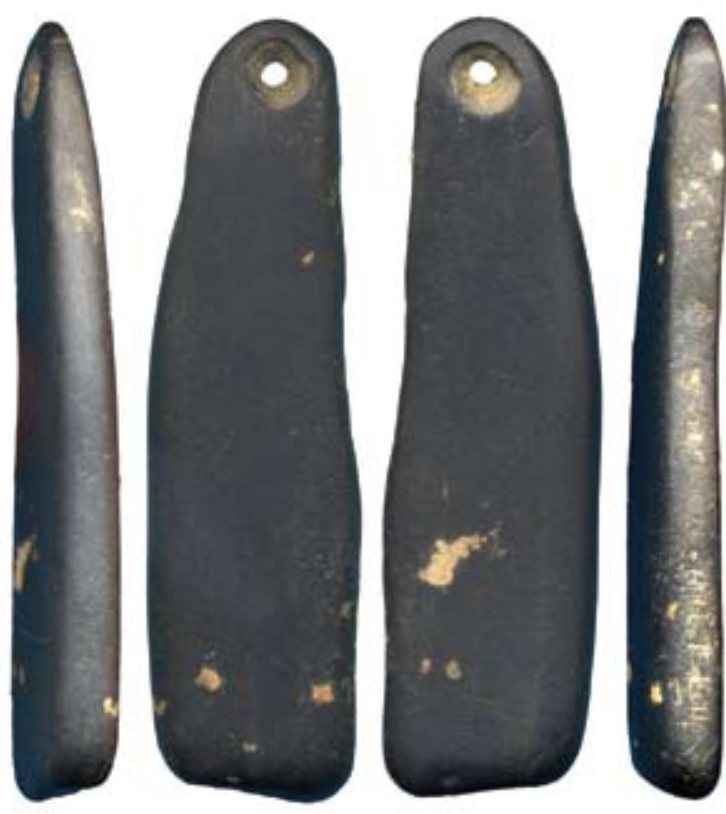

d

a $14 \quad b$

C

13 

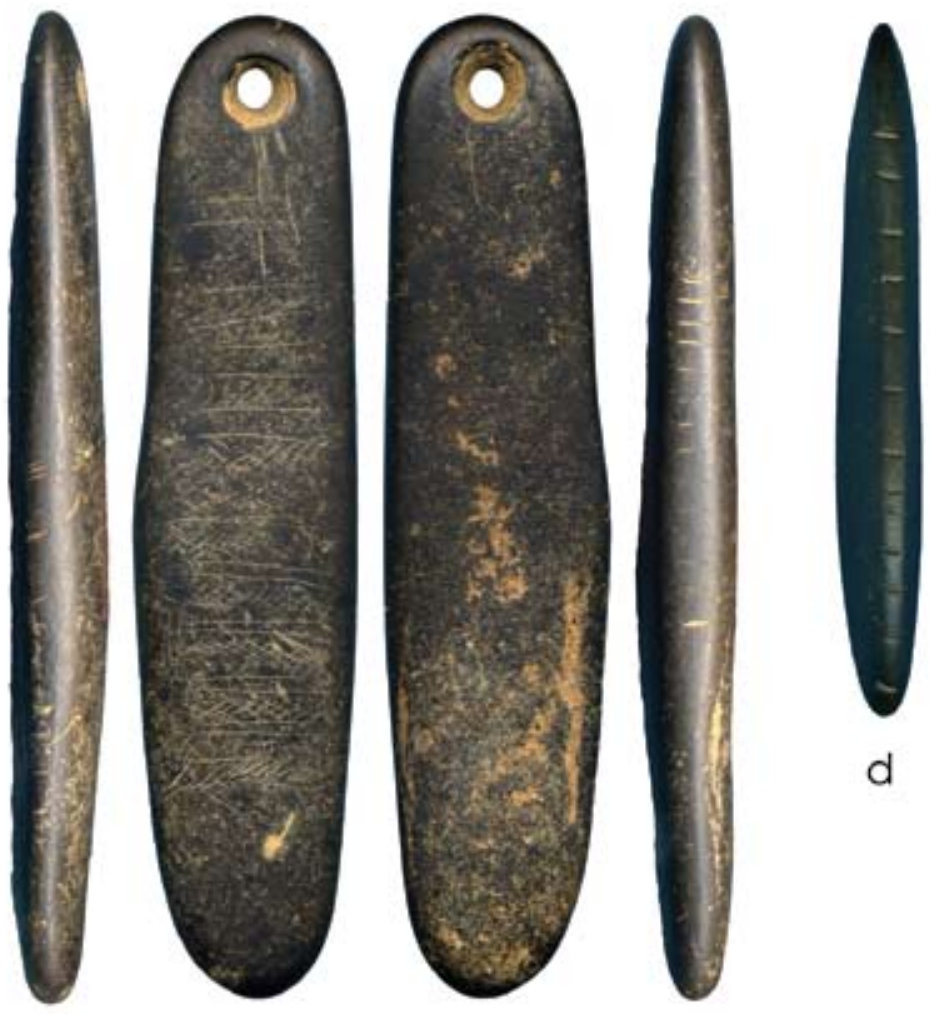

d
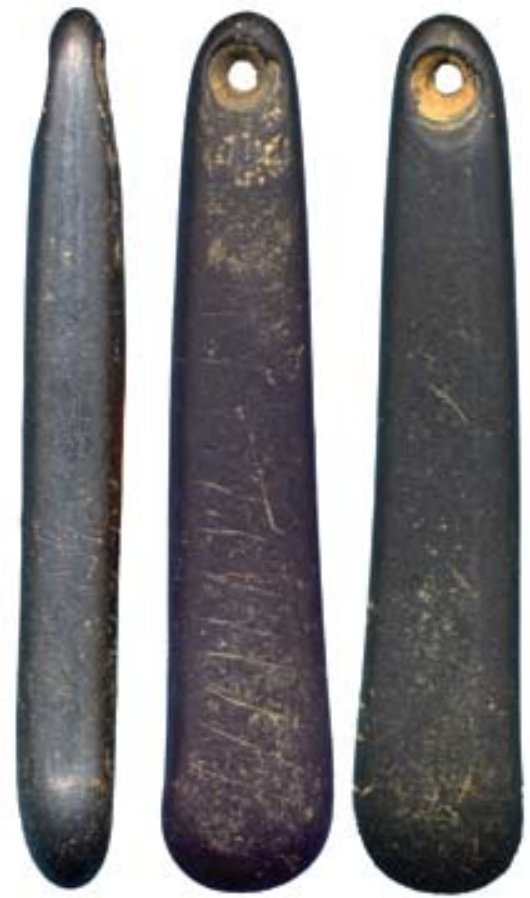

d a

15
C
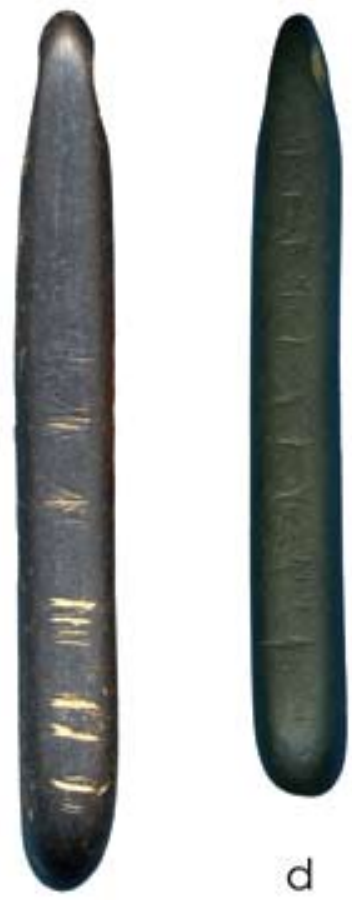

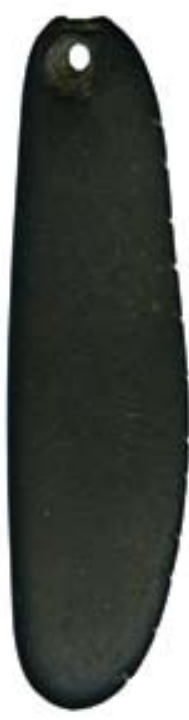

a

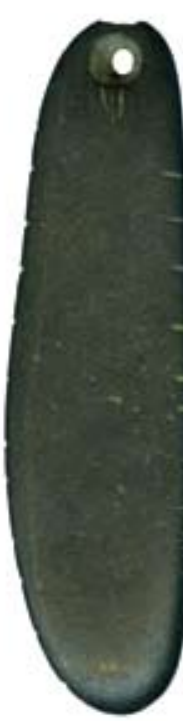

b

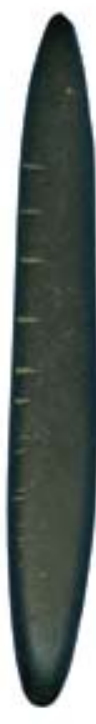

16

$1 \mathrm{~cm}$
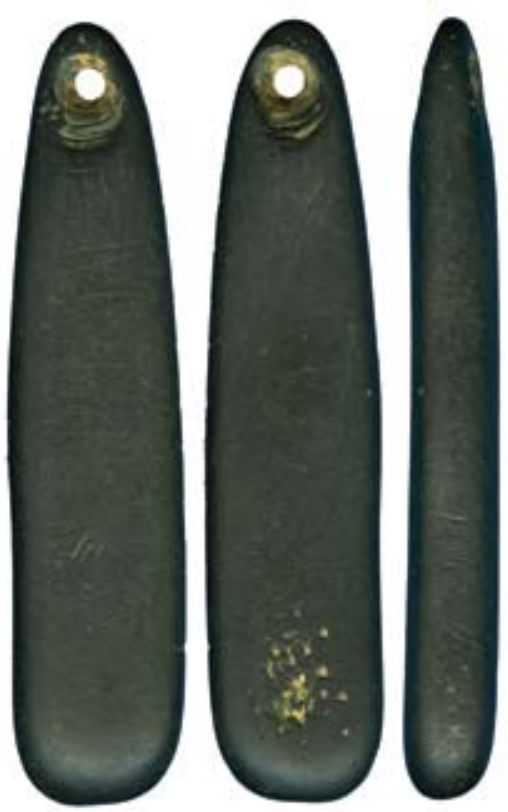

a 18

C 

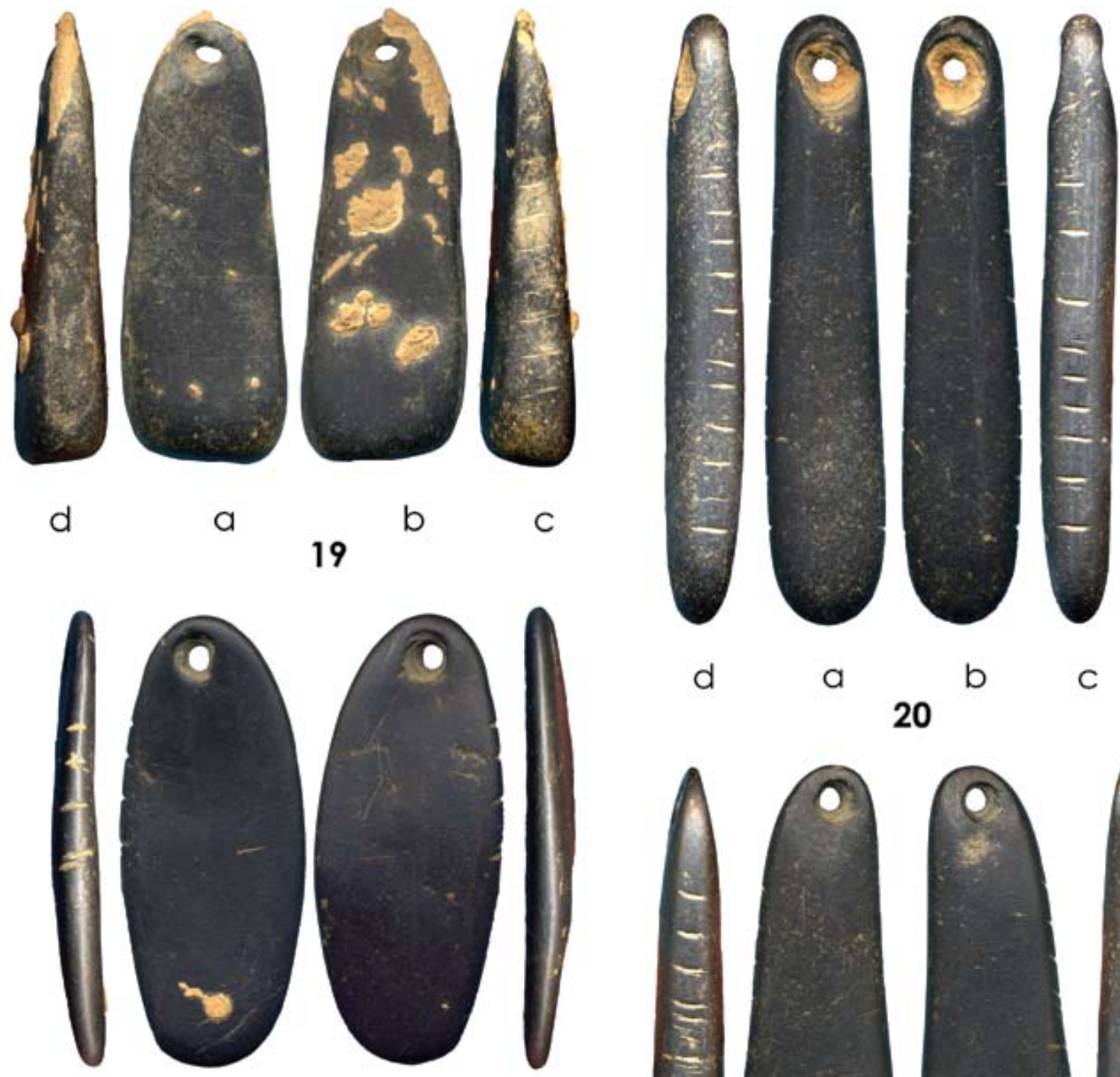

c
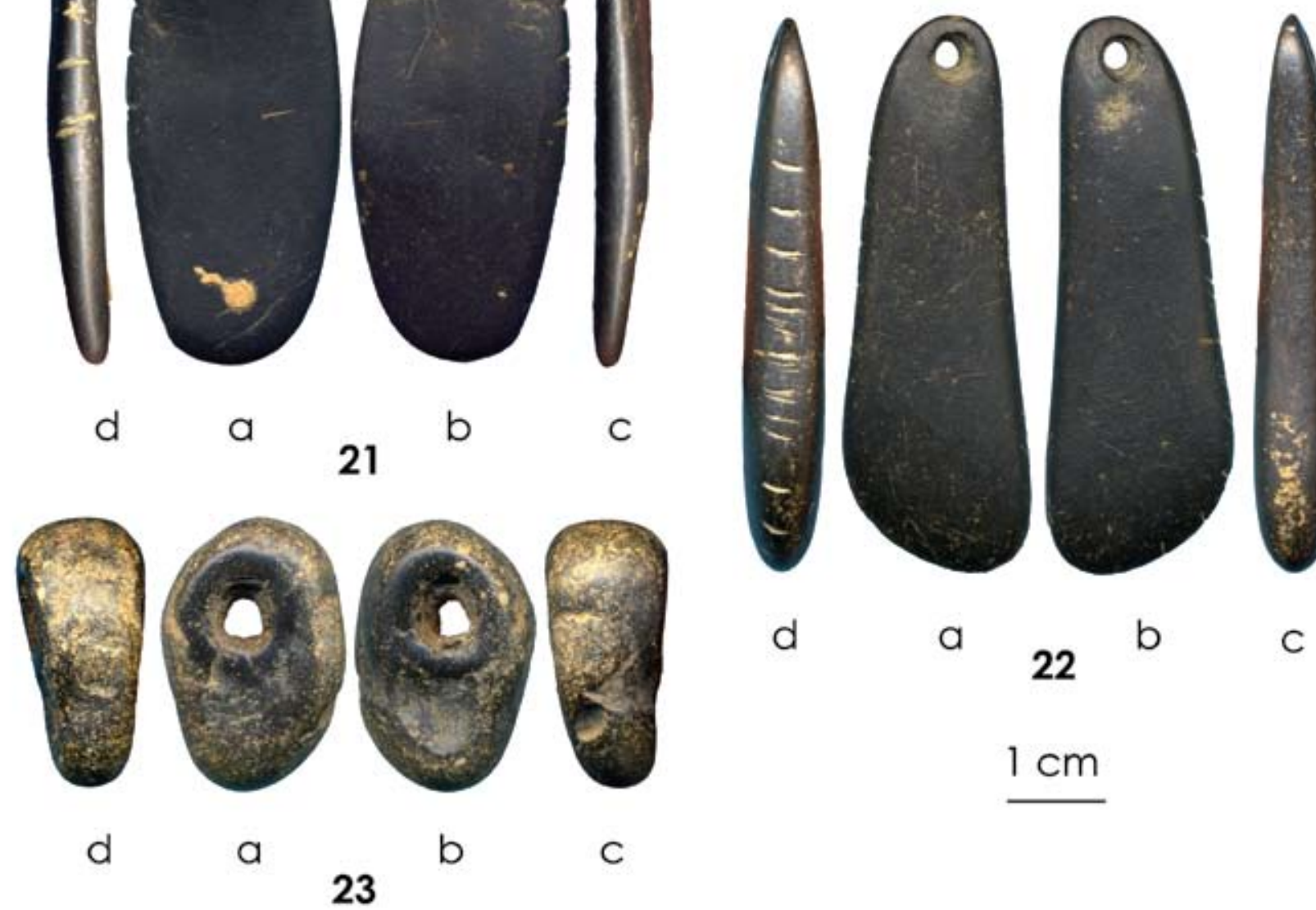

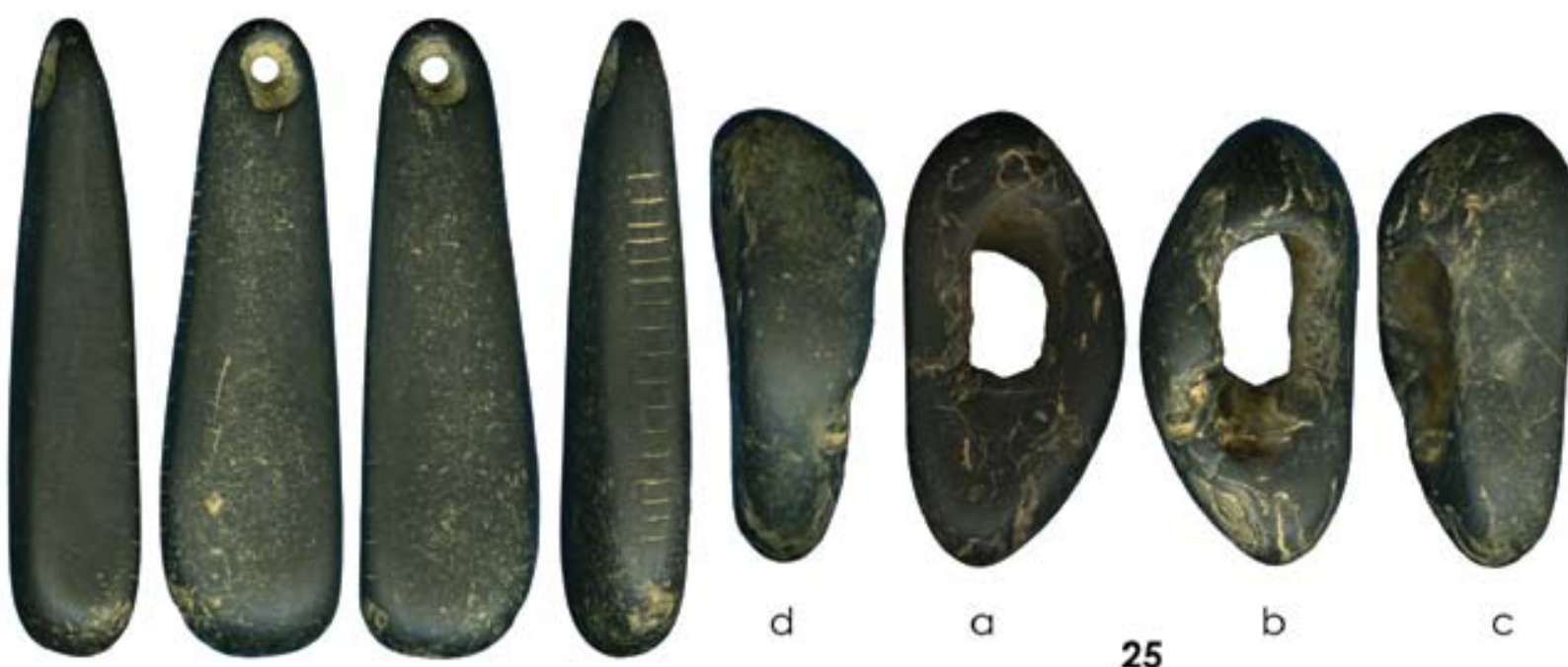

d

a

25

C

d

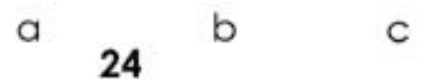

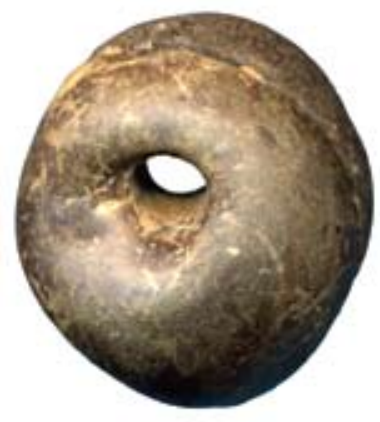

a

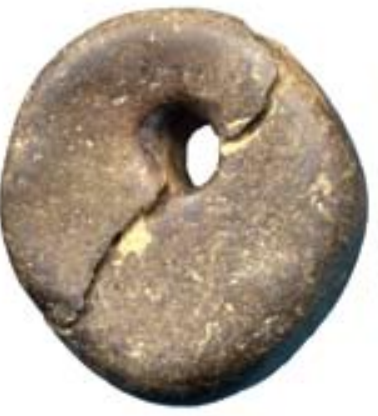

b

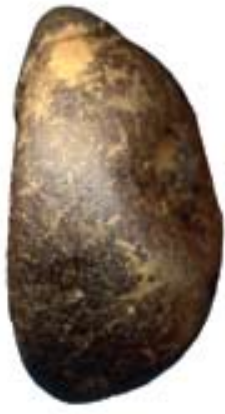

C

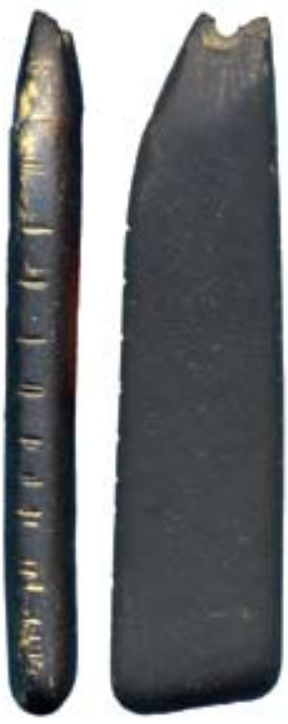

d

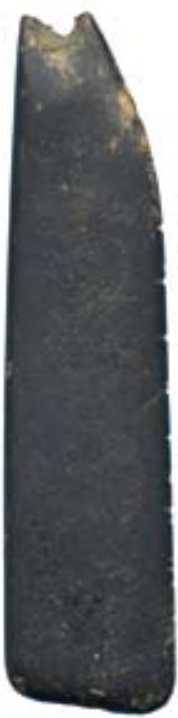

b

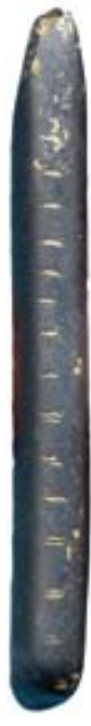

c
$26 a+26 b$

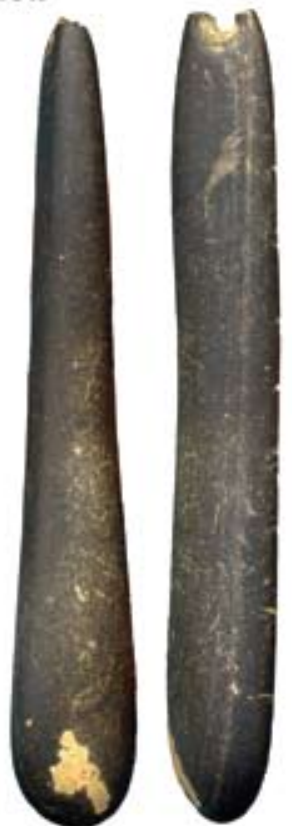

d a

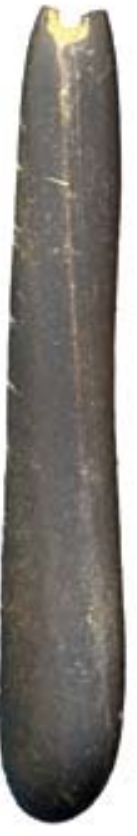

b

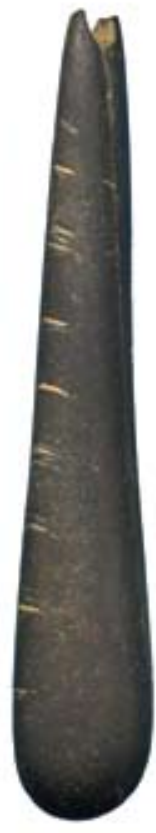

C

28 

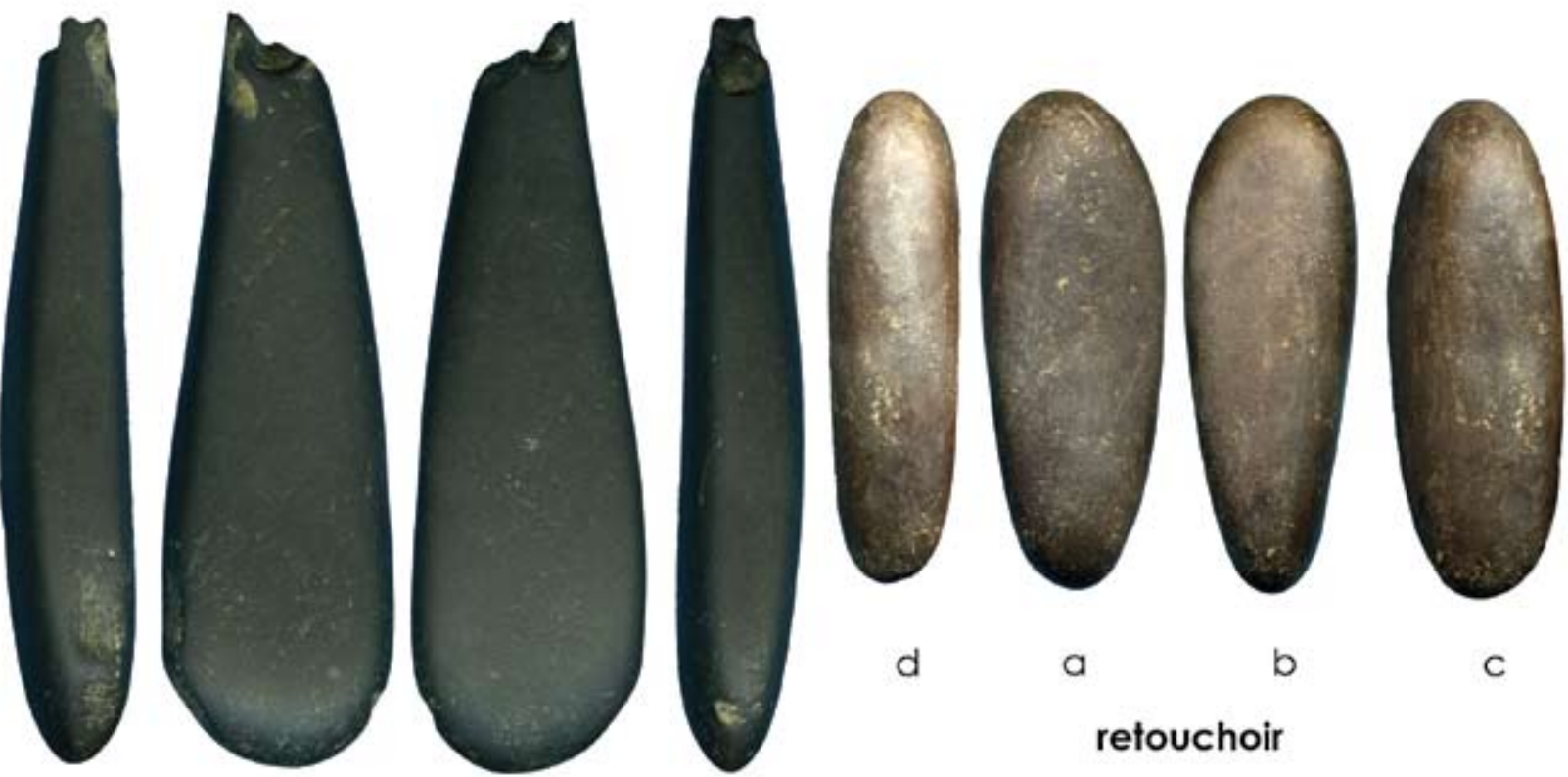

d

a

b

C retouchoir

d

a

b

C

$1 \mathrm{~cm}$ 29

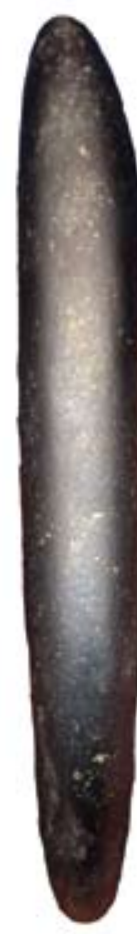

d

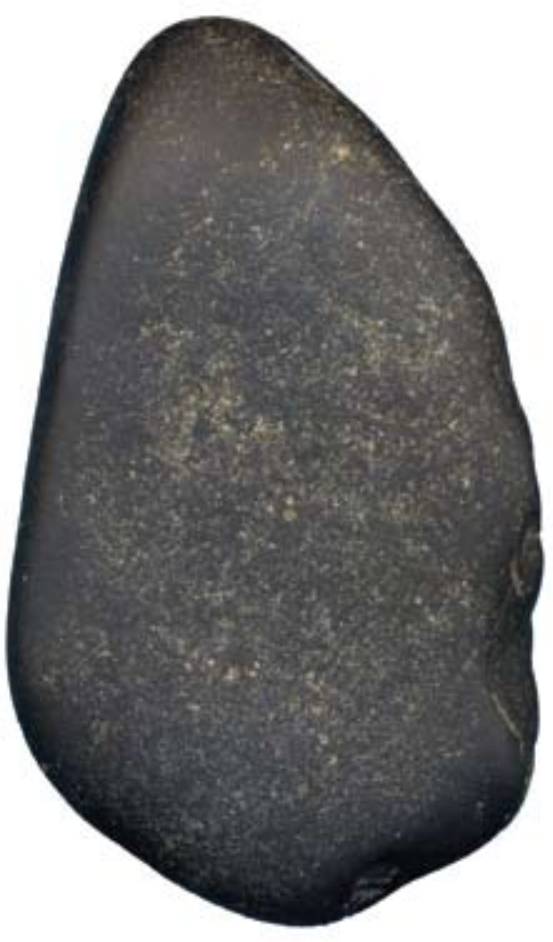

a

non perforé

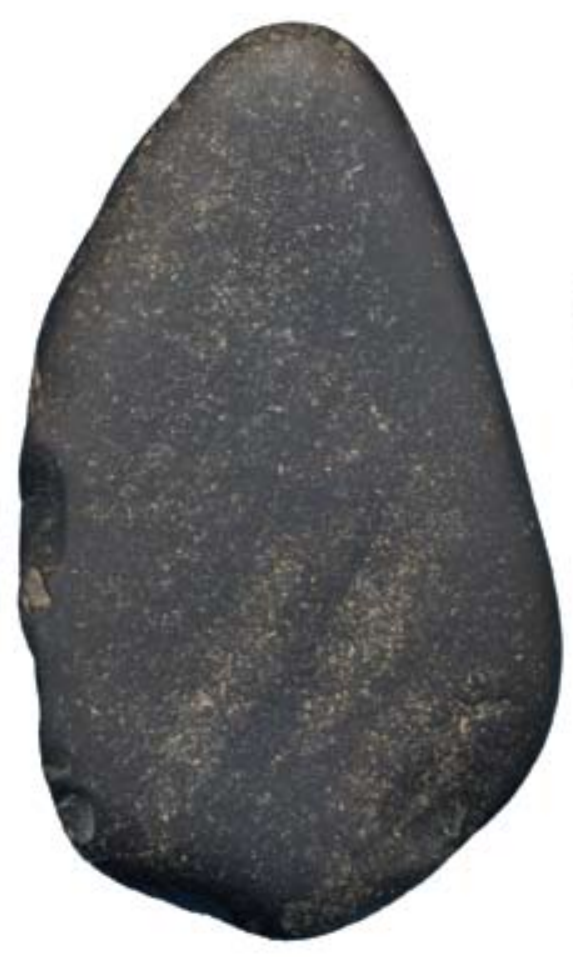

b

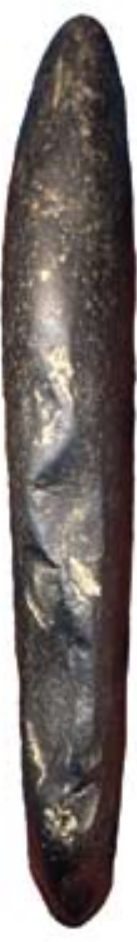

C

Fig. 1-7. Galets exogènes, perforés et non perforés, découverts dans la grotte de Praileaitz I. Les numéros correspondent à ceux du Tabl. 1 , les lettres aux différentes faces des galets. / Pebbles from Praileaitz I. Numbers correspond to those in table 1, letters to the different aspects of the pebbles. 


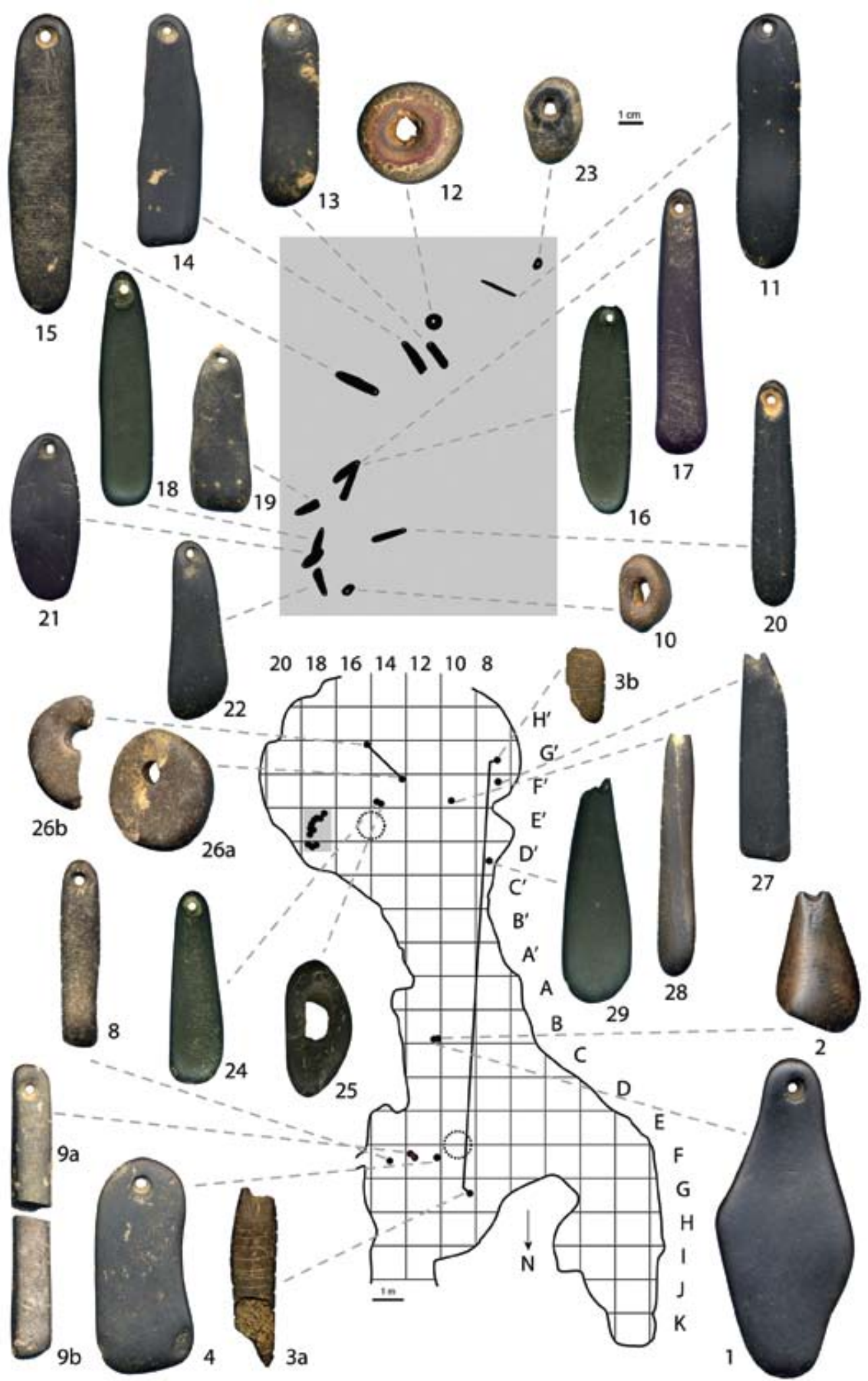

Fig. 8. Localisation des 26 galets perforés (points noirs) découverts dans la grotte de Praileaitz I (les cercles en pointillés représentent les foyers, les deux lignes pleines les remontages, la zone en gris le 'collier'). / Map with the location of the 26 perforated pebbles found at Praileaitz I. Dotted line circles indicate hearths, straight full lines refittings. The area in gray on the plan, enlarged at the top, provides the location of the pebbles belonging to the "necklace". 


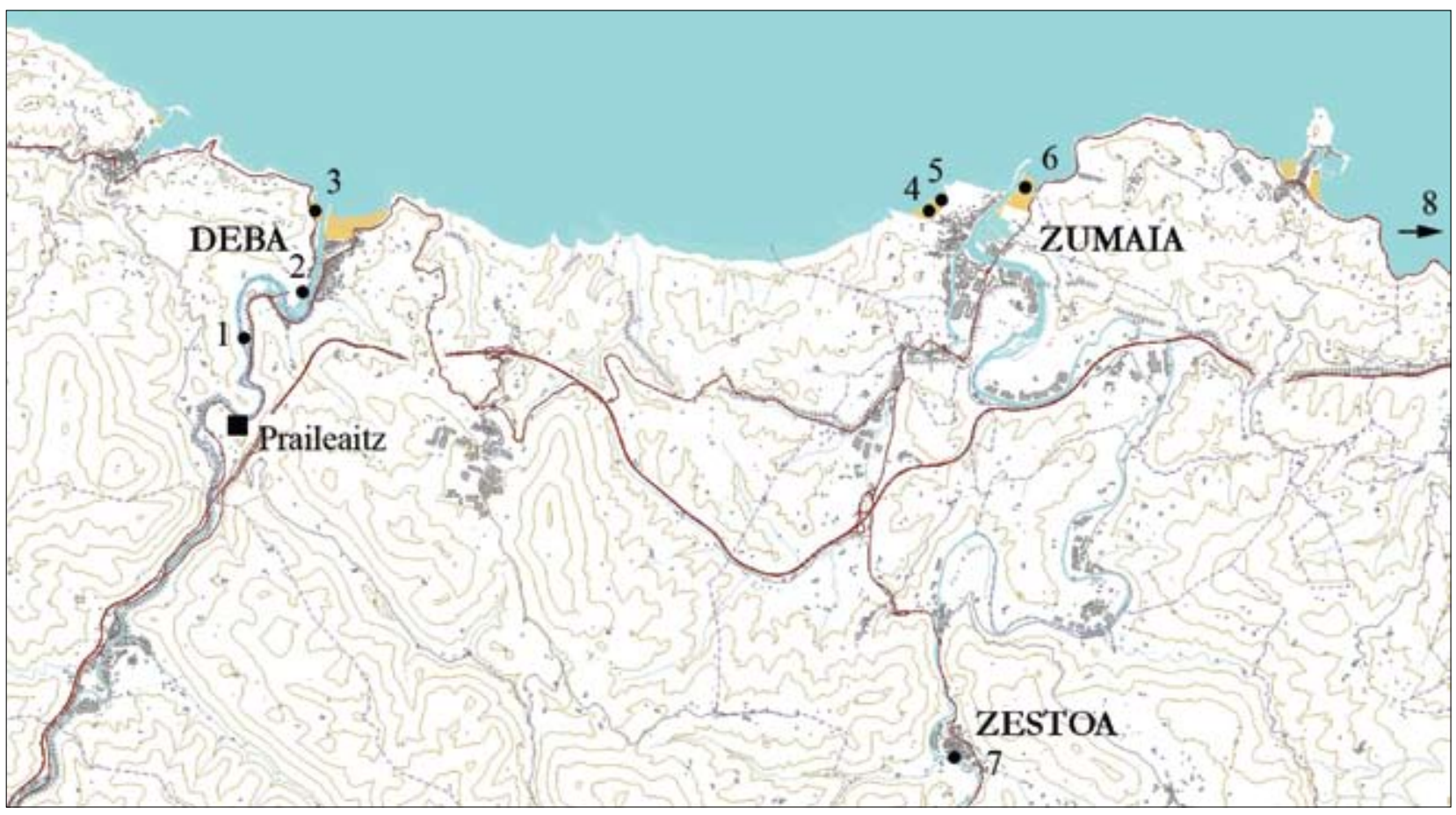

Fig. 9. Localisation de la grotte de Praileaitz I et des huit lieux de collecte des galets constituant la collection de référence. / Map with the location of Praileaitz I and the eight spots where the pebbles of the reference collection were collected.

bablement aussi le cas pour les pièces 1-2 et 24-25. Le remontage entre le vestibule et la première salle de deux fragments de la pièce $n$. 3 et l'utilisation du même type de supports étayent la thèse d'une relative contemporanéité entre les objets découverts dans les deux salles. Les datations ${ }^{14} \mathrm{C}$ AMS (19330 \pm 150 (GrA 24687), $17760 \pm 70 \mathrm{BP}$ (Beta 162879), $15810 \pm 110$ (GrA 24688 B), $15530 \pm 100$ (GrA 24685), $15460 \pm 100$ BP (GrA 20464), $15190 \pm 50$ BP (Beta 162880), $14700 \pm 100$ BP (GrA 20462), PEÑALVER, MUJIKA, 2003, PEÑALVER et al., 2017) obtenues par deux laboratoires sur des esquilles d'os provenant du vestibule et rattachées à la couche ayant livré les galets perforés montre un éparpillement des âges sur presque 5000 ans. Ce dernier est attribué par les fouilleurs au fait que la couche du Magdalénien ancien qui a livré les pendeloques est en contact direct avec la couche solutréenne sous-jacente, ce qui a pu engendrer une attribution stratigraphique erronée de certains restes fauniques.

\section{3.- MATÉRIAUX ET MÉTHODES}

Une prospection a été menée dans trois localités de la vallée du Deba en aval du site de Praileaitz I pour documenter la variabilité minéralogique et morphologique des galets transportés par ce cours d'eau et rechercher des galets semblables à ceux découverts à Praileaitz I (Fig. 9). Le premier lieu de collecte correspond à une petite île

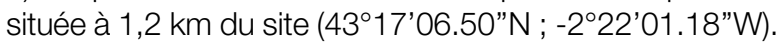
Le deuxième est situé sur une berge localisé à $3,3 \mathrm{~km}$ du site (4317'28.32” N ; -2²1'29.60”W). Le troisième échantillonnage a été effectué sur la plage située à l'ouest de l'estuaire, localisée à 4,3 km du site (4318'01.23" N ; $\left.-2^{\circ} 21^{\prime} 22.11 ” \mathrm{~W}\right)$. Trois localités ont été échantillonnées à proximité de l'estuaire de l'Urola $\left(43^{\circ} 18^{\prime} 3.7^{\prime \prime} \mathrm{N}\right.$; -2¹5'36.7”W; 4318'8.5” N ; -2¹5'28.7"W ; 4318'8.9” N ; $-2^{\circ} 14^{\prime} 46.1^{\prime \prime} \mathrm{W}$ ) et une le long de son cours, près de Zestoa (4314'22" N ; 2 15'26.8”W). À titre comparatif, des galets semblables en couleur à ceux de Praileaitz I ont été également collectés sur la plage de Hendaye $\left(43^{\circ} 21^{\prime} 34.85\right.$ "N ; $1^{\circ} 46^{\prime} 39.70$ 'W). La collectes a été menée par deux personnes pendant environ 20 minutes pour chaque site.

Les quatre faces des galets de Praileaitz I et la plus grande face des galets noirs actuels ont été digitalisées avec un scanner Epson Perfection à une résolution de 1200 dpi. Les galets découverts à Praileaitz I ont été aussi photographiés. L'image digitalisée de la plus grande face de chaque galet a été analysée avec le logiciel d'analyse d'image ImageTool 3.00 (The University of Texas Health Science Center, San Antonio), ce qui a permis d'obtenir la mesure de la surface, du périmètre, de l'axe majeur, mineur, des indices d'allongement et de circularité de chaque pièce. L'épaisseur a été mesurée avec un pied à coulisse digital. Dans le cas des galets archéologiques fracturés, le profil original a été reconstitué en tenant compte de la morphologie naturelle des galets et, pour les pièces avec perforation fracturée, en s'appuyant également sur la morphologie de la perforation. Le diamètre maximal et minimal des perforations et la distance entre le bord interne de la perforation et l'extrémité du galet la plus proche dans l'axe d'allongement de celui-ci ont été mesurés avec un pied à coulisse digital. La composition 
élémentaire des galets archéologiques et des galets noirs actuels a été établie de manière non destructive en utilisant un spectromètre à fluorescence $X(E D-X R F)$ portatif SPECTRO xSORT. Cet appareil utilise comme source d'excitation un tube à rayons $X$ de $40 \mathrm{kV}$ et $50 \mu \mathrm{A}$ à anticathode de tungstène et un détecteur de type SDD. Les mesures sont acquises dans une station d'accueil métallique permettant une mesure en toute sécurité. Le xSORT est fixé à cette station et analyse l'objet par en dessous, ce qui évite de charger l'objet avec le poids de l'appareil et élimine les risques de frottement. Deux ou trois mesures de 60 secondes ont été acquises pour chaque galet. Les spectres ainsi obtenus ont été étudiés dans le logiciel XlabPRO. L'appareil n'étant pas calibré pour ce type de matériau, les teneurs des différents éléments visibles sur le spectre ont été calculées selon leur nombre de coups (surface du pic). Une comparaison de la composition chimique des galets est également possible par la présence ou l'absence des pics caractéristiques des éléments directement sur les spectres mesurés par l'appareil. Le pic de tungstène $(W$ ) est lié à l'anticathode du dispositif de mesure tandis que le pic d'argon (Ar) est lié à la présence de cet élément dans l'air.

Les galets perforés archéologiques ont été observés à la loupe binoculaire pour documenter toute trace d'intervention anthropique. Ces traces ont été photographiées à l'aide d'une loupe binoculaire Wild M3C équipée d'une caméra digitale Nikon Coolpix 995. Les images digitales des objets de parure ont servi comme support pour enregistrer la localisation et la nature des traces microscopiques identifiées. Un échantillon de galets naturels les plus semblables en couleur et composition aux pendeloques en pierre de Praileaitz I a été également observé à la loupe binoculaire pour documenter la variabilité des états de surface. Les caractères microscopiques permettant de reconstituer la démarche de l'artisan (technique de perforation et gravure, direction du mouvement de l'outil, ordre des gravures, utilisation du même outil et points correspondant aux changements d'outil) et l'utilisation des objets (usures liées au port des objets, impacts d'utilisation, fractures, traces de pigment, etc.) ont été également repérés et documentés photographiquement.

\section{4.- RÉSULTATS}

\subsection{Morphologie}

D'un point de vue morphologique, la collection de Praileaitz I est majoritairement composée de deux types de galets : des galets allongés ou très allongés et des galets de forme circulaire ou sub-circulaire avec une perforation naturelle (Figs. 1-7, Tabl. 1). La grande majorité des pièces appartenant à la première catégorie rentre dans la variabilité morphologique de galets noirs de notre collection de référence (Fig. 10 et 11). Une différence s'observe par contre entre les galets à perforation anthropique du «collier » et ceux découverts ailleurs dans le site, les premiers ayant une plus forte homogénéité quant à leur largeur et épaisseur.

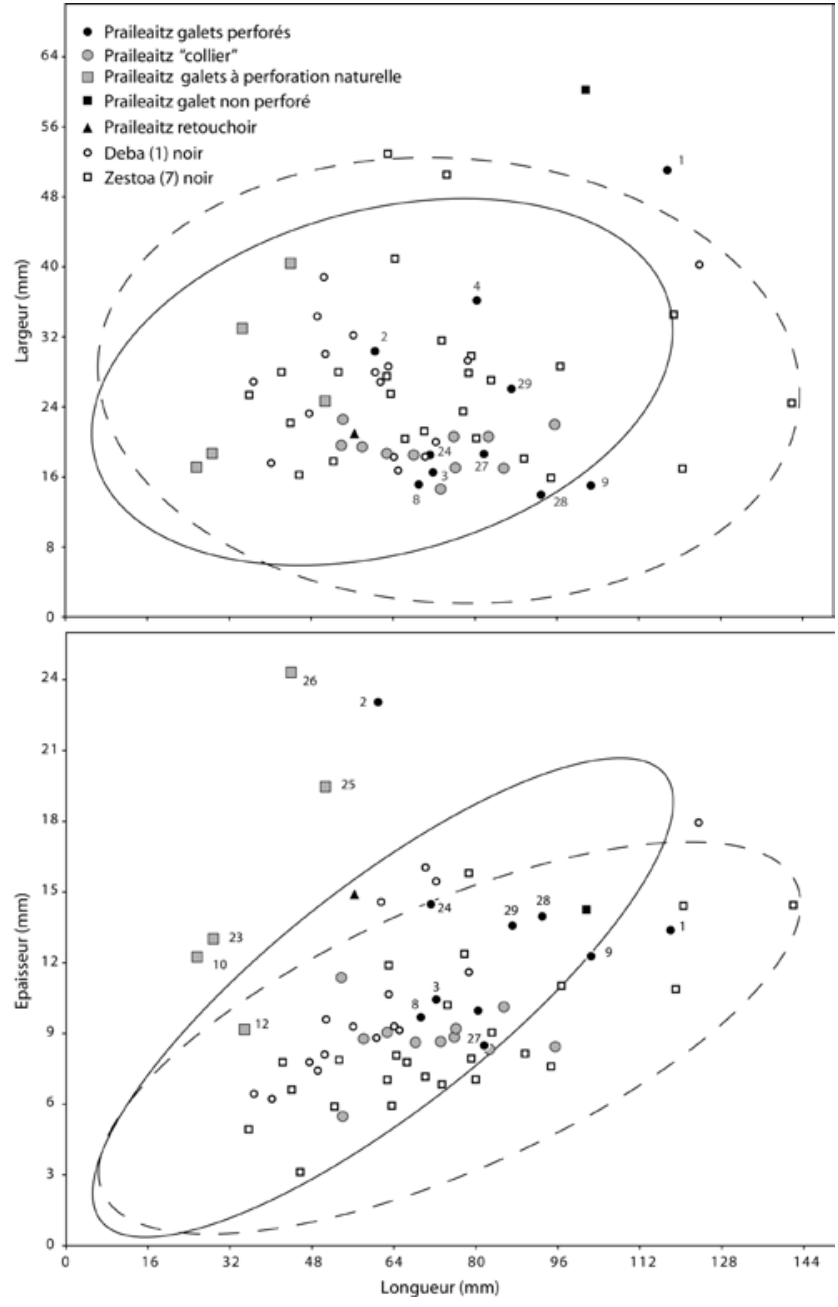

Fig. 10. Dimension des galets perforés de Praileaitz I et des galets noirs collectés sur les plages à galets du Deba (lieu de collecte $n$. 1) et de l'Urola (lieu de collecte n. 7, près de la ville de Zestoa). Ellipses de confiance $=95 \%$ (ligne pleine : Deba; ligne pointillée: Zestoa). / Dimensions of the Praileaitz I perforated pebbles and the black pebbles collected on the Deba (spot n. 1) and the Urola (spot n. 7, close to the town of Zestoa) riverbanks. Confidence ellipse $=95 \%$ (full line: Deba; dotted line: Zestoa).

Aucun galet appartenant à la deuxième catégorie, celle des galets à perforation naturelle, n'a été rencontré dans nos prospections. Une seule pièce (Fig. 12) présentant un début de perforation naturelle et un état de surface semblable à celui du galet $n^{\circ} 12$ a été trouvée dans plage à galet du Deba (lieu de collecte n. 1).

\subsection{Composition élémentaire}

Les galets noirs de Praileaitz I sont constitués d'une roche cryptocristalline mélanocrate qui ne présente pas de schistosité apparente. II s'agit vraisemblablement d'une lutite, peut-être issue du démantèlement de certains bancs compacts et fins de flysch, ultérieurement transformés en galets par la mer. 
- Praileaitz galets perforés

- Praileaitz "collier"

$\square$ Praileaitz galets à perforation naturelle

- Praileaitz galet non perforé

A Praileaitz retouchoir

- Deba (1) noir

- Zestoa (7) noir
Fig. 11. Analyse en composante principale sur la largeur, la surface, le périmètre et les indices d'allongement et de circularité des galets perforés de Praileaitz I et des galets noirs collectés le long des rivières du Deba (lieu de collecte n. 1) et de l'Urola (lieu de collecte n. 7 près de la ville de Zestoa). Ellipses de confiance $=95 \%$ (ligne pleine : Deba; ligne pointillée : Zestoa). / PCA scatterplot of unperforated, and deliberately and naturally perforated pebbles from Praileaitz as well as black pebbles collected along the Deba (spot n. 1) and the Urola (spot n. 7, close to the town of Zestoa) riverbanks. Considered variables are width, surface, perimeter, index of elongation and circularity. Confidence ellipse = 95\% (full line: Deba; dotted line: Zestoa).

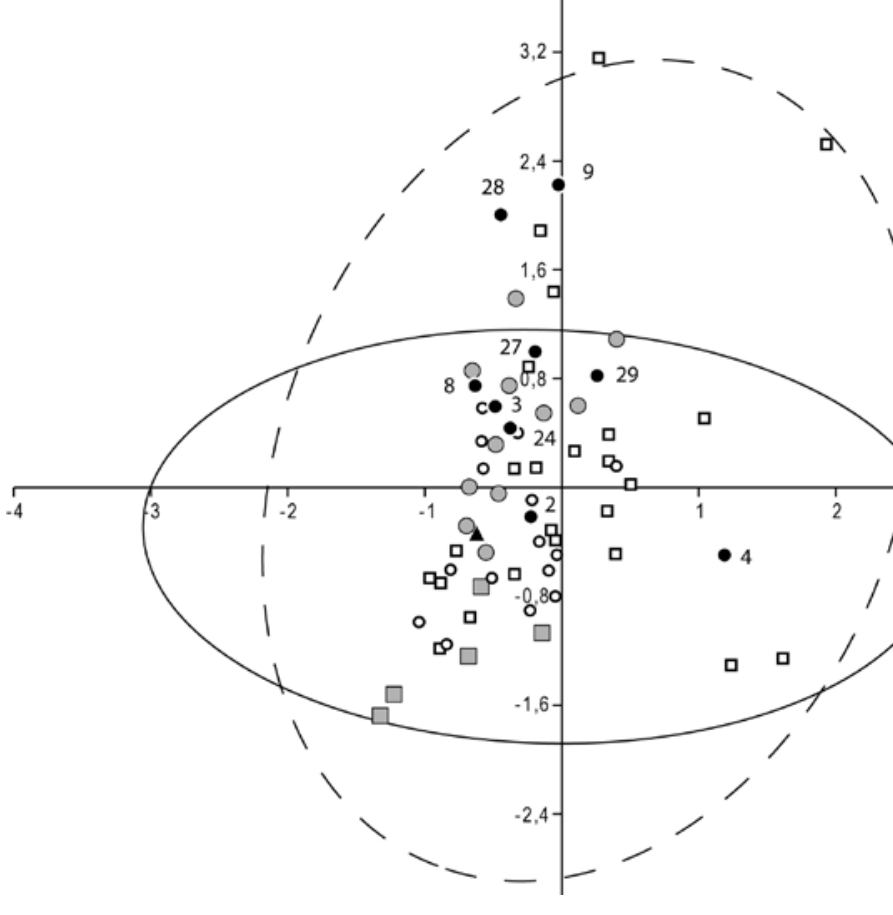

Les analyses XRF montrent que ces galets sont majoritairement composés de $\mathrm{SiO}_{2}$ et $\mathrm{Fe}_{2} \mathrm{O}_{3}$. D'autres éléments (dans l'ordre croissant de masse atomique : $\mathrm{Al}, \mathrm{K}$, $\mathrm{Ca}, \mathrm{Ti}, \mathrm{V}, \mathrm{Cr}, \mathrm{Mn}, \mathrm{Ni}, \mathrm{Zn}, \mathrm{V}, \mathrm{Rb}, \mathrm{Sr}, \mathrm{Y}, \mathrm{Zr}$ ) sont présents en faible proportion (Fig. 13). La variabilité des compositions en fer et calcium impactant la variabilité globale des compositions, nous avons extrait ces deux éléments de l'analyse multivariée et les avons utilisés dans une analyse spécifique.

Les galets archéologiques avec des perforations anthropiques ont un rapport fer/calcium comparable et clairement différent de celui de nos référentiels (Fig. 14). Ces derniers se caractérisent aussi par une plus forte variabilité dans la proportion de ces deux éléments. Les deux galets non perforés ont une composition semblable à ceux qui portent des perforations anthropiques. Parmi les galets à perforation naturelle, le n. 25 et en moindre mesure le $\mathrm{n}$. 10, présentent un ratio fer/calcium semblable à celui des galets à perforation anthropique. Les galets $n$. 26 et n. 23, par contre, ont un ratio fer/calcium proche de certains galets noirs provenant de la vallée du Deba, de I'Urola et de la Bidasoa. Le galet n. 12, composé de trois couches, constitue un cas particulier. Les deux couches

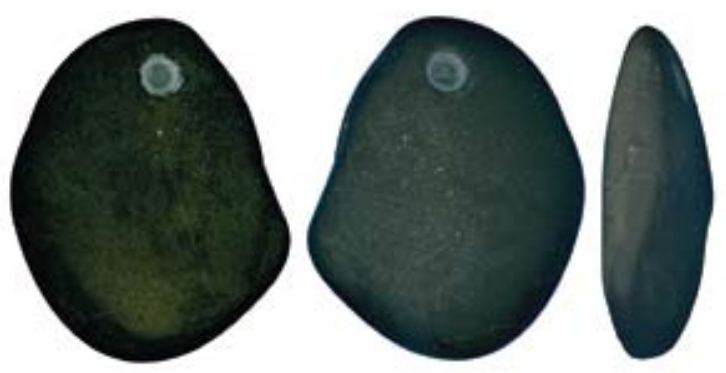

Fig. 12. Haut : Galet avec début de perforations naturelles collecté sur une plage à galet du Deba (lieu de collecte n. 1). Bas : Galet collecté sur la plage de Hendaye (lieu de collecte n. 8) et perforé expérimentalement. / Top: pebble with incipient natural perforation collected on the Deba riverbank (spot n. 1). Bottom: pebble collected on the beach at Hendaye (spot n. 8) and perforated experimentally. 

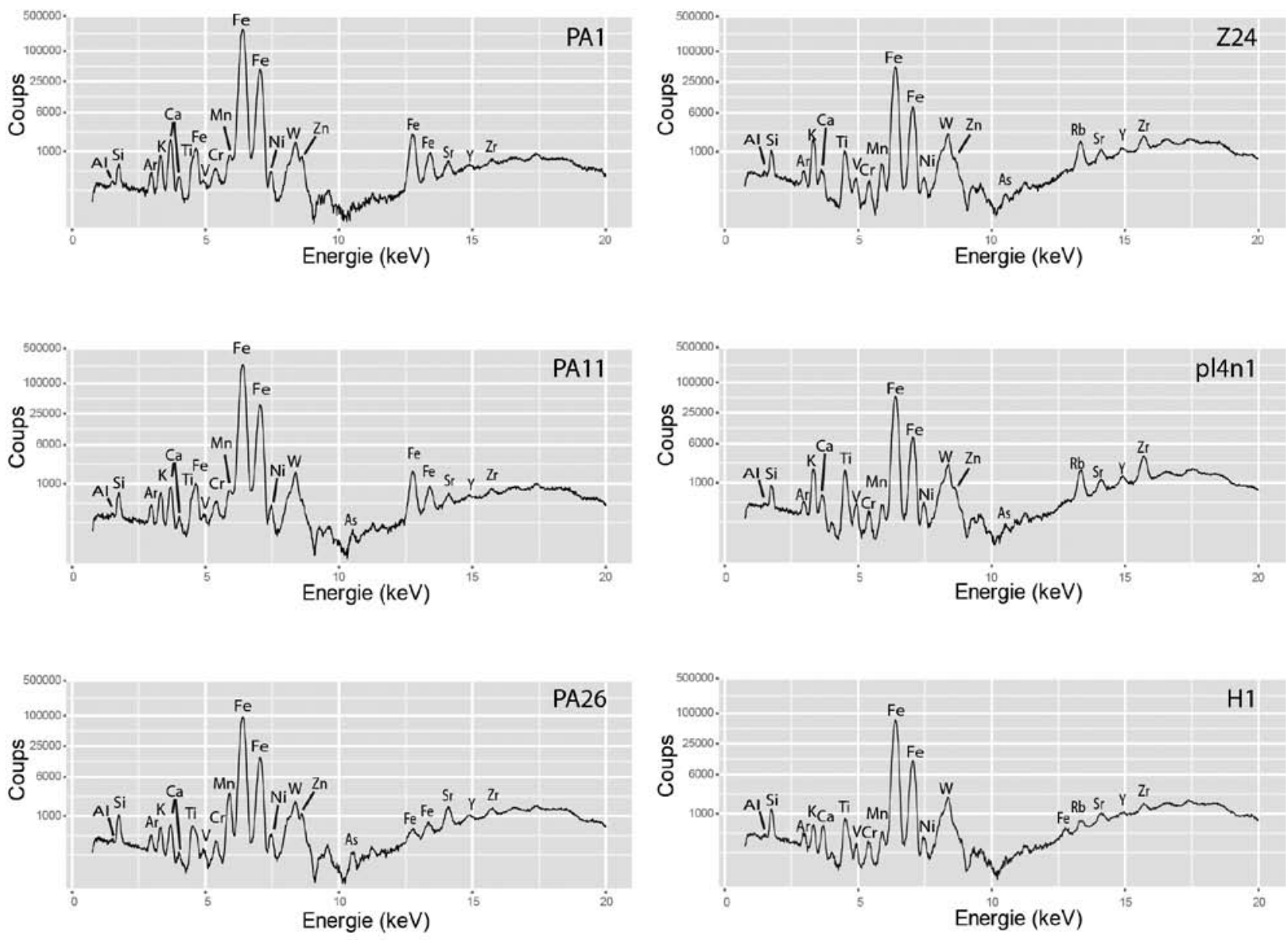

Fig. 13. Spectres XRF de trois galets perforés de Praileaitz I (n. 1, 11 et 26), d'un galet noir collecté sur une plage à galets du Deba (lieu de collecte n. 1, pl4n1), d'un galet noir collecté sur une plage de l'Urola (lieu de collecte n. 7, près de la ville de Zestoa, Z24) et d'un galet noir collecté sur une plage de l'estuaire du Bidasoa (lieu de collecte n. 8, près de la ville de Hendaye, H1). / XRF spectra of three perforated pebbles from Praileaitz I (n. 1, 11 et 26), three black pebbles collected on the Deba riverbank (spot n. 1, pl4n1), the Urola riverbank (spot $n$. 7, close to the town of Zestoa, Z24), and along the Bidasoa estuary (spot n. 1, close to the town of Hendaye, H1).

externes, riches en fer, le différencient des galets perforés, la couche centrale a la même composition que ces derniers. Cette structure en couches, non retrouvée par ailleurs, appuie l'hypothèse d'une origine des galets dans un système sédimentaire comme le flysch.

L'analyse en composantes principales des autres éléments confirme l'homogénéité de l'échantillon archéologique (Fig. 15). Les seuls galets qui s'éloignent quelque peu de la tendance dominante $(n .8,9)$ ont à leur surface des micro-dépôts qui ont pu légèrement biaiser les analyses XRF ou appartiennent à la catégorie des galets à perforation naturelle (n. 26). Les galets de la vallée du Deba et de l'Urola ont une composition différente de celle des galets archéologiques et montrent comparativement une plus forte variabilité dans la teneur des éléments minoritaires. Lorsque l'on combine l'analyse des deux éléments majeurs et celle des éléments mineurs ou en trace, aucun galet naturel n'entre véritablement dans la variabilité des galets archéologiques.

\subsection{Perforation}

Vingt-et-un galets de Praileaitz I ont été perforés par les préhistoriques (Tabl. 2). Cinq autres (n. 10, 12, $23,25,26)$ portent des perforations naturelles qui ont pu être partiellement vidées de leur contenu (sédiment plus au moins concrétionné) pour rendre la perforation utilisable. L'étude microscopique des perforations anthropiques a mis en évidence l'emploi de quatre techniques destinées à préparer la zone à perforer et une seule, la rotation bipolaire à la main, utilisée ensuite pour la perforation proprement dite. Les techniques de préparation comprennent l'incision d'un ou plusieurs sillons (Figs. 16a,e,f, 17a,b,e,f, 18b,c,f, 20c, 21a,b), le piquetage (Fig. 16), le râclage (Figs. 19c,d) et l'abrasion (Figs. $17 \mathrm{~b}, 18 \mathrm{a}, \mathrm{b}, 21 \mathrm{a}, \mathrm{b})$. Les trois premières techniques ont été appliquées avec un outil lithique pointu, la dernière en frottant l'extrémité du galet contre un support abrasif tel qu'une plaquette de grès ou de quartzite. La première de ces techniques est destinée à caler la pointe 


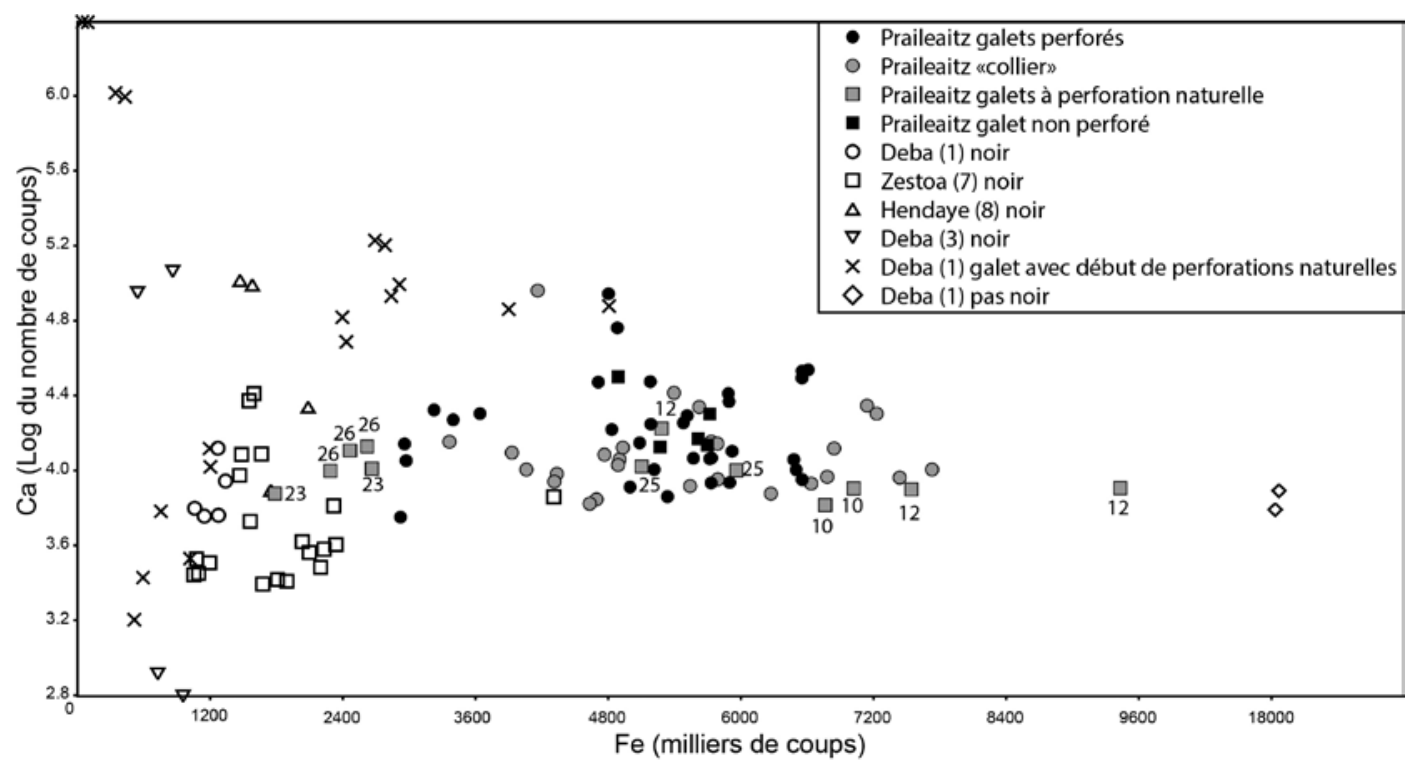

Fig. 14. Résultats de l'analyse XRF pour le fer ( $\mathrm{Fe}$ ) et le calcium (Ca) pour les galets de Praileaitz I, pour les galets noirs, pas noirs et un galet à perforation naturelle collecté le long du Deba (lieux de collecte n. 1 et 3), pour les galets noirs collectés sur la terrasse fluviale de l'Urola (lieu de collecte n. 7, près de Zestoa) et pour les galets noirs de la plage de Hendaye (lieu de collecte n. 8). / Iron (Fe) and Calcium (Ca) concentrations in pebbles from Praileaitz I, black, non-black and naturally perforated pebbles collected on the Deba riverbank (spot $\mathrm{n}$. 1 and 3), black pebbles from the Urola (spot n. 7, close to the town of Zestoa) riverbank, and black pebbles from the Hendaye beach (spot n. 8).

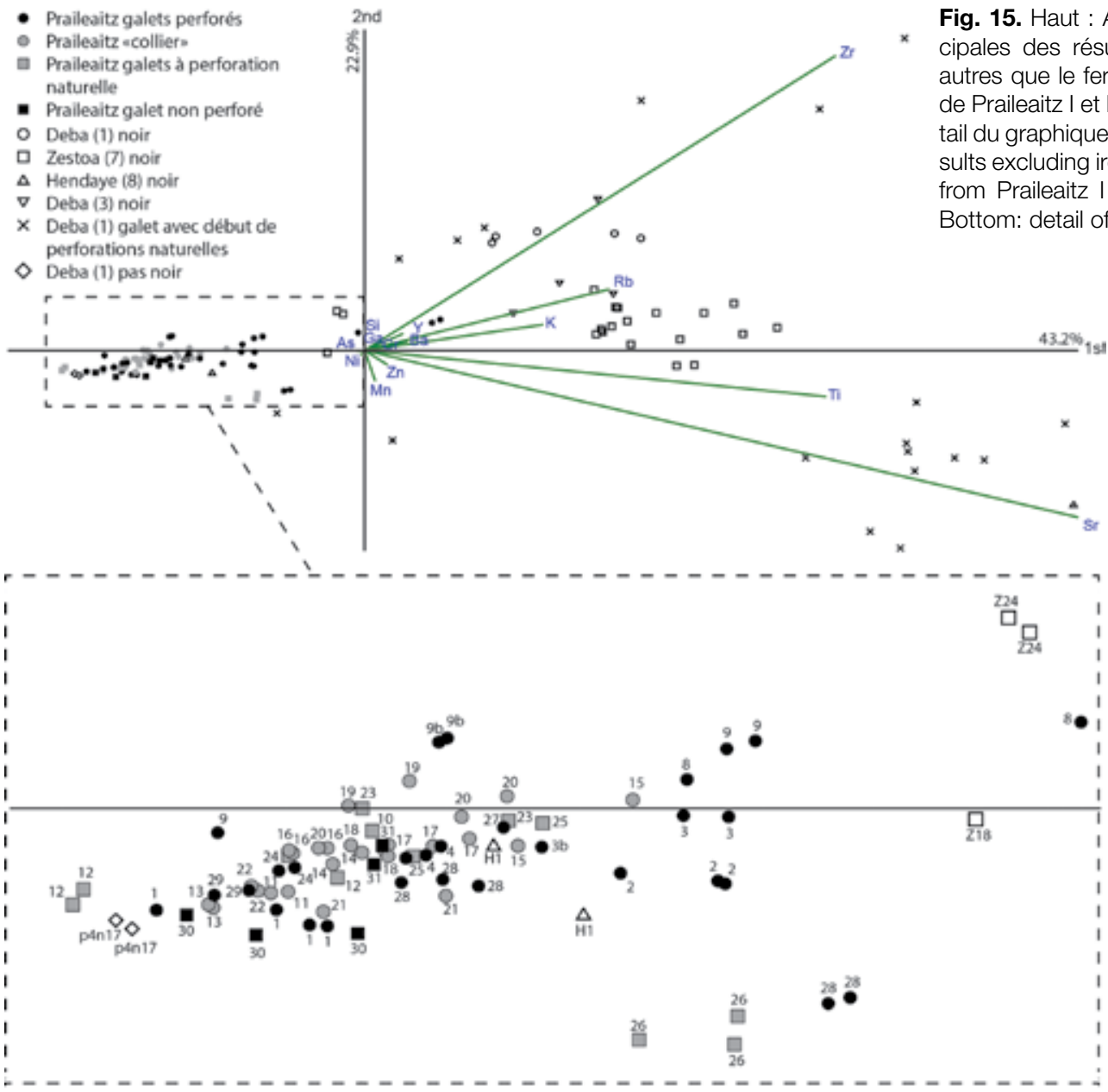


de l'outil perforant. Les autres permettent également de réduire l'épaisseur du galet dans la zone à perforer. Les traces laissées par les techniques de préparation ayant été largement oblitérées par les perforations on ne peut pas exclure, à l'exception de l'abrasion qui laisse des traces très visibles, qu'une technique de préparation non détectée a pu être appliquée, seule ou en combinaison avec d'autres, avant la perforation proprement dite. Cela a pu être le cas pour le seul galet (n. 29) qui ne présente pas de traces de préparation autour de ce qui reste de sa perforation, largement emportée par la fracture de l'extrémité (Fig. 22d). Tous les autres galets avec perforations anthropiques présentent entre une et trois techniques de préparation appliquées à la zone à perforer et cela sur une seule ou sur les deux faces (Tabl. 2). Des variations s'observent entre galets dans la gestuelle avec laquelle les techniques de préparation ont été appliquées. Les incisions sont parallèles, horizontales ou obliques par rapport à l'axe majeur du galet (Tabl. 2). Les traces de râclage et d'abrasion, par contre, sont systématiquement parallèles ou légèrement obliques par rapport à l'axe majeur du galet.

\begin{tabular}{|c|c|c|c|c|c|c|c|c|c|c|c|c|c|c|c|c|}
\hline \multirow[b]{3}{*}{ N. } & \multirow[b]{3}{*}{ Type } & \multicolumn{2}{|c|}{ Dimension } & \multicolumn{2}{|c|}{ Localisation } & \multicolumn{8}{|c|}{ Techn. préparation } & \multicolumn{2}{|c|}{ Orientation I } & \multirow{3}{*}{$\begin{array}{c}\text { Techn. } \\
\text { perforation }\end{array}$} \\
\hline & & \multirow{2}{*}{\begin{tabular}{|l|} 
Dmax \\
$(\mathrm{mm})$ \\
\end{tabular}} & \multirow{2}{*}{$\begin{array}{l}\text { Dmin } \\
(\mathrm{mm})\end{array}$} & \multirow{2}{*}{$\begin{array}{c}\text { Pont Haut } \\
\text { (mm) }\end{array}$} & \multirow{2}{*}{$\begin{array}{c}\text { Orientation } \\
\text { Dmax }\end{array}$} & \multicolumn{4}{|c|}{ Face a } & \multicolumn{4}{|c|}{ Face $b$} & \multirow[t]{2}{*}{ Face a } & \multirow[t]{2}{*}{ Face $b$} & \\
\hline & & & & & & $\mathbf{P}$ & $\mathbf{I}$ & A & $\mathbf{R}$ & $\mathbf{P}$ & I & A & $\mathbf{R}$ & & & \\
\hline 1 & anthropique & 3,77 & 3,66 & 9,13 & perp. & - & $\bullet$ & & & - & $\bullet$ & & & $\mathrm{V}, \mathrm{O}$ & 0 & $\mathrm{RB}$ \\
\hline 2 & anthropique & - & - & - & - & • & & & & • & & & & & & $\mathrm{RB}$ \\
\hline 3 & anthropique & - & - & - & - & & - & & & & & & & V & & $\mathrm{RB}$ \\
\hline 4 & anthropique & 4,22 & 3,89 & 7,51 & perp. & - & - & & & • & - & & & V & 0 & $\mathrm{RB}$ \\
\hline 8 & anthropique & 3,97 & 3,47 & 5,58 & perp. & & - & & & & $\bullet$ & & & V & $\mathrm{V}, \mathrm{O}$ & $\mathrm{RB}$ \\
\hline 9 & anthropique & 3,37 & 3,06 & 7,66 & perp. & & - & & & & - & & & V & $\mathrm{H}$ & $\mathrm{RB}$ \\
\hline 10 & naturelle & 6,44 & 4,43 & $6,68^{\star}$ & - & - & - & - & - & - & - & - & - & - & - & - \\
\hline 11 & anthropique & 3,84 & 3,43 & 2,79 & perp. & $\bullet$ & • & & & 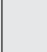 & • & & & $\mathrm{H}, \mathrm{O}$ & $\mathrm{H}, \mathrm{O}$ & $\mathrm{RB}$ \\
\hline 12 & naturelle & 6,64 & 5,95 & $12,03^{*}$ & - & - & - & - & - & - & - & - & - & - & - & - \\
\hline 13 & anthropique & 3,01 & 2,72 & 2,87 & perp. & & - & - & & & . & - & & $\mathrm{V}, \mathrm{O}$ & & $\mathrm{RB}$ \\
\hline 14 & anthropique & 3,05 & 2,48 & 4,05 & perp. & & - & • & & & • & • & & V & $\mathrm{V}, \mathrm{H}$ & $\mathrm{RB}$ \\
\hline 15 & anthropique & 3,96 & 3,67 & 5,35 & par. & & - & & & & & & & V & & $\mathrm{RB}$ \\
\hline 16 & anthropique & 2,74 & 2,28 & na & obl. & & & • & & & • & - & & & V & $\mathrm{RB}$ \\
\hline 17 & anthropique & 3,11 & 2,83 & 4,77 & obl. & - & & & & & & & & & & $\mathrm{RB}$ \\
\hline 18 & anthropique & 3,37 & 3,08 & 4,47 & perp. & - & & & • & & & & - & & & $\mathrm{RB}$ \\
\hline 19 & anthropique & 3,29 & 2,1 & 2,85 & perp. & & - & & & & - & & & V & V & $\mathrm{RB}$ \\
\hline 20 & anthropique & 3,21 & 3 & 5,2 & perp. & $\bullet$ & & & & - & & & & & & $\mathrm{RB}$ \\
\hline 21 & anthropique & 3,02 & 2,78 & 3,55 & par. & & - & & & & - & & & 0 & $\mathrm{~V}, \mathrm{O}$ & $\mathrm{RB}$ \\
\hline 22 & anthropique & 2,82 & 2,55 & 2,63 & par. & & - & - & & - & - & • & & V & $\mathrm{V}, \mathrm{O}$ & $\mathrm{RB}$ \\
\hline 23 & naturelle & 3,93 & 3,61 & $6,83^{*}$ & - & - & - & - & - & - & - & - & - & - & - & - \\
\hline 24 & anthropique & 3,44 & 3,13 & 4,13 & perp. & & • & & & & - & & & V & V & $\mathrm{RB}$ \\
\hline 25 & naturelle & 15,39 & 8,72 & $7,25^{\star}$ & par. & - & - & - & - & - & - & - & - & - & - & - \\
\hline 26 & naturelle & 8,22 & 7,75 & $11,66^{\star}$ & - & - & - & - & - & - & - & - & - & - & - & - \\
\hline 27 & anthropique & - & 3,11 & - & - & & - & & & & • & & & V & $\mathrm{V}, \mathrm{O}$ & $\mathrm{RB}$ \\
\hline 28 & anthropique & - & 2,78 & - & - & $\bullet$ & & & & • & & & & & & $\mathrm{RB}$ \\
\hline 29 & anthropique & - & - & 3,38 & - & & & & & & & & & & & $\mathrm{RB}$ \\
\hline
\end{tabular}

Tabl. 2. Informations concernant les perforations des galets de Praileaitz I. / Information on the perforations of the pebbles from Praileaitz I. 

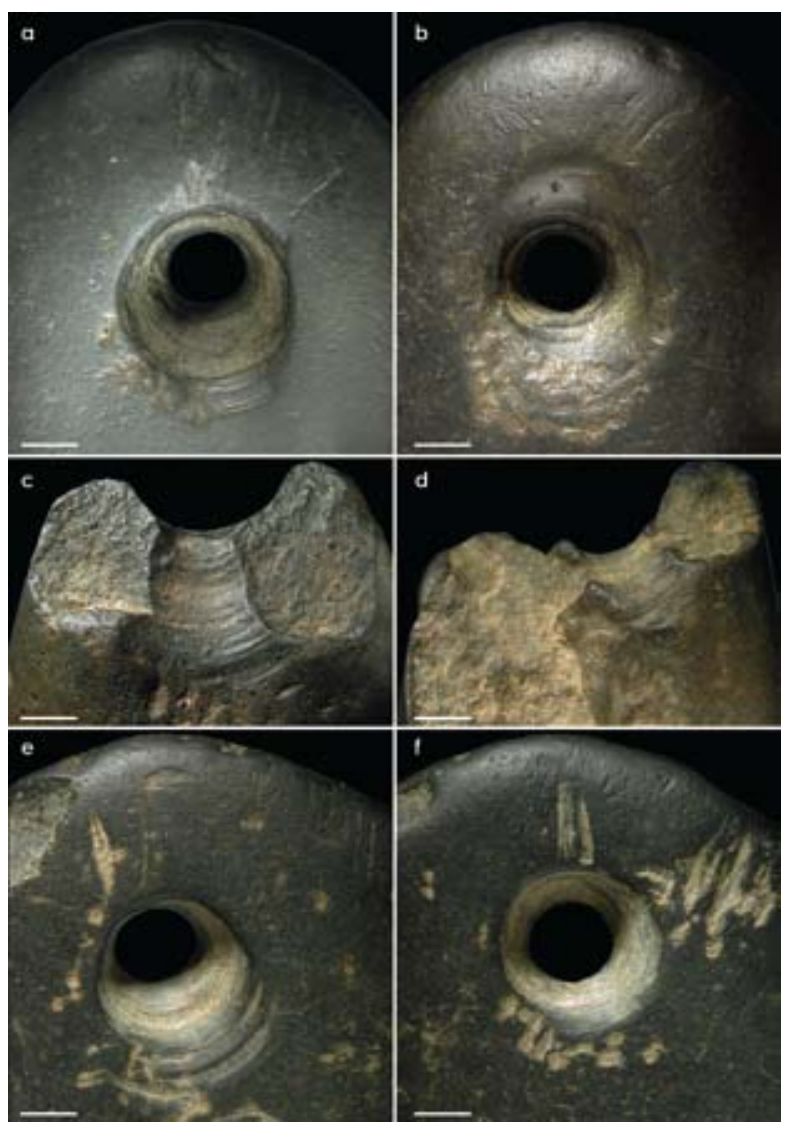

Fig. 16. Macrophotos des perforations sur les galets $n .1(a, b)$ n. 2 (c), n. 3 (d) et n. 4 (e, f) de Praileaitz I. Echelles $=250 \mu$ m. $/$ Macrophotos of perforation on pebbles n. 1 (a, b), n. 2 (c), n. 3 (d) and n. $4(\mathrm{e}, \mathrm{f})$ from Praileaitz I. Scale bars $=250 \mu \mathrm{m}$.

Les perforations ont été réalisées, au moins dans la phase finale du travail, par un outil lithique pointu avec un bord irrégulier, probablement un perçoir retouché à en juger par la profondeur des stries laissées sur les parois du trou. L'analyse des faces opposées de la même perforation (Figs. 16-22) révèle une remarquable homogénéité dans la section de la perforation, l'orientation de son axe et la morphologie des zones striées, indice du fait que la perforation a été élargie en alternance sur les deux faces par la même personne et en appliquant le même geste. Dans la plupart des cas l'artisan s'est attaché à produire des perforations biconiques et évasées, sur les deux faces, vers le centre du galet. Ce procédé présente l'avantage de ne pas réduire la robustesse du pont entre la perforation et l'extrémité du galet. Sur quelques galets par contre, deux parois abruptes opposées côtoient deux parois évasées, indice du fait que l'artisan a exercé un geste tournant à $45^{\circ}$ sans changer sa façon de tenir le galet tout le long du travail. La localisation de ces quatre zones indique qu'il s'agit probablement de droitiers. II est intéressant enfin de remarquer que des différences existent dans les techniques de préparation de la zone à perforer entre les galets du 'collier' et les autres. La préparation par abrasion et par râclage ne se rencontre en effet

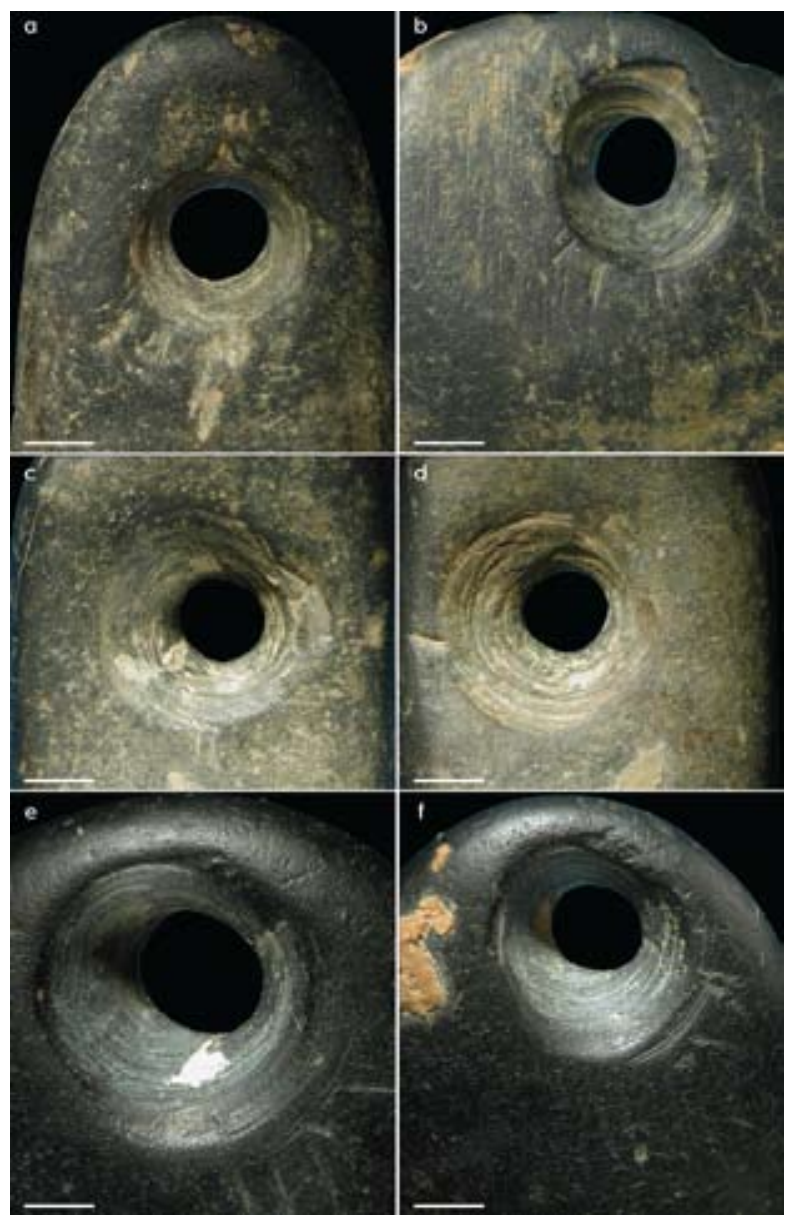

Fig. 17. Macrophotos des perforations sur les galets $n .8$ (a), n. 9 (c-d), n. 11 (e, f) et n. 13 (b) de Praileaitz I. Echelles $=250 \mu \mathrm{m}$. / Macrophotos of perforation on pebbles n. 8 (a), n. 9 (c-d), n. 11 (e, f) and n. 13 (b) from Praileaitz I. Scale bars $=250 \mu \mathrm{m}$.

que parmi les galets appartenant au 'collier'. Cette différence est significative : au contraire d'autres techniques qui laissent des traces qui ont pu être détruites par la perforation, l'abrasion laisse des traces particulièrement étalées et produit une facette d'usure. Si cette technique avait été utilisée sur des pendeloques autres que celles du « collier » elle aurait été facilement détectée. De plus, aucune corrélation ne s'observe entre l'épaisseur du galet et le choix de l'abrasion pour préparer la zone à perforer. L'application de cette technique ne semble pas répondre à des contraintes techniques mais être plutôt liée à des pratiques individuelles ou d'un groupe restreint.

La perforation expérimentale d'un galet collecté dans l'estuaire de la Bidasoa (Fig. 12) et présentant une texture et composition élémentaire proche des galets de Praileaitz I (Figs. n. 14 et 15) a demandé environs cinq heures de travail et l'emploi de cinq perçoirs et quatre burins en différents types de silex. Ces outils ont été régulièrement affûtés au cours du travail jusqu'à leur exhaustion. Nous avons remarqué que pour faire avancer de manière efficace le processus de perforation il était 

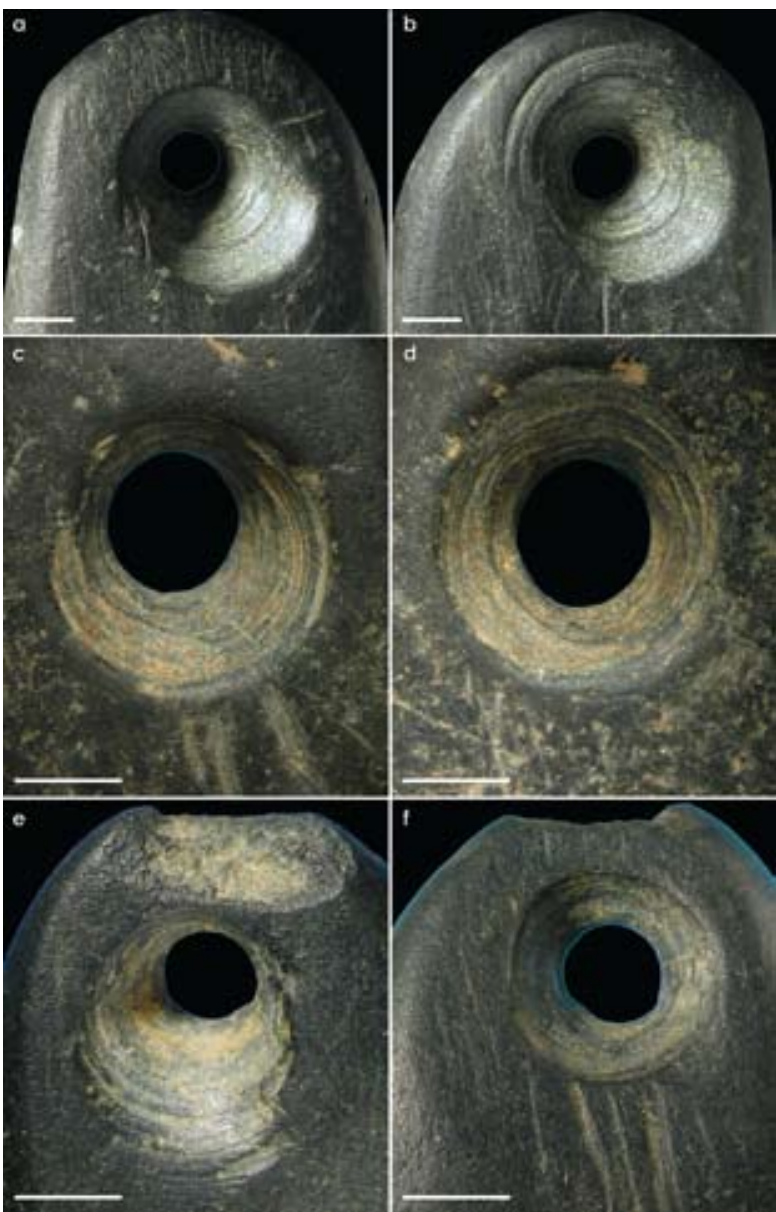

Fig. 18. Macrophotos des perforations sur les galets $n$. 14 (a, b), n. 15 (c, d) et n. 16 (e, f) de Praileaitz I. Echelles $=250 \mu \mathrm{m}$. Macrophotos of perforations on pebbles n. 14 (a, b), n. 15 (c, d) and n. $16(e, f)$ from Praileaitz I. Scale bars $=250 \mu \mathrm{m}$.

pratique d'alterner l'utilisation de plusieurs outils. En considérant l'épaisseur du galet $(5,53 \mathrm{~mm})$ à l'endroit où la perforation expérimentale a été effectuée et la durée de celle-ci on peut considérer qu'un millimètre par heure représente une bonne estimation du rendement d'un artisan averti dans cette tâche. L'épaisseur des galets de Praileaitz I à la hauteur de la perforation varie de $8,7 \mathrm{~mm}$ à $3,5 \mathrm{~mm}$ avec une moyenne de $5,4 \mathrm{~mm}$. La somme de l'épaisseur perforé étant de $112 \mathrm{~mm}$ on peut estimer à une centaine d'heures au minimum le temps nécessaire à la perforation des galets retrouvés dans la grotte.

\subsection{Technique du décor}

Dix-huit des vingt-cing galets avec perforations anthropiques portent un décor (Tabl. 3, Fig. 23). Au contraire, aucun galet présentant une perforation naturelle n'est décoré. Le décor est constitué de traits gravés et d'encoches (Tabl. 3). Les traits gravés ont été réalisés par le passage unique d'une pointe en silex (Figs. 24-26), les

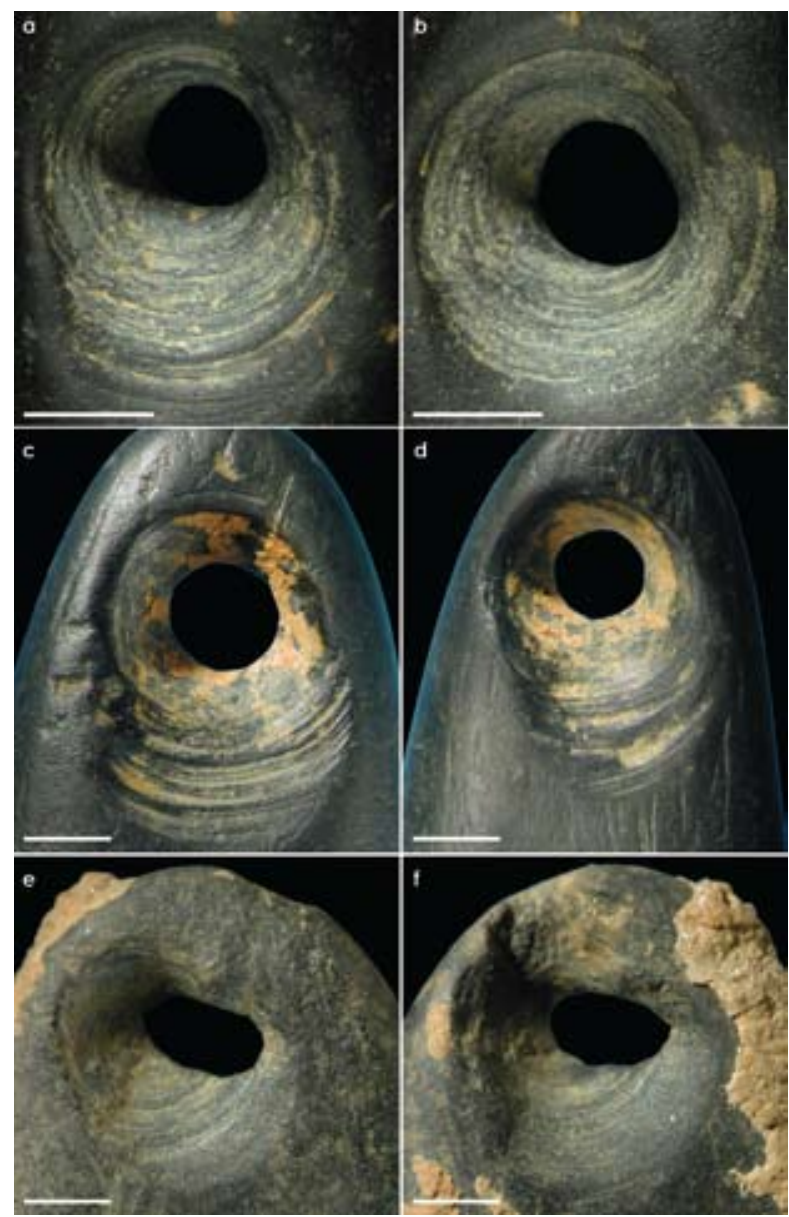

Fig. 19. Macrophotos des perforations sur les galets $n$. 17 (a, b), n. 18 (c, d) et n. 19 (e, f) de Praileaitz I. Echelles = $250 \mu \mathrm{m}$. / Macrophotos of perforations on pebbles n. $17(\mathrm{a}, \mathrm{b}), \mathrm{n} .18(\mathrm{c}, \mathrm{d})$ and n. $19(\mathrm{e}, \mathrm{f})$ from Praileaitz I. Scale bars $=250 \mu \mathrm{m}$.

encoches par le passage unique ou multiple d'un tranchant lithique (Figs. 27-32). Les décors réalisés avec des traits gravés ont été systématiquement tracés sur les faces planes des galets, ceux constitués d'entailles sur les bords. L'analyse microscopique révèle que tous les traits gravés sur un galet sont l'œuvre de la même pointe et du même individu. Les traits ont été réalisés l'un à la suite de l'autre avec des gestes répétés (Figs. 24-26). Ce diagnostic se base sur la morphologie interne du trait et son changement le long de son parcours (Figs. 24a-C, 25a-c, 26a,b), sur des changements dans le point de contact de l'outil au cours de la gravure qui se matérialisent par une suite de traits décalés (Figs. 24d,e), et par la présence d'une strie parasite latérale due au contact momentané avec la surface du galet d'une zone de la pointe (Fig. 25g). Les traits gravés sur les deux faces de la pièce $n .3$ ont des traits de morphologie interne identiques et possèdent les mêmes changements de points de contact (Figs. 24d,e) indiquant ainsi que le même artisan est responsable du décor gravé sur les deux faces de cette pièce. 

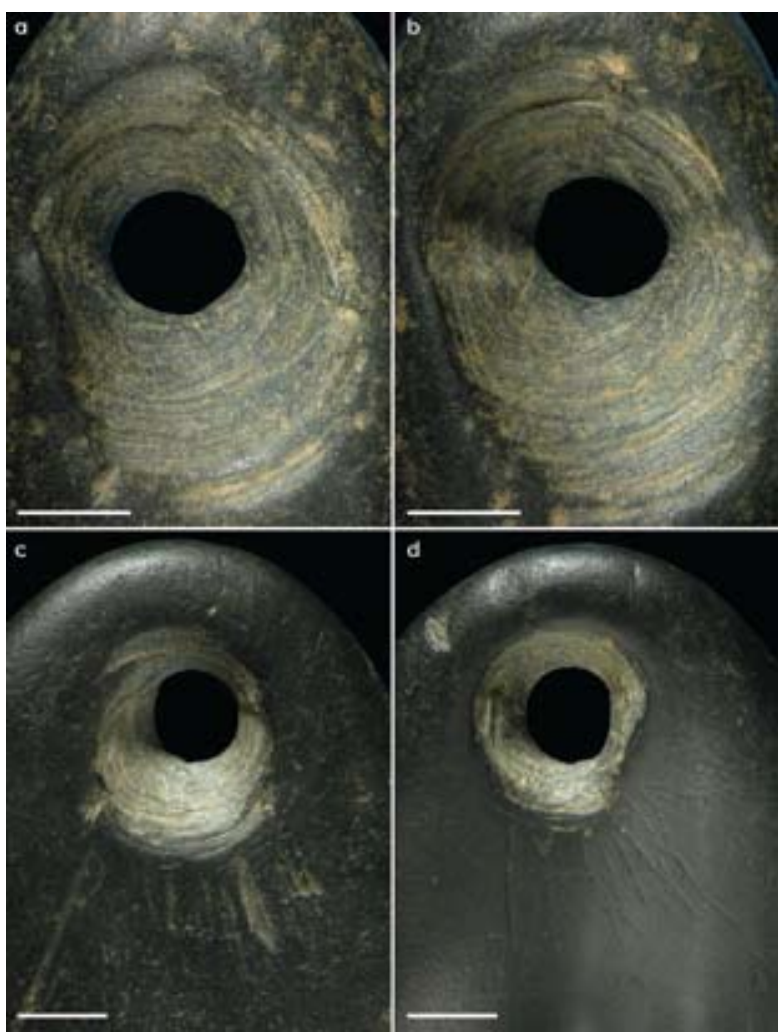

Fig. 20. Macrophotos des perforations sur les galets $n .20(a, b)$ et n. 21 (c, d) de Praileaitz I. Echelles $=250 \mu \mathrm{m}$. / Macrophotos of perforations on pebbles n. 20 (a, b) and n. 21 (c, d) from Praileaitz I. Scale bars $=250 \mu \mathrm{m}$.

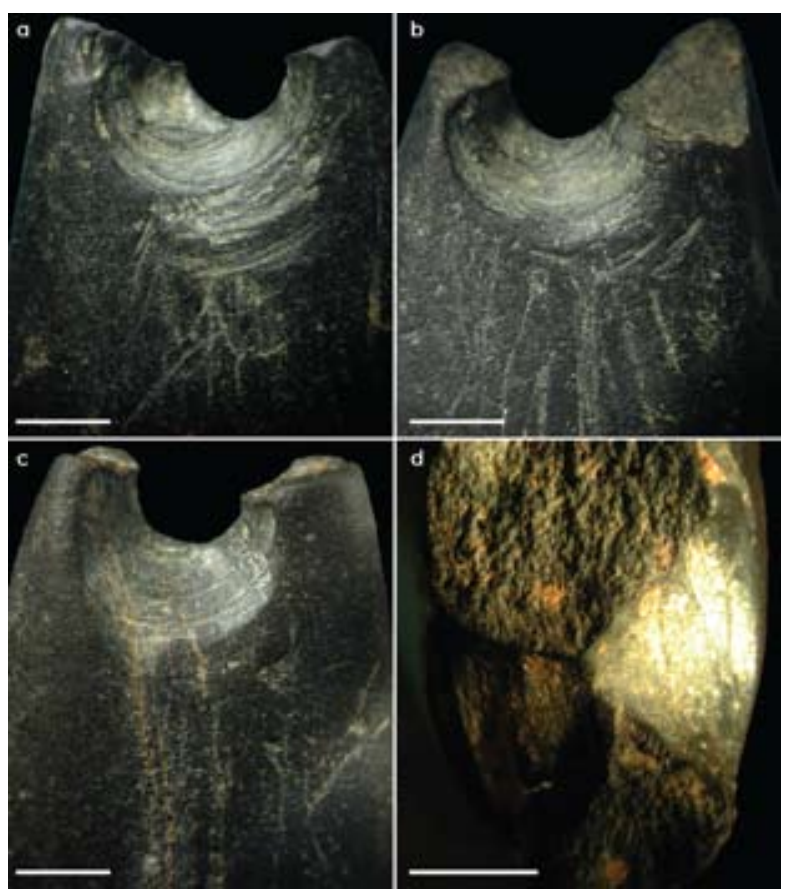

Fig. 22. Macrophotos des perforations sur les galets $n .27$ (a, b), n. 28 (c) et n. 29 (d) de Praileaitz I. Echelles $=250 \mu \mathrm{m}$. / Macrophotos of perforations on pebbles n. 27 (a, b), n. 28 (c) and n. 29 (d) from Praileaitz I. Scale bars $=250 \mu \mathrm{m}$.
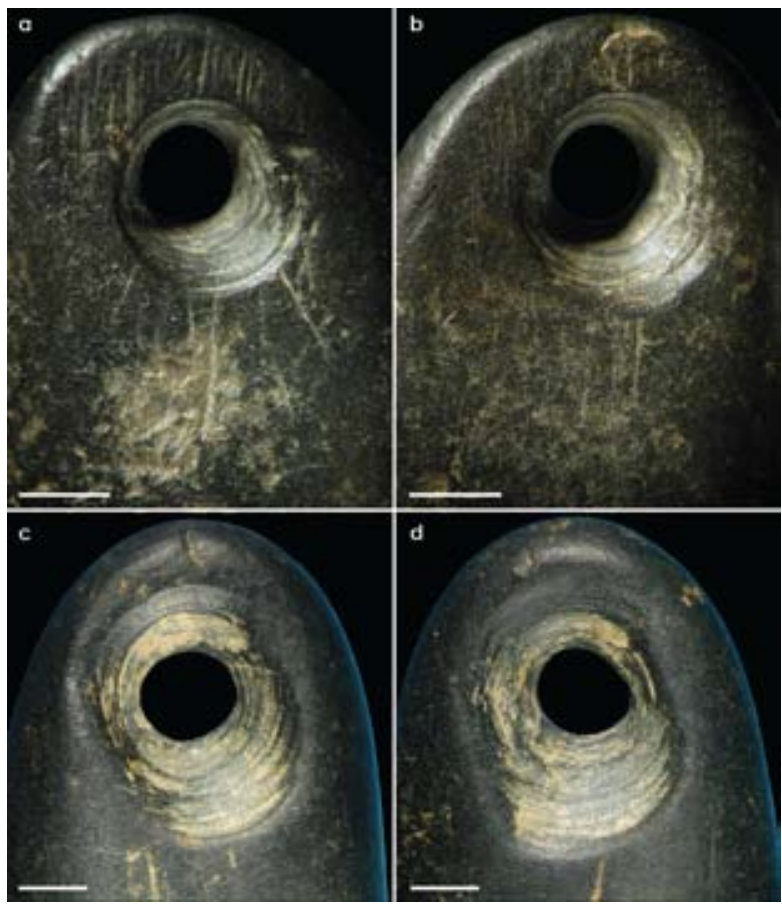

Fig. 21. Macrophotos des perforations sur les galets $n .22(a, b)$ et n. 24 (c, d) de Praileaitz I. Echelles $=250 \mu \mathrm{m}$. / Macrophotos of perforations on pebbles n. $22(a, b)$ and n. 24 (c, d) from Praileaitz I. Scale bars $=250 \mu \mathrm{m}$. 


\begin{tabular}{|c|c|c|c|c|c|c|c|c|c|c|c|}
\hline \multirow[b]{3}{*}{ N. } & \multirow[b]{3}{*}{ Perforation } & \multicolumn{10}{|c|}{ Décor } \\
\hline & & \multicolumn{2}{|c|}{ Face a } & \multicolumn{2}{|c|}{ Face $b$} & \multicolumn{2}{|c|}{ Face c } & \multicolumn{2}{|c|}{ Face d } & \multicolumn{2}{|c|}{ Face e } \\
\hline & & Tec. & $\mathrm{Nb}$ & Tec. & $\mathrm{Nb}$ & Tec. & $\mathrm{Nb}$ & Tec. & $\mathrm{Nb}$ & Tec. & $\mathrm{Nb}$ \\
\hline 1 & anthropique & $\mathrm{T}$ & 6 & & & & & & & & \\
\hline 2 & anthropique & T & & & & E & 12 & E & 12 & E & 17 \\
\hline 3 & anthropique & T & 14 & $\mathrm{~T}$ & 14 & E & 10 & E & 6 & & \\
\hline 4 & anthropique & & na & & & & & & & & \\
\hline 8 & anthropique & & na & & & E & 8 & E & 8 & & \\
\hline 9 & anthropique & & na & & & & & & & & \\
\hline 10 & naturelle & & na & & & & & & & & \\
\hline 11 & anthropique & & na & & & E & 21 & $\mathrm{E}$ & 25 & & \\
\hline 12 & naturelle & & na & & & & & & & & \\
\hline 13 & anthropique & & na & & & E & 12 & E & 17 & & \\
\hline 14 & anthropique & $\mathrm{T}$ & 11 & & & & & $E$ & 23 & & \\
\hline 15 & anthropique & $\mathrm{T}$ & $>23$ & $\mathrm{~T}$ & $>8$ & E & 14 & $E$ & 14 & & \\
\hline 16 & anthropique & & na & & & E & 13 & $E$ & 14 & & \\
\hline 17 & anthropique & T & 13 & & & E & 4 & E & 12 & & \\
\hline 18 & anthropique & $\mathrm{T}$ & 17 & & & E & 11 & E & 4 & & \\
\hline 19 & anthropique & $\mathrm{T}$ & 11 & & & & & E & 9 & & \\
\hline 20 & anthropique & & na & & & E & 11 & $E$ & 9 & & \\
\hline 21 & anthropique & $\mathrm{T}$ & 5 & & & E & 5 & & & & \\
\hline 22 & anthropique & & na & & & E & 15 & & & & \\
\hline 23 & naturelle & & na & & & & & & & & \\
\hline 24 & anthropique & & na & & & E & 4 & E & 17 & & \\
\hline 25 & naturelle & & na & & & & & & & & \\
\hline 26 & naturelle & & na & & & & & & & & \\
\hline 27 & anthropique & & na & & & E & 21 & E & 19 & & \\
\hline 28 & anthropique & & na & & & & & E & 11 & & \\
\hline 29 & anthropique & & na & & & & & & & & \\
\hline & & & & & & & & & & & \\
\hline
\end{tabular}

Tabl. 3. Informations concernant les décors des galets de Praileaitz I. / Information on the decorations of the pebbles from Praileaitz I.

Comme les traits gravés, les séries d'entailles qui décorent les bords des galets ont été réalisées par le même outil et la même main dans une suite de gestes répétés (Figs. 27-32). Sur plusieurs galets on reconnaît la main du même artisan sur les deux bords du même galet (Figs. 27, 28). Ce diagnostic se base sur des critères établis expérimentalement qui permettent, à partir de la morphologie de l'entaille, d'identifier la position de face ventrale et dorsale du tranchant au cours du travail, de savoir si celui-ci était retouché et d'identifier des entailles réalisées par le même outil à partir de la section des entailles et de leur morphologie interne (D'ERRICO, 1998). Des changements dans la morphologie d'entailles successives s'observent sur les galets de Praileaitz I mais ils sont dus à l'usure du tranchant et non à un changement d'outil qui pourrait indiquer une interruption au cours de l'accumulation de ces marques. Les quelques rares cas de changements marqués dans la morphologie d'entailles limitrophes peuvent s'expliquer aisément par le côtoiement d'entailles de début et fin de série (Fig. 27g) ou un changement ou retournement de l'outil à cause d'une usure devenue trop marquée pour poursuivre efficacement le travail. Des différences marquées entre galets s'observent dans le soin que les artisans ont porté à la réalisation et disposition des traits (Figs. 24a-c, 25g) et des entailles (Figs. 27a-g, 30g-i). La reproduction expérimentale de ces marques indique que cette 

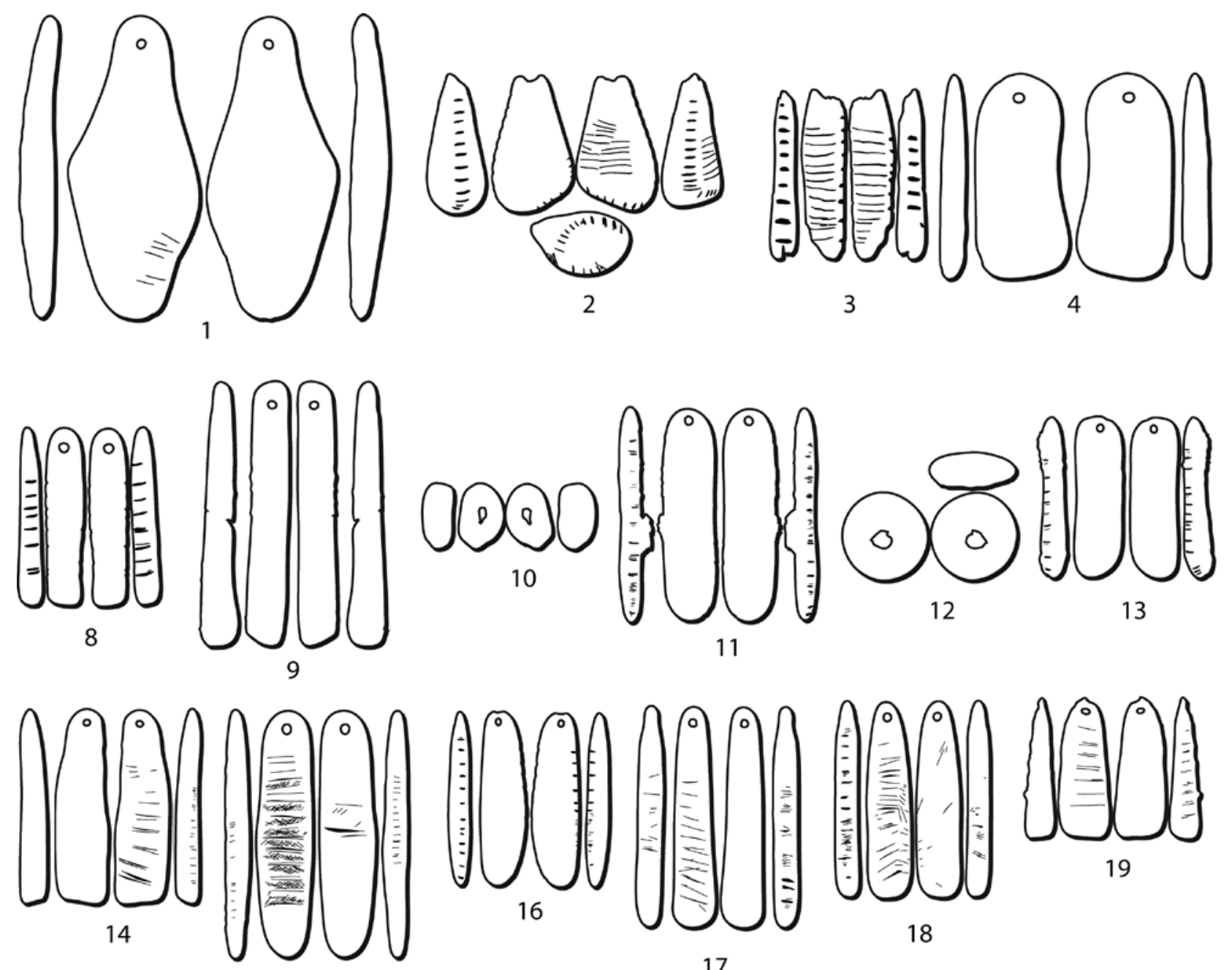

15
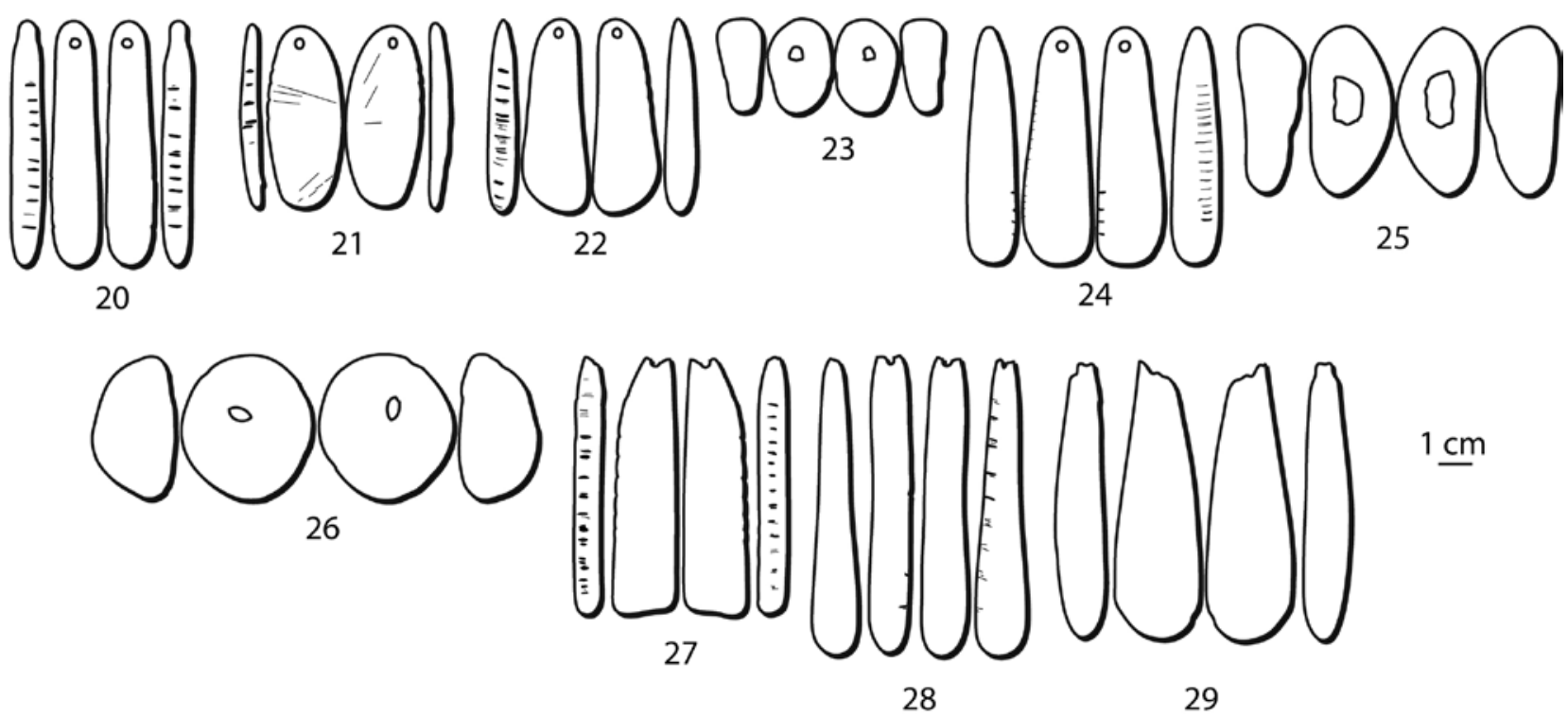

Fig. 23. Relevé des décors sur les galets de Praileaitz I. / Drawings of the Praileaitz I pebbles recording notches and engraved lines 

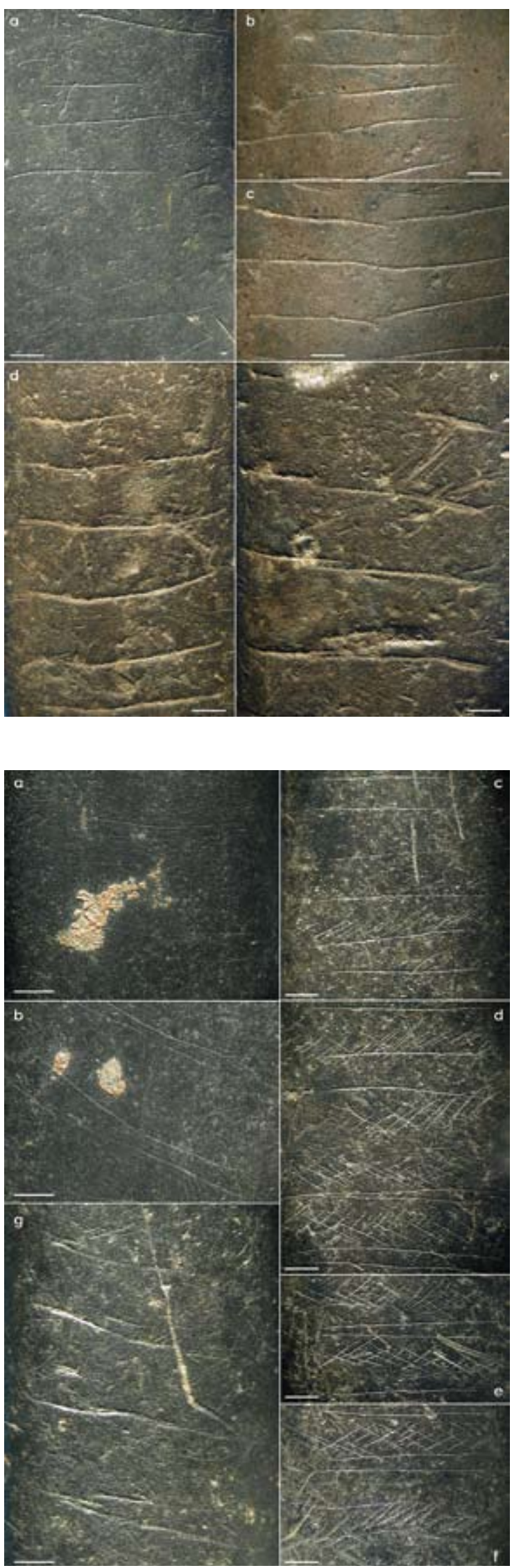

Fig. 24. Macrophotos des traits gravés sur la face ' $a$ ' de la pièce n. 1 (a), la face 'b' de la pièce n. 2 (b, c) et les faces 'a' (d) et 'b' (e)

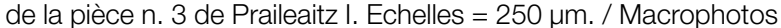
of engraved lines on face 'a' of pebble $n$. 1 (a), face 'b' of pebble n. 2 (b, c) and faces ' $a$ ' (d) and 'b' (e) of pebble n. 3 from Praileaitz I. Scale bars $=250 \mu \mathrm{m}$.

Fig. 25. Macrophotos des traits gravés sur la face ' $b$ ' de la pièce $n$. $14(a, b)$, et sur la face 'a' des pièces n. 15 (c-f) et $\mathrm{n} .17 \mathrm{~g}$ ) de Praileaitz I. Echelles $=250 \mu \mathrm{m}$. / Macrophotos of engraved lines on face ' $b$ ' of pebbles $n .14(a, b)$, face "a" of pebbles n. 15 (c-f) and n. 17 (g) from Praileaitz I. Scale bars $=250$ $\mu \mathrm{m}$
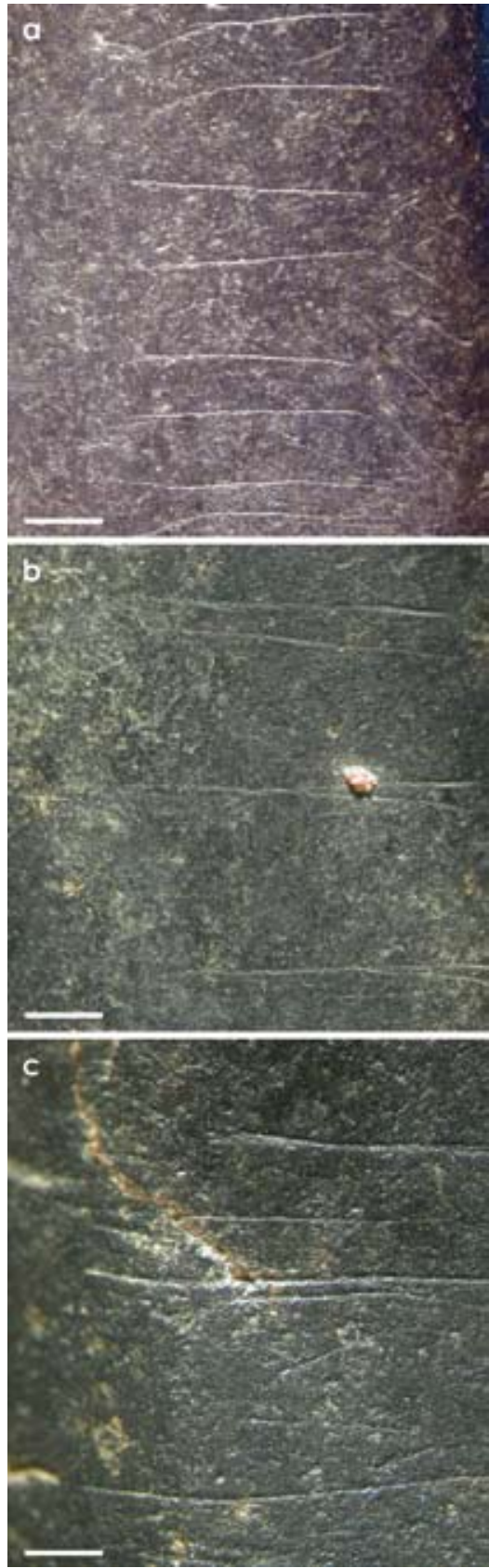

Fig. 26. Macrophotos des traits gravés sur la face 'a' des pièces n. 18 (a), n. 19 (b) et n. 21 (c) de Praileaitz I. Echelles $=250 \mu \mathrm{m}$. / Macrophotos of engraved lines on face ' $a$ ' of pebbles n. 18 (a), n. 19 (b) and n. 21 (c) from Praileaitz I. Scale bars $=250 \mu \mathrm{m}$. 

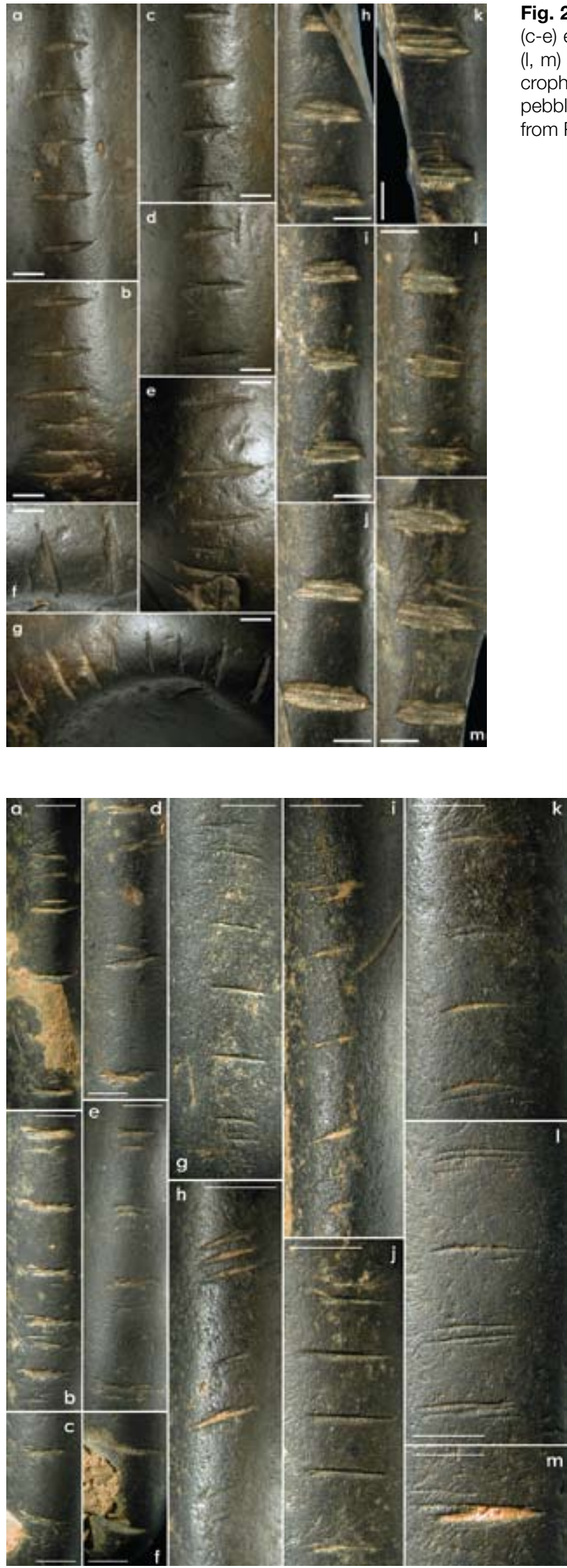

Fig. 27. Macrophotos des encoches sur les faces 'c' (a, b), 'd' (c-e) et 'e' (f, g) de la pièce n. 2 et sur les faces 'd' ( $h, k)$ et 'c' $(\mathrm{l}, \mathrm{m})$ de la pièce n. 3 de Praileaitz I. Echelles $=250 \mu \mathrm{m}$. / Macrophotos of notches on faces 'c' (a,b), 'd' (c-e) and 'e' (f, g) of pebble n. 2 and on faces ' $d$ ' $(h, k)$ and 'c' $(l, m)$ of pebble $n .3$ from Praileaitz I. Scale bars $=250 \mu \mathrm{m}$.

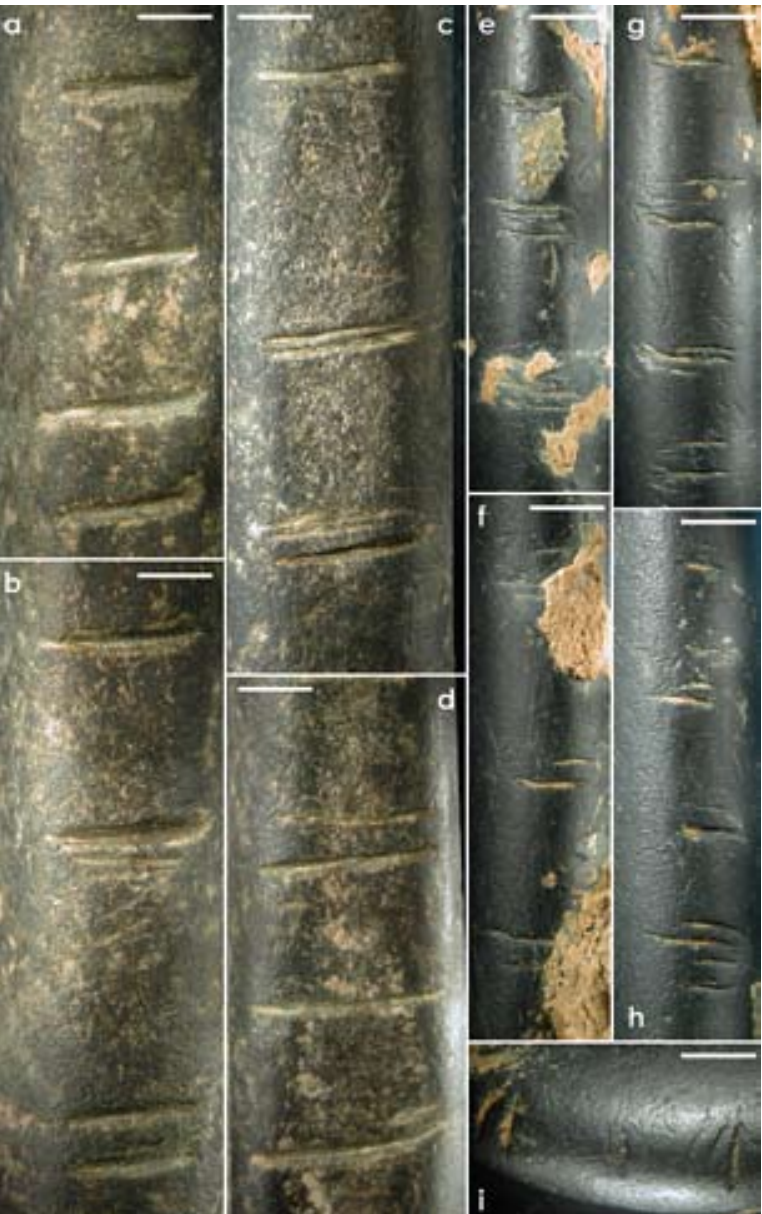

Fig. 28. Macrophotos des encoches sur les faces ' $d$ ' $(a, b)$ et ' $c$ ' $(c, d)$ de la pièce $n .8$ et sur les faces ' $d$ ' (e-g) et ' $c$ ' ( $h$-i) de la pièce n. 11 de Praileaitz I. Echelles $=250 \mu \mathrm{m}$. / Macrophotos of notches on faces ' $d$ ' (a, b) and ' $c$ ' (c, d) of pebble n. 8 and on faces ' $d$ ' $(\mathrm{e}-\mathrm{g})$ and ' $\mathrm{c}$ ' ( $\mathrm{h}-\mathrm{i})$ of pebble n. 11 from Praileaitz I. Scale bars = $250 \mu \mathrm{m}$.

Fig. 29. Macrophotos des encoches sur les faces ' $d$ ' (a-c) et ' $c$ ' $(d-f)$ de la pièce $n .13$, sur la face ' $d$ ' ( $g$ ) de la pièce $n$. 14 et sur les faces ' $d$ ' ( $h$, i) et 'c' (j-m) de la pièce $n .15$ de Praileaitz I. Echelles $=250 \mu \mathrm{m}$. / Macrophotos of notches on faces ' $d$ ' (a-c) and ' $c$ ' (d-f) of pebble n. 13, on face ' $d$ ' (g) of pebble n. 14 and on faces ' $d$ ' $(h, i)$ and ' $c$ ' (j-m) of pebble $n .15$ from Praileaitz I. Scale bars = $250 \mu \mathrm{m}$. 


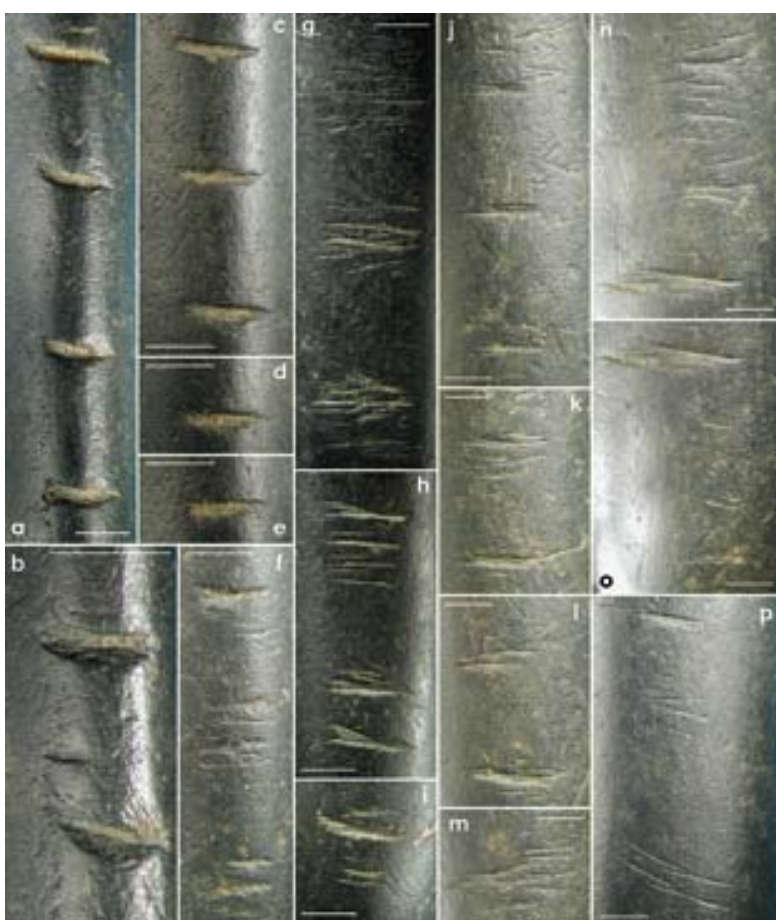

Fig. 30. Macrophotos des encoches sur les faces ' $d$ ' (a, b) et ' $c$ ' (c-f) de la pièce $n$. 16, sur la face ' $c$ ' $(g-i)$ de la pièce $n$. 17 et sur les faces ' $d$ ' (j-n) et ' $c$ ' (o, p) de la pièce $n .18$ de Praileaitz $l$. Echelles $=250 \mu \mathrm{m}$. / Macrophotos of notches on faces ' $d$ ' $(a, b)$ and ' $c$ ' ( $(c-f)$ of pebble $n$. 16, on face ' $c$ ' ( $g$-i) of pebble $n$. 17 and on faces ' $d$ ' ( $j-n)$ and ' $c$ ' $(o, p)$ of pebble n. 18 from Praileaitz I. Scale bars $=250 \mu \mathrm{m}$.

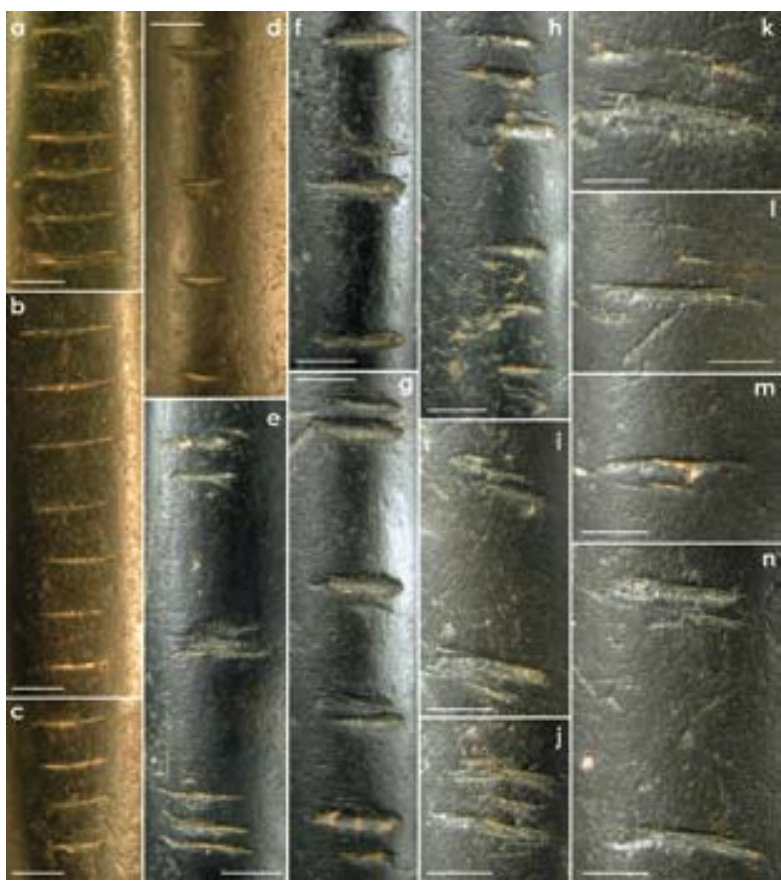

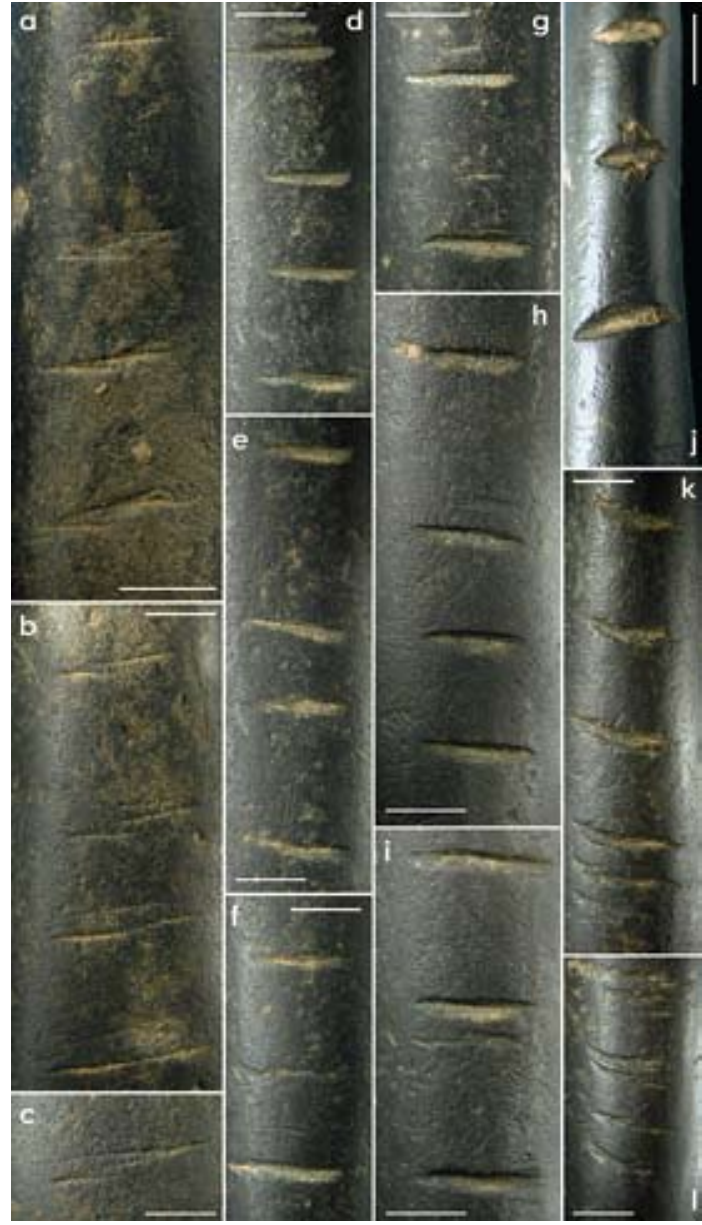

Fig. 31. Macrophotos des encoches sur la face ' $c$ ' (a-c) de la pièce $n .19$, sur les faces ' $d$ ' $(d-f)$ et ' $c$ ' $(g-i)$ de la pièce n. 20, sur la face ' $d$ ' (j) de la pièce $n .21$ et sur la face ' $d$ ' (k, l) de la pièce n. 22 de Praileaitz I. Echelles $=250 \mu \mathrm{m}$. / Macrophotos of notches on face 'c' (a-c) of pebble n.19, on faces ' $d$ ' ( $d-f)$ and ' $c$ ' ( $g$-i) of pebble $n$. 20, on face ' $d$ ' (j) of pebble n. 21 and on face 'd' (k, l) of pebble n. 22 from Praileaitz I. Scale bars $=250 \mu \mathrm{m}$.

Fig. 32. Macrophotos des encoches sur les faces 'c' (a-c) et 'd' (d) de la pièce n. 24, sur la face 'd' (e-h) de la pièce n. 27 et sur la face 'c' (i-n) de la pièce n. 28 de Praileaitz I. Echelles $=250 \mu \mathrm{m}$. / Macrophotos of notches on faces ' $c$ ' (a-c) and ' $d$ ' (d) of pebble $n$. 24, on face ' $d$ ' (e-h) of pebble n. 27 and on face 'c' (i-n) of pebble n. 28 from Praileaitz I. Scale bars $=250 \mu \mathrm{m}$. 
variabilité est due au fait que les décors sont l'œuvre de plusieurs individus ayant des compétences techniques et des niveaux de dextérité différents. En dépits de leur proximité spatiale, les galets du 'collier' se caractérisent par une forte variabilité dans le degré de compétence et d'investissement technique dans la production des entailles. Par contre, les entailles présentes sur les galets découverts dans le vestibule (pièces n. 2, 3 et 8) sont toutes réalisées avec soin et disposées de façon équidistante.

\subsection{Type de décor}

Un seul galet a été décoré exclusivement avec des traits gravés, neuf avec des encoches et huit avec à la fois des traits gravés et des encoches (Tabl. 3, Fig. 23). Parmi les neuf galets qui portent des traits gravés, deux ont été gravés sur les deux faces. Cinq types de décors se distinguent en ordre d'importance sur les galets gravés : a) traits parallèles et équidistants, perpendiculaires à l'axe majeur du galet et couvrant l'ensemble de sa surface (pièces n. 3 et n. 17), b) traits parallèles et équidistants, perpendiculaires à l'axe majeur du galet mais couvrant juste une portion de sa surface (pièces n.1 et n. 21), c) couples juxtaposés de traits parallèles, perpendiculaires à l'axe majeur du galet et couvrant l'ensemble de sa surface (pièces n. 14 et n. 19), d) deux bandes juxtaposées de traits parallèles se rejoignant à une extrémité (pièces n. 2 et n. 18), e) couples de traits parallèles remplis de hachures entrecroisées (pièce n. 15).

\begin{tabular}{|c|c|c|c|c|c|c|}
\hline \multirow[b]{2}{*}{ N. } & \multirow[b]{2}{*}{ Type } & \multicolumn{2}{|c|}{ Perforation } & \multirow[b]{2}{*}{ Décor } & \multicolumn{2}{|c|}{ Surface } \\
\hline & & Degré & Loc. & & Stries & Impacts \\
\hline 1 & galet perforé & intense & $\mathrm{H}$ & & $\bullet$ & $\bullet$ \\
\hline 2 & galet perforé & * & * & & $\bullet$ & $\bullet$ \\
\hline 3 & galet perforé & * & * & & $\bullet$ & \\
\hline 4 & galet perforé & moyen & $\mathrm{H}$ & & $\bullet$ & \\
\hline 8 & galet perforé & moyen & $\mathrm{H}$ & & & \\
\hline 9 & galet perforé & faible & $\mathrm{H}$ & & $\bullet$ & \\
\hline 10 & pierre à $\mathrm{P}$ naturelle & intense & $P$ & & & \\
\hline 11 & galet perforé & intense & $\mathrm{H}, \mathrm{B}$ & & $\bullet$ & \\
\hline 12 & pierre à P naturelle & moyen & $\mathrm{H}, \mathrm{B}$ & & & \\
\hline 13 & galet perforé & moyen & $\mathrm{H}$ & & $\bullet$ & \\
\hline 14 & galet perforé & moyen & $\mathrm{H}$ & $\bullet$ & $\bullet$ & \\
\hline 15 & galet perforé & moyen & $\mathrm{H}$ & $\bullet$ & $\bullet$ & \\
\hline 16 & galet perforé & moyen & $\mathrm{H}$ & & $\bullet$ & \\
\hline 17 & galet perforé & intense & $\mathrm{H}, \mathrm{P}$ & $\bullet$ & $\bullet$ & \\
\hline 18 & galet perforé & intense & $\mathrm{H}, \mathrm{P}$ & & $\bullet$ & $\bullet$ \\
\hline 19 & galet perforé & faible & $P$ & & & \\
\hline 20 & galet perforé & moyen & $\mathrm{H}$ & & $\bullet$ & \\
\hline 21 & galet perforé & moyen & $\mathrm{H}$ & & $\bullet$ & \\
\hline 22 & galet perforé & moyen & $\mathrm{H}$ & & $\bullet$ & $\bullet$ \\
\hline 23 & pierre à P naturelle & intense & $P$ & & & \\
\hline 24 & galet perforé & intense & $\mathrm{H}, \mathrm{P}$ & & $\bullet$ & $\bullet$ \\
\hline 25 & pierre à $\mathrm{P}$ naturelle & intense & $P$ & & $\bullet$ & \\
\hline 26 & pierre à P naturelle & intense & $\mathrm{P}$ & & & \\
\hline 27 & galet perforé & moyen & * & & - & \\
\hline 28 & galet perforé & moyen & * & & $\bullet$ & \\
\hline 29 & galet perforé & intense & * & & $\bullet$ & $\bullet$ \\
\hline
\end{tabular}

Tabl. 4. Informations concernant les traces d'usure et d'utilisation sur les galets de Praileaitz I. / Information on the usewear and utilisation of the pebbles from Praileaitz I. 
Onze galets portent des séries d'entailles sur les deux bords, cinq sur un seul bord (Tabl. 3, Fig. 23). La pièce n. 2, de forme conique aplatie, représente un cas particulier. Des entailles sont gravées sur les deux bords mais également sur le pourtour de sa base. Le nombre d'entailles par bord varie entre 4 et 25 mais il est difficile de les décompter sur les galets où les entailles sont superficielles ou composées de plusieurs traits limitrophes.

\subsection{Traces d'utilisation}

Toutes les perforations portent des usures indiquant que les galets ont été suspendus (Tabl. 4, Figs. 16-22). Ces usures sont plus accusées dans la zone de la perforation proche de l'extrémité du galet, preuve que cette zone était celle qui subissait le frottement le plus intense avec le lien (Figs. 16b, 17e,f). Sur huit galets, I'usure affecte l'ensemble de la perforation et, sur deux autres, seulement la partie la plus proche de l'extrémité du galet. Cela suggère que le lien attachant ces pièces leur laissait un certain degré de mouvement. L'intensité de l'usure de la perforation varie selon les galets, y compris dans celles du 'collier', indice que ce dernier est composé d'objets ayant eu des durées d'utilisation différentes. Les galets avec perforation naturelle présentent des usures intenses autour des perforations qui s'étalent parfois à l'ensemble de la face perforée.

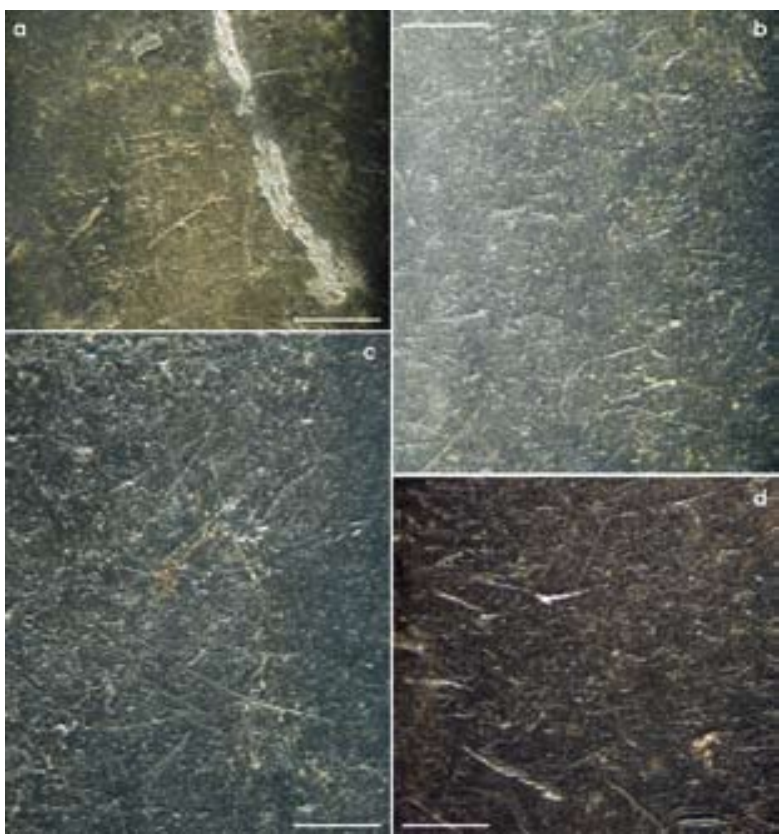

Fig. 33. Stries interprétées comme liées à l'usure sur les pièces $n$. 9 (a), n. 16 (b), n. 27 (c) et n. 22 (d) de Praileaitz I. Echelles = 250 $\mu \mathrm{m}$. / Striations interpreted as traces of usewear on pebbles n. 9 (a), n. 16 (b), n. 27 (c) and n. 22 (d) from Praileaitz I. Scale bars = $250 \mu \mathrm{m}$.

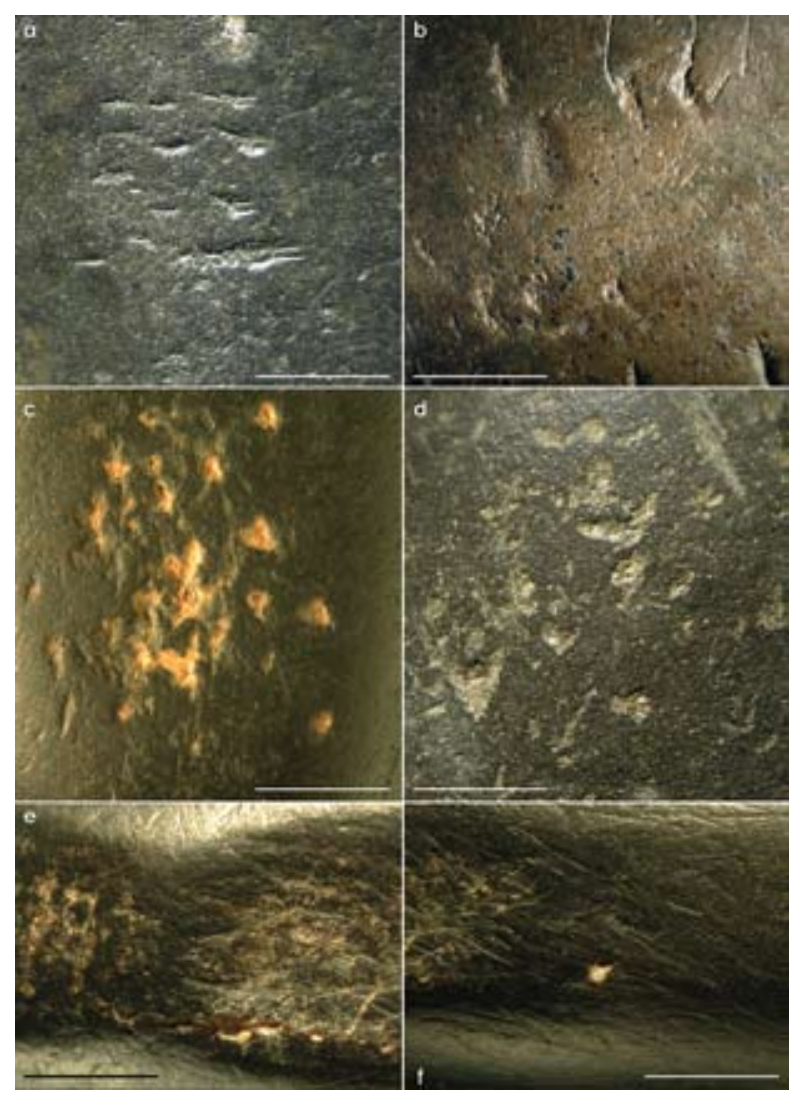

Fig. 34. Traces de percussion sur les pièces n. 1 (a), n. 2 (b), n. 18 (c), n. 29 (e, f) et sur le retouchoir (d) de Praileaitz I. Echelles = 250 um. / Percussion marks on pebbles n. 1 (a), n. 2 (b), n. 18 (c), n. $29(\mathrm{e}, \mathrm{f})$ and on the retoucher (d) from Praileaitz I. Scale bars = $250 \mu \mathrm{m}$

Une usure différentielle affecte aussi les décors qui apparaissent émoussés par endroits sur certains galets (pièces n. 14, 15, 17). Nous interprétons comme usures dues au frottement des galets pendant leur suspension des concentrations de stries localisées sur les faces plates de la plupart des galets (Tabl. 4). Ces concentrations sont constituées de stries d'orientation aléatoire avec une prédominance de stries courtes et perpendiculaires à l'axe majeur de l'objet (Fig. 33). Le fait que ces stries sont absentes sur les deux galets non perforés, constitués de la même matière première et provenant du même horizon culturel étaie fortement cette interprétation.

Six galets perforés et un galet non perforé portent des traces d'une utilisation occasionnelle comme percuteur. La pièce n. 1 présente sur une face plate, dans une zone près de l'extrémité opposée à la perforation, une douzaine d'impacts allongés produits par son utilisation pour retoucher un tranchant en silex (Fig. 34a). La pièce n. 2 porte une dizaine d'impacts superficiels et de forme irrégulière sur un côté (Fig. 34b), résultat d'une utilisation comme percuteur sur une pointe. Quelques traits gravés rentrent dans les impacts, indiquant que le décor du galet est postérieur à l'utilisation de ce dernier comme retouchoir. La pièce $n$. 18 révèle sur sa face plate, près de 


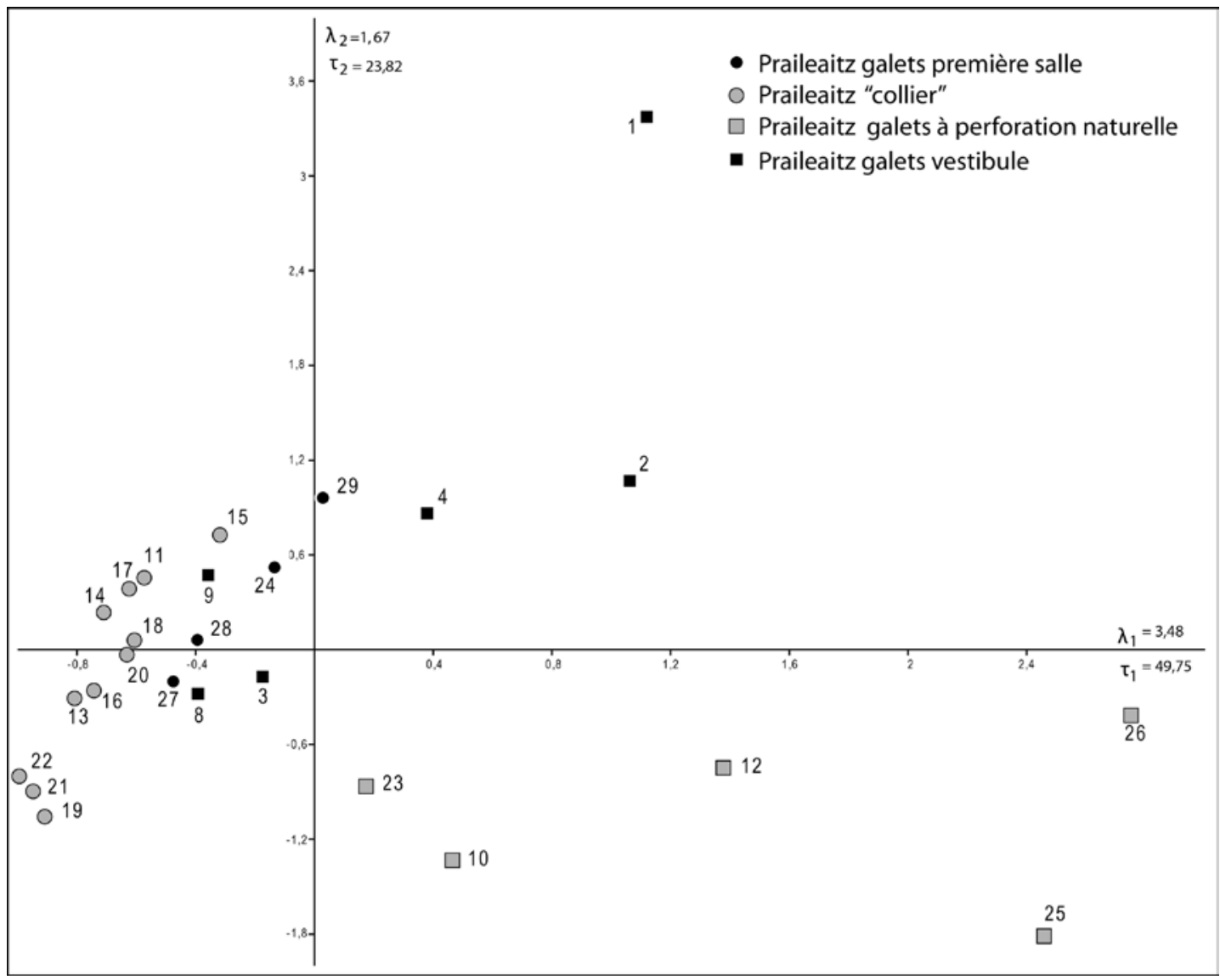

Fig. 35. Analyse en composantes principales des variables liées aux dimensions des galets de Praileaitz I et à la taille et la localisation des perforations. / PCA of variables reflecting the morphometry of the Praileaitz I pebbles and their perforations as well as the location of the perforations.

l'extrémité opposée à la perforation, une trentaine d'impacts ponctiformes dus à son utilisation comme percuteur, probablement pour ré-affûter des burins (Fig. 34c). Des impacts comparables ont été repérés à l'extrémité des galets n. 22 et n. 24. La pièce n. 29 associe, à son extrémité, sur deux zones limitrophes, des impacts semblables à ceux décrits sur les trois pièces précédentes avec de nombreuses stries longitudinales qui effacent partiellement les impacts (Figs. 34e,f). Ces traces sont compatibles avec une utilisation du galet pour retoucher un silex pointu et régulariser un tranchant. Le galet non perforé présente à son extrémité (Fig. 34d) des impacts ponctiformes en tous points semblables à ceux observés sur les galets n. 18, n. 22, n. 24 et n. 29.

\subsection{Le 'collier'}

La figure 35 présente le résultat d'une analyse en composantes principales des variables liées aux dimensions des galets, à la taille et à localisation des perforations. Bien que plus nombreux, les galets appartenant au 'collier' se caractérisent par une plus faible variabilité.
Ceux du vestibule, par contre, révèlent un remarquable éclatement de formes et de types de perforation.

\section{5.- DISCUSSION ET CONCLUSION}

L'approche adoptée dans cette étude permet de reconstituer les choix effectués par les préhistoriques dans la sélection, la modification et l'utilisation des galets de Praileaitz I, d'articuler ces comportements dans l'espace régional et dans l'espace intra-site, de proposer des hypothèses étayées sur la façon dans laquelle les dimensions esthétiques et symboliques de ces objets ont pu retentir sur ces choix.

L'analyse de la composition élémentaire des galets archéologiques et sa comparaison avec la source locale la plus proche et quelques sources éloignées suggère, pour commencer, que le choix du lieu d'approvisionnement et du type de matière première ont fait l'objet de règles strictes. Les galets de Praileaitz I ont été vraisemblablement formés par érosion et transport de fragments de flysch. La forte homogénéité dans la composition élémentaire de ces galets suggère qu'ils proviennent tous, à 
l'origine, du même banc de lutite. L'analyse XRF permet de proposer que les galets archéologiques avec une perforation anthropique n'ont été collectés ni dans la vallée du Deba ni dans celle de l'Urola. L'analyse d'un plus grand nombre de galets noirs transportés par la Bidasoa permettrait de vérifier l'hypothèse que les galets archéologiques ont été ramassés le long de ce cours d'eau ou sur une plage proche de son estuaire, situés à $50 \mathrm{~km}$ du site. Nous ne pouvons cependant pas éliminer pour l'instant, par faute de référentiels adéquats, la possibilité que ces galets aient été collectés dans les vallées d'autres rivières situées à l'est (Oiartzun, Urumea, Oria,) ou à l'ouest du Deba (Artibai, Lea, Mundaka) ou que le banc de flysch constitué par cette lutite ait pu être érodé par plusieurs de ces cours d'eau. Par ailleurs des analyses complémentaires non destructives (Raman, $\mu$ XRD, PIXEPIGE) doivent être envisagées pour mieux caractériser la composition minéralogique et élémentaire des pièces archéologiques et des sources.

Même si, comme démontré par l'analyse morphométrique, des galets proches en couleur et en forme de ceux de Praileaitz I étaient disponibles dans le Deba et l'Urola, les préhistoriques ont préféré sélectionner ailleurs leurs supports. La collecte semble avoir été réalisée à un endroit précis de leur territoire, qui n'est pas dans ces deux cours d'eau. La raison pour cela est sans doute liée à la nature de la matière première : les galets noirs du Deba et de l'Urola ne présentent pas les caractères de dureté et de finesse texturale répondant aux besoins techniques, esthétiques ou symboliques des Magdaléniens de Praileaitz. Des raisons techniques pourraient être liées à la solidité de la perforation et à la pérennité du décor. Les galets noirs locaux sont moins denses que les archéologiques. La perforation expérimentale de ces galets et de celui collecté dans la Bidasoa qui présente une composition proche de celle des galets archéologiques révèle que la perforation des galets locaux est bien plus aisée et rapide mais que la solidité de la perforation est moindre, surtout quand il s'agit de galets plats et ayant un certain poids car allongés. Les préhistoriques ont donc préféré s'obliger à de longues et fastidieuses sessions de travail pour perforer des galets dans une matière première résistante plutôt qu'utiliser des galets de formes proches mais n'ayant pas les mêmes caractéristiques de dureté. Les galets choisis par les préhistoriques se prêtent aussi particulièrement bien à la réalisation de gravures fines qui ne s'effacent pas au cours du temps.

Nos résultats révèlent qu'en plus de la matière première elle-même, le lieu de collecte, resté à l'évidence le même au cours du temps représenté, a pu aussi revêtir une dimension symbolique et devenir un des critères de choix à prendre en compte. Si une provenance éloignée était confirmée par des compléments d'analyse et l'élargissement du référentiel il faudrait aussi envisager la possibilité que les galets aient pu être acquis par échange et que, de ce fait, une partie de leur valeur pour les Magdaléniens dérivait de leur caractère exotique. La composition élémentaire des galets avec perforation naturelle révèle peut être une histoire plus complexe. Certains semblent provenir de la même source éloignée où ont été collectés les galets destinés à être perforés par les préhistoriques, d'autres ont pu être collectés dans le Deba ou dans des sources que nous n'avons pas échantillonées. La raison principale de cette diversification dans les sources d'approvisionnement est probablement à rechercher dans la rareté des supports perforés naturellement. Aucun galet de ce type n'a été trouvé au cours de nos prospections. Cette rareté a poussé les préhistoriques à rechercher systématiquement ces supports au cours de leurs déplacements et déroger dans certains cas sur les critères qui ont déterminé le choix des galets à perforer et à décorer. Ce choix moins regardant se justifie également du point de vue des contraintes liées à la suspension : situées au milieu de galets épais et sub-circulaires, les perforations naturelles ont, au contraire des anthropiques, peu de chances de casser en cours d'utilisation.

Une question centrale à Praileaitz I est celle de savoir pour quelle raison autant de 'pendeloques' en pierre ont été découvertes dans un petit espace et dans des couches qui ont livré un mobilier archéologique relativement peu abondant. Nos analyses montrent que ce site n'est pas un atelier de fabrication d'objets de parure. Les supports ne sont pas locaux et les objets ont été longuement utilisés.

Une bonne partie des perforations, y compris les cassées, ont révélé des traces d'une usure due à la suspension. Les surfaces des galets et les décors portent des usures que l'on ne trouve pas chez les deux spécimens non perforés et qu'il est raisonnable, en considérant leur nature et localisation, d'interpréter comme des usures dues au frottement des galets, au cours de leur suspension, contre des surfaces parsemées d'éléments abrasifs. Les couches archéologiques d'où proviennent les galets n'ont pas livré les dizaines de perçoirs, éclats appointées ou burins qui ont été nécessaires, selon nos résultats expérimentaux, à produire une telle quantité de perforations sur de tels galets. Même si ces outils ont pu être emportés par les préhistoriques après leur utilisation, un certain nombre des pièces avec un fort degré d'exhaustion auraient dû être abandonné dans le site, ce qui n'est pas le cas. Ceci vaut également pour les outils nécessaires à la réalisation des décors gravés ou incisés. L'analyse technologique montre que ces séries de traits et d'encoches ont été faite à la suite et ne peuvent pas de ce fait être interprétées comme des notations basées sur une accumulation de marques au cours du temps. Si l'ensemble des encoches présentes sur les galets avaient été réalisées sur le site nous devrions nous attendre à rencontrer un certain nombre d'outils employés dans cette tâche, ce qui n'est pas le cas.

Ces faits prouvent que les galets ont été apportés sur le site déjà perforés et étayent l'hypothèse que les galets avec des perforations fracturées doivent être interprétés comme des pendeloques cassées accidentellement au cours de la fréquentation du site et perdues ou abandonnées à la suite de la cassure. Les deux spécimens qui ne sont pas perforés, même si constitués de la même matière première, ne se conforment pas aux ca- 
nons dimensionnels identifiés par l'analyse morphométrique des galets perforés volontairement ou naturellement. Leur épaisseur, en particulier, rendrait extrêmement longue une perforation à l'outil lithique. Ces objets apportent la preuve que les préhistoriques ont également exploité la source d'approvisionnement des galets destinés à la production de pendeloques pour des pièces qui n'étaient pas collectées à cette fin et qui pouvaient, à l'occasion, être utilisés comme percuteurs. Une utilisation éphémère pour retoucher ou régulariser les bords d'éclats et des lames en silex ou ré-affûter des burins a été constatée aussi sur des pièces transformées en pendeloque. Le seul cas où une chronologie relative entre ces traces et le décor gravé a pu être établie nous a indiqué que la gravure était plus récente que l'utilisation comme percuteur. Cela ne préjuge pas du fait que l'opposé a pu être vrai pour les autres galets avec de telles traces. La présence de ces traces sur des galets perforés et gravés n'est pas facile à expliquer. Elle semble indiquer en tout cas que la dimension symbolique est précédée ou peut même coexister avec une utilisation fonctionnelle.

Le constat que les galets ont été apportés au site déjà perforés ne nous éclaire pas pour autant sur les raisons qui ont déterminé leur perte ou abandon dans cette petite grotte. II est probable que ces raisons et les circonstances d'abandon soient différentes selon les concentrations d'objets et les deux zones de fréquentation principales. Les galets du 'collier' se caractérisent, en plus de leur proximité spatiale, par une plus faible variabilité dans leur morphologie et dans la taille et la localisation des perforations. Deux techniques de préparation de la zone où est ensuite pratiquée la perforation, l'abrasion et le râclage, ne s'observent que sur les galets du 'collier'. Ces galets participent par contre de la même variabilité observée sur l'ensemble des galets du site pour ce qui concerne le degré d'usure des perforations, les techniques de décors, et les motifs gravés et incisés. Cette situation est compatible avec l'interprétation du collier comme un ensemble d'objets produits à des moments différents et par des artisans différents, partageant néanmoins certaines techniques et des critères de choix. Les différents degrés d'usure observés sur les perforations suggèrent que les galets du collier ont pu intégrer plusieurs autres dispositifs de parure avant d'être assemblés dans celui abandonné à Praileaitz.

L'ensemble de pendeloques appartenant au 'collier' donnerait donc l'image de la variabilité de ces pendeloques quand elles étaient intégrées dans un seul dispositif de parure, utilisées par un individu ou par un groupe restreint. Proposer une hypothèse sur la nature et fonction de ce dispositif est un exercice forcément spéculatif.

Ces pendeloques pouvaient être intégrées dans un long collier porté par un individu, cousues sur un vêtement, une couverture ou sur un sac de couchage, attachées à une corde traversant l'espace d'habitat ou cousues sur un rideau partageant entièrement ou partiellement cet espace. Cette liste de possibilités n'est sans doute pas exhaustive. Toute hypothèse doit néanmoins être compatible avec la localisation et l'orientation des galets au moment de leur découverte ainsi qu'avec le constat d'un décalage dans la longueur des pendeloques du centre vers les extrémités de leur distribution spatiale. Pour que cette symétrie puisse être reconnue visuellement, les pendeloques, ou la majorité d'entre elles, devaient pouvoir, au moins dans certaines circonstances, être perçues par un observateur. Cela est difficilement concevable dans le cas d'un collier, si on accepte que certaines pendeloques étaient situées, comme on

Fig. 36. Dessin schématique d'une cape décorée de pendeloques. / Drawing of a cape decorated with elongated stone pendants.
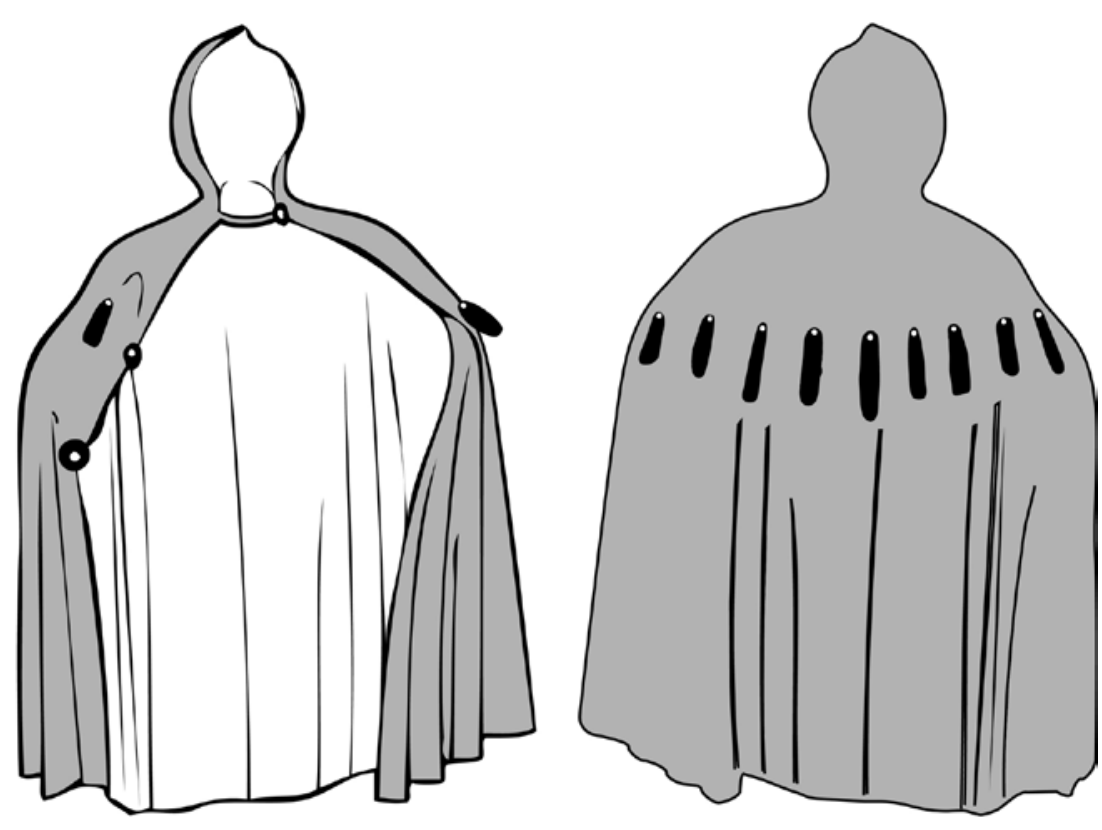
l'observe sur le plan de la fouille, jusqu'à $20 \mathrm{~cm}$ l'une de l'autre et que dans son ensemble le dispositif auquel les pendeloques étaient fixées s'étalait sur une longueur de $120 \mathrm{~cm}$ à $150 \mathrm{~cm}$. Pour ces mêmes raisons il est improbable que les pendeloques aient pu être accrochées à un rideau. Un rideau d'une telle largeur permettrait une bonne vision des pendeloques mais il serait trop court pour être utilisé efficacement dans ce site. Pour toutes ces raisons l'hypothèse qui nous semble la plus probable est celle d'une utilisation de ces pendeloques pour décorer une couverture ou une cape en fourrure pouvant jouer à l'occasion aussi le rôle de couverture (Fig. 36). Cette explication est compatible avec la disposition des galets sur le site et offre une explication pour la présence de galets à perforation naturelle aux extrémités de la distribution. Ils pourraient s'agir de boutons servant à attacher la cape. L'hypothèse d'une cape pourrait être étayée dans le futur par une reconstitution expérimentale d'un tel vêtement.

Quoi qu'il en soit, la proximité spatiale des galets du 'collier', leur disposition et la découverte de deux galets avec des perforations en contact, indices d'un faible déplacement post-dépositionnel, plaide pour considérer que l'abandon du 'collier' correspond au dernier ou à un des derniers épisodes de fréquentation de cette salle par les Magdaléniens.

Tout en partageant un certain air de famille avec ceux du 'collier', les autres galets découverts dans la même salle pourraient avoir été abandonnés dans cet espace au cours de fréquentations bien antérieures. Ces galets peuvent d'ailleurs être partagés dans deux lots distincts et pas nécessairement contemporains. Deux galets à perforation naturelle (n. 25 et 26) et un galet présentant une perforation anthropique non fracturée (n. 24) ont été exhumés au centre de la salle, à proximité du foyer découvert dans cette zone. Trois autres galets (n. 27, 28, 29), tous avec la perforation fracturée, et un fragment de galet à perforation fracturée (n. 3) ont été collectés contre la paroi opposée à celle où a été trouvé le 'collier'. La découverte, dans la même zone, de plusieurs galets avec perforation fracturée peut difficilement être due au hasard et reflète probablement une utilisation répétée de cette zone de la salle dans une activité distincte de celle qui a conduit à l'abandon du 'collier' ou des objets près du foyer central. Pour résumer, les galets de la salle peuvent être interprétés comme le reflet d'une utilisation répété dans le temps et par un même groupe du même espace pour y mener des activités qui ont amené à la fracture et à l'abandon des pendeloques.

Les galets découverts dans le vestibule se distinguent de ceux de la salle par l'absence de pièces à perforation naturelle et par une variabilité morphologique accusée. Ils sont en effet soit plus effilés soit plus larges que ceux trouvés dans la salle et incluent des types uniques (rhomboïde, cône). À bien voir et exception faite pour la pièce n. 3, la seule qui, comme par hasard, remonte avec un fragment découvert dans la salle, aucun des galets découverts dans le vestibule n'est assimilable à ceux découverts dans la salle. La forte variabilité mor- phologique des galets du vestibule peut être raisonnablement attribuée au fait que ces derniers étaient à l'origine intégrés dans des dispositifs de parure différents. Ils pourraient représenter le résultat de pertes survenues au cours de fréquentations plus étalées dans le temps et dues à des membres de plusieurs groupes magdaléniens, qui auraient de préférence occupé la zone proche de l'entrée de la cavité. Selon ce scénario les galets du vestibule nous donnent une image de la variabilité d'utilisation de ces pendeloques à l'échelle d'une population plus large ou d'une période plus longue et légèrement décalée par rapport à celle de la salle. Dans un cas comme dans l'autre le facteur de continuité est constitué par la forte propension pour la même matière première et la même source d'approvisionnement.

\section{6.- REMERCIEMENTS}

Nous remercions Xabier Peñalver, Jose Antonio Mujika, Sonia San Jose et Solange Rigaud pour la relecture critique de ce manuscrit ainsi que Arnaud Lenoble, Genna Antonin et Philippe Razin pour les discussions sur la nature et les possibles sources des galets. Sonia San Jose a participé a la création de la collection de référence utilisée dans cette étude. Michel Lenoir a aimablement fourni les outils lithiques utilisés lors de l'expérimentation. Cette recherche a été financée par le projet TRACSYMBOLS (FP7/2007/2013/ERC Grant n.249587).

\section{7.- BIBLIOGRAPHIE}

ÁLVAREZ-FERNANDEZ, E.

2006 Los objetos de adorno-colgantes del Paleolitico superior y del Mesolitico en la Cornisa Cantabrica y en el Valle del Ebro: una vision europea. Universidad de Salamanca. Salamanca.

BLUMENSCHINE, J., MAREAN, C., CAPALDO, S.D.

1996 Blind tests of interanalyst correspondence and accuracy in the identification of cut marks, percussion marks, and carnivore tooth marks on bone surfaces. Journal of Archaeological Science 2, 493-507.

\section{BONNARDIN, S.}

2009 La parure funéraire au Néolithique ancien dans les Bassins parisiens et rhénans - Rubané, Hinkelstein et VilleneuveSaint-Germain. Société Préhistorique Française. Paris. (Mémoires de la Société préhistorique française; 49).

CONARD, N.

2003 Eiszeitlicher Schmuck auf der Schwäbischen Alb, en KÖLB, S., CONARD, N.J. (Eds.). Eiszeitschmuck: Status and Schönheit. 15-49. Urgeschichtliches Museum Blaubeuren. Blaubeuren.

CONKEY, M.

1980 The identification of prehistoric hunter-gatherer aggregation sites: The case of Altamira. Current Anthropology 21, 609-630. 
DAVIDSON, I.

1997 The Power of Pictures, en CONKEY, M., SOFFER, O. (Eds.). Beyond Art. Pleistocene Image and Symbol. 125 159. California Academy of Sciences. San Francisco. (Memoirs of the California Academy of Science; 23, Wattis Symposium Series in Anthropology)

D'ERRICO, F.

1993 La vie sociale de l'art mobilier paléolithique. Manipulation, transport, suspension des objets en os, bois de cervidés, ivoire. Oxford Journal of Archaeology 12(2), 145-174.

1998 Palaeolithic origins of artificial memory systems: an evolutionary perspective, en RENFREW, C., SCARRE, C. (Eds.). Cognition and Material Culture: the Archaeology of Symbolic Storage. 19-50. McDonald Institute. Cambridge (McDonald Institute Monographs).

D'ERRICO, F., VANHAEREN, M.

1999 Les méthodes d'analyse de l'art mobilier paléolithique. Quelques exemples issus de la région cantabrique. Anthropologie et préhistoire 110, 31-45.

2002 Criteria for identifying red deer age and sex from their canines. Application to Upper Palaeolithic and Mesolithic ornaments. Journal of Archaeological Science 29, 211-232.

FRITZ, C.

1999 La gravure dans l'art mobilier magdalénien. Du geste à la représentation. DAF. Paris. (Documents d'archéologie française ; 75).

GROENEN, M

1996 Dépôts et cachettes : permanence et valeur dans la préhistoire paléolithique, en GROENEN, M. (Ed.). La préhistoire au quotidien : mélanges offerts à Pierre Bonenfant. 143-200. Million. Paris.

GURIOLI, F.

2002 Analisi dei reperti in materia dura animale provenienti dal deposito aurignaziano della Grotta di Fumane (VR): Strumenti, Ogetti ornamentali e Superfici con tracce di interventi antropici. Universita di Ferrara. Ferrara.

HENSHILWOOD, C., D'ERRICO, F.

2005 Being Modem in the Middle Stone Age: individuals and innovation, en GAMBLE, C., POOR, M. (Eds.). Individuals in the Paleolithic. 244-264. Routledge University Press. London.

PEÑALVER IRIBARREN, X., MUJIKA ALUSTIZA, J.A.

2003 Suelo de ocupación magdaleniense en la cueva de Praile Aitz I (Deba, Gipuzkoa): evidencias de arte mobiliar. Veleia 20, 157-81.

PEÑALVER IRIBARREN, X., SAN JOSE SANTAMARTA, S.

2008 La grotte de Praileaitz I (Deba, Gipuzkoa, Euskal Herria Pays Basque) et la défense du patrimoine dans le Pays Basque. Préhistoire du Sud-Ouest 16, 143-50.
PEÑALVER, X., SAN JOSE, S., MUJIKA-ALUSTIZA, J.A.

2017 La cueva de Praileaitz I (Deba, Gipuzkoa, Euskal Herria) Intervención arqueológica 2000 - 2009. Munibe Monographs. Anthropology and Archaeology Series, 1.

PEÑALVER, X., SAN JOSE, S., MUJIKA, J.A., OTERO, X.

2006 Praileaitz I Haitzuloko (Deba) Madeleine Aldiko Zintzilikarioak. Gipuzkoako Foru Aldundia (Bertan 22).

RIGAUD, S.

2011 La parure : traceur de la géographie culturelle et des dynamiques de peuplement au passage Mésolithique-Néolithique en Europe. Université Bordeaux 1. Talence.

SOLE, V.A., PAPILLON, E., COTTE, M., WALTER, PH., SUSINI, J.

2007 A multiplatform code for the analysis of energy-dispersive X-ray fluorescence spectra. Spectrochim. Acta Part B 62 63-68.

STINER, M.C.

1999 Palaeolithic mollusc exploitation at Riparo Mochi (Balz Rossi, Italy): food and ornaments from the Aurignacian through Epigravettian. Antiquity 73, 735-754.

TABORIN, Y

1993 La parure en coquillage au Paléolithique. CNRS. Paris. (Gallia Préhistoire ; supl. XXIX).

2004 Langage sans parole : la parure au temps préhistoriques. La Maison Des Roches. Arles.

VANHAEREN, $M$

2006 La parure: de sa production à la projection de l'image de soi, en BODU, P., JULIEN, M., VALENTIN, B., DEBOUT, G. (Eds.). Les Magdaléniens du niveau IVO de Pincevent. 35-49. (Gallia préhistoire ; 48).

2010 Les fonctions de la parure au Paléolithique supérieur : de l'individu à l'unité culturelle. Editions Universitaires Européennes. Sarrebruck.

VANHAEREN, M., D'ERRICO, F.

2003a Le mobilier funéraire de la Dame de Saint-Germain-laRivière et l'origine paléolithique des inégalités sociales. Paléo 15, 195-238.

2003b The Body Ornaments Associated with the Burial, en ZIL HAO, J., TRINKKAUS, E. (Eds.), Portrait of the Artist as a Child. The Gravettian human skeleton from the Abrigo do Lagar Velho and its archaeological context. Instituto Portugues de Archeologia. Lisboa, 154-186. (Trabalhos de Arqueologia, 22).

WHITE, R

1992 Beyond Art: Toward an Understanding of the Origins of Material Representation in Europe. Annual Review of Anthropology 21, 537-564. 